Electronic Journal of Statistics

Vol. 14 (2020) 3429-3486

ISSN: $1935-7524$

https://doi.org/10.1214/20-EJS1743

\title{
High dimensional classification for spatially dependent data with application to neuroimaging
}

\author{
Yingjie Li \\ Department of Statistics and Probability, Michigan State University, East Lansing, \\ MI 48824, USA \\ e-mail: liyingjienk@gmail.com \\ Liangliang Zhang \\ Department of Biostatistics, University of Texas MD Anderson Cancer Center, Houston, \\ TX 77030, USA \\ e-mail: liangliangzhang.stat@gmail.com \\ Tapabrata Maiti* \\ Department of Statistics and Probability, Michigan State University, East Lansing, \\ $M I$ 48824, USA \\ e-mail: maiti@msu.edu
}

\begin{abstract}
Discriminating patients with Alzheimer's disease (AD) from healthy subjects is a crucial task in the research of Alzheimer's disease. The task can be potentially achieved by linear discriminant analysis (LDA), which is one of the most classical and popular classification techniques. However, the classification problem becomes challenging for LDA because of the high-dimensionality and the spatial dependency of the brain imaging data. To address the challenges, researchers have proposed various ways to generalize LDA into high-dimensional context in recent years. However, these existing methods did not reach any consensus on how to incorporate spatially dependent structure. In light of the current needs and limitations, we propose a new classification method, named as Penalized Maximum Likelihood Estimation LDA (PMLE-LDA). The proposed method uses Matérn covariance function to describe the spatial correlation of brain regions. Additionally, PMLE is designed to model the sparsity of highdimensional features. The spatial location information is used to address the singularity of the covariance. Tapering technique is introduced to reduce computational burden. We show in theory that the proposed method can not only provide consistent results of parameter estimation and feature selection, but also generate an asymptotically optimal classifier driven by high dimensional data with specific spatially dependent structure. Finally, the method is validated through simulations and an application into ADNI data for classifying Alzheimer's patients.
\end{abstract}

MSC2020 subject classifications: Primary 62H30; secondary $62 \mathrm{H} 35$. Keywords and phrases: classification, high dimensional, linear discriminant analysis, misclassification, neuroimaging, spatially dependent, tapered covariance.

Received February 2019

${ }^{*}$ Corresponding author. 


\section{Contents}

1 Introduction . . . . . . . . . . . . . . . . . . . . 3430

2 Classification using maximum likelihood estimate (MLE-LDA) . . . . 3435

2.1 Spatial models . . . . . . . . . . . . . . . . 3435

2.2 Classification using MLE-LDA _ . . . . . . . . . . . 3437

3 Classification using penalized maximum likelihood estimate (PMLE-

LDA ) . . . . . . . . . . . . . . . . . . . . . 3440

3.1 The penalized maximum likelihood estimation (PMLE) . . . . 3440

3.1.1 Consistency of one-step PMLE . . . . . . . . . . . . . 3443

3.1.2 Covariance tapering and one-step PMLE . . . . . . . . . . 3444

3.2 The penalized maximum likelihood estimation LDA (PMLE-LDA)

classifier . . . . . . . . . . . . . . . . . . 3445

4 Simulation study . . . . . . . . . . . . . . . . . . . . . . 3447

5 Real data application . . . . . . . . . . . . . . . . . . . . 3450

6 Conclusion and discussion . . . . . . . . . . . . . . . . . . . . 3452

7 Remarks on the assumptions . . . . . . . . . . . . . . . . . . 3454

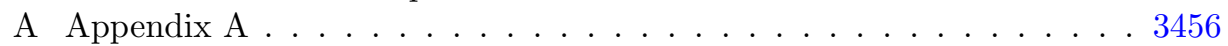

A.1 Proofs for classification using MLE . . . . . . . . . . . 3456

A.2 Proofs for consistency of one-step PMLE . . . . . . . . . 3468

A.3 Proofs for consistency for one-step PMLE with tappering . . . . 3475

A.4 Proofs for classification using PMLE-LDA . . . . . . . . . . 3478

Acknowledgements . . . . . . . . . . . . . . . . . . . . . 3483

References . . . . . . . . . . . . . . . . . . . . . . 3483

\section{Introduction}

This paper is motivated by discriminating patients with Alzheimer's disease (AD) from healthy subjects using structural Magnetic Resonance Imaging (sMRI) data. We also would like to identify the key sMRI features that differentiate the two groups. We translate the real needs as a technical problem of using classification method and selecting features. However, the problem becomes challenging because of the complexity of the data. First, brain imaging data is spatially dependent, which means that the dependence between various voxels (pixels) can be depicted by their proximity. Second, brain imaging data is high-dimensional, because a single subject can produce hundreds of 3D MRI scans and a single 3D MRI scan can generate millions of voxels.

Many existing methods could handle the challenges of brain imaging data, but they did not solve a classification problem. In brain research, the assignment of functional regions has been mainly based on certain assumptions and conceptualizations. In particular, conceptualization of spatial partition and correspondence is widely used in brain imaging analysis (see Worsley [43], Smith and Fahrmeir [39], Lindquist [29], Musgrove, Hughes and Eberly [33], Bowman [6]). These methods exploited spatial dependence of brain imaging data, but they were not designed to classify and identify spatial features. In addition to brain 
imaging, spatial analysis has been broadly applied across agriculture, geology, soil science, oceanography, forestry, meteorology and climatology. Traditionally, these applications are not necessarily high-dimensional. But an increasing trend of big data shows that high-dimensional data with spatially dependent structure attracts more and more interest. For recent developments of regularized models dealing with spatially dependent data, please refer to Hoeting et al. [21], Huang and Chen [23], Zhu, Huang and Reyes [47], Chu et al. [12], Reyes, Zhu and Aukema [36], Fu et al. [20], Nandy, Lim and Maiti [34], Feng et al. [18]. One can simply adapt these available procedures, but again they did not consider how to classify and identify spatial features.

Many high-dimensional approaches are not preferable due to the concern of high variance and overfitting issues (Friedman, Hastie and Tibshirani [19]). So it is necessary to incorporate regularization techniques into the classification method for high dimensional data. Fisher's linear discriminant analysis (LDA) is one of the most classical and popular classification techniques. The simplicity and flexibility of LDA has allowed itself to be extended to many complex and high dimensional applications. Researchers have proposed many ways to generalize LDA into high-dimensional context. However, these existing methods did not reach any consensus on how to incorporate spatial dependence structure. In light of the current needs and limitations, we propose a new LDA procedure accommodating both the complex dependent structure and high-dimensionality.

Before introducing the proposed procedure, we first give a review of the existing LDA methods for high dimensional data. Let us consider the $p$-dimensional discriminant problem between two classes $\mathcal{C}_{1}$ and $\mathcal{C}_{2}$. According to some classification rule $T(\boldsymbol{\Omega}): R^{p} \rightarrow\{1,2\}$, a new observation $\boldsymbol{\Omega}$ can be classified into class $\mathcal{C}_{1}=\{\boldsymbol{\Omega}: T(\boldsymbol{\Omega})=1\}$ or $\mathcal{C}_{2}=\{\boldsymbol{\Omega}: T(\boldsymbol{\Omega})=2\}$. Given that $\boldsymbol{\Omega} \in \mathcal{C}_{1}$, the misclassification rate is the conditional probability of that $\boldsymbol{\Omega}$ is classified into class $\mathcal{C}_{2}$, i.e. $P\left(T(\boldsymbol{\Omega})=2 \mid \boldsymbol{\Omega} \in \mathcal{C}_{1}\right)$. Similarly, $P\left(T(\boldsymbol{\Omega})=1 \mid \boldsymbol{\Omega} \in \mathcal{C}_{2}\right)$ denotes the misclassification rate when $\boldsymbol{\Omega} \in \mathcal{C}_{2}$.

The optimal classifier obtained by minimizing the posterior probability is known as the Bayes rule, which classifies the new observation into the most probable class (Chapter 2 in [19]). Suppose that $f_{k}(\boldsymbol{\omega})$ denotes the density of the misclassification rate that an observation $\boldsymbol{\omega}$ is classified into $\mathcal{C}_{k},(k=1,2)$. Let $\pi_{k}$ be the prior probability of class $k$ with $\pi_{1}+\pi_{2}=1$. According to Bayes theorem, the posterior probability of an observation $\Omega=\boldsymbol{\omega}$ in each class is $P\left(\boldsymbol{\Omega} \in \mathcal{C}_{k} \mid \boldsymbol{\Omega}=\boldsymbol{\omega}\right)=\frac{f_{k}(\boldsymbol{\omega}) \pi_{k}}{f_{1}(\boldsymbol{\omega}) \pi_{1}+f_{2}(\boldsymbol{\omega})\left(1-\pi_{1}\right)}$.

A typical way of modeling the class densities is that they are assumed to be multivariate Gaussian $N\left(\boldsymbol{\mu}_{1}, \Sigma\right)$ and $N\left(\boldsymbol{\mu}_{2}, \Sigma\right)$ respectively, where $\boldsymbol{\mu}_{k}(k=1,2)$ are the class mean vectors and $\Sigma$ is the common positive definite covariance matrix. Then the density of an observation $\Omega=\boldsymbol{\omega}$ from $\mathcal{C}_{k}$ can be written as $f_{k}(\boldsymbol{\omega})=\frac{1}{(2 \pi)^{p / 2}}|\Sigma|^{1 / 2} e^{\left.-\frac{1}{2}\left(\boldsymbol{\omega}-\boldsymbol{\mu}_{k}\right)^{T} \Sigma^{-1}\left(\boldsymbol{\omega}-\boldsymbol{\mu}_{k}\right)\right)}$. Under this assumption, the Bayes rule assigns $\boldsymbol{\Omega}=\boldsymbol{\omega}$ into $\mathcal{C}_{1}$ if $\pi_{1} f_{1}(\boldsymbol{\omega}) \geq \pi_{2} f_{2}(\boldsymbol{\omega})$. Equivalently, $\boldsymbol{\omega}$ is assigned to $\mathcal{C}_{1}$ if $\log \frac{\pi_{1}}{\pi_{2}}+(\boldsymbol{\omega}-\boldsymbol{\mu})^{T} \Sigma^{-1}\left(\boldsymbol{\mu}_{1}-\boldsymbol{\mu}_{2}\right) \geq 0$, where $\boldsymbol{\mu}=\left(\boldsymbol{\mu}_{1}+\boldsymbol{\mu}_{2}\right) / 2$. Notice that this classifier is linear in $\boldsymbol{\omega}$. 
In practice, the parameters of the Gaussian distribution should be estimated using the training data. Suppose that $\boldsymbol{Y}_{k 1}, \ldots, \boldsymbol{Y}_{k n_{k}}$ are training data from class $\mathcal{C}_{k}$, where $k \in\{1,2\}$ and $\boldsymbol{Y}_{k j} \in \mathbb{R}^{p}$ are independent and identically distributed as p dimensional Gaussian distribution $N\left(\boldsymbol{\mu}_{k}, \Sigma\left(\boldsymbol{\theta}_{0}\right)\right)$, where $\boldsymbol{\mu}_{k}=\left(\mu_{k 1}, \ldots, \mu_{k p}\right)^{T}$, $n_{k}$ is the sample size for class $\mathcal{C}_{k} . \Sigma(\boldsymbol{\theta})$ is the covariance matrix with parameter $\boldsymbol{\theta}=\boldsymbol{\theta}_{0}$. Assume that $\hat{\boldsymbol{\mu}}_{1}, \hat{\boldsymbol{\mu}}_{2}, \hat{\Sigma}$ and $\hat{\boldsymbol{\Delta}}$ (generated from $\boldsymbol{Y}_{k 1}, \ldots, \boldsymbol{Y}_{k n_{k}}$ ) are estimates of $\boldsymbol{\mu}_{1}, \boldsymbol{\mu}_{2}, \Sigma$ and $\boldsymbol{\Delta}$, where $\boldsymbol{\Delta}=\left(\Delta_{1}, \ldots, \Delta_{p}\right)^{T}=\boldsymbol{\mu}_{1}-\boldsymbol{\mu}_{2}$ is the difference of the two classes in mean.

Let $n=n_{1}+n_{2}$ be the total sample size. Assume that $\frac{n_{1}}{n} \rightarrow \pi, 0<\pi<1$ as $n \rightarrow \infty$. $p$ depends on $n$. Assume that the two classes have equal prior probabilities, i.e. both the probabilities that a new observation comes from $\mathcal{C}_{1}$ and $\mathcal{C}_{2}$ are $\frac{1}{2}$. Then we obtain the classification rule $\hat{\delta}$ :

$$
\hat{\delta}(\boldsymbol{\Omega})=\left(\boldsymbol{\Omega}-\frac{\hat{\boldsymbol{\mu}}_{1}+\hat{\boldsymbol{\mu}}_{2}}{2}\right)^{T} \hat{\Sigma}^{-1} \hat{\boldsymbol{\Delta}}
$$

A new observation $\boldsymbol{\omega}$ is classified into class $\mathcal{C}_{1}$ if $\hat{\delta}(\boldsymbol{\omega})>0$ and $\mathcal{C}_{2}$ otherwise. If $\boldsymbol{\Omega}$ comes from $\mathcal{C}_{1}$, then the conditional misclassification rate of $\hat{\delta}$ is

$$
W_{1}(\hat{\delta})=P\left(\hat{\delta}(\boldsymbol{\Omega}) \leq 0 \mid \boldsymbol{\Omega} \in \mathcal{C}_{1}, \boldsymbol{Y}_{k i}, i=1,2, \ldots, n_{k}, k=1,2\right)=1-\Phi\left(\Psi_{1}\right)
$$

where

$$
\Psi_{1}=\frac{\left(\boldsymbol{\mu}_{1}-\hat{\boldsymbol{\mu}}\right)^{T} \hat{\Sigma}\left(\hat{\boldsymbol{\mu}}_{1}-\hat{\boldsymbol{\mu}}_{2}\right)}{\sqrt{\left(\hat{\boldsymbol{\mu}}_{1}-\hat{\boldsymbol{\mu}}_{2}\right)^{T} \hat{\Sigma}^{-1} \Sigma \hat{\Sigma}^{-1}\left(\hat{\boldsymbol{\mu}}_{1}-\hat{\boldsymbol{\mu}}_{2}\right)}} .
$$

Similarly, we can define the error rate for observations from $\mathcal{C}_{2}$. If $\boldsymbol{\Omega}$ comes from class $\mathcal{C}_{2}$, the conditional misclassification rate of $\hat{\delta}$ is:

$$
W_{2}(\hat{\delta})=\mathbb{P}\left(\hat{\delta}(\boldsymbol{\Omega})>0 \mid \boldsymbol{\Omega} \in \mathcal{C}_{2}, \boldsymbol{Y}_{k i}, k=1,2 ; i=1, \ldots, n_{k}\right)=\Phi\left(\Psi_{2}\right),
$$

where

$$
\Psi_{2}=\frac{\left(\boldsymbol{\mu}_{2}-\hat{\boldsymbol{\mu}}\right)^{T} \hat{\Sigma}\left(\hat{\boldsymbol{\mu}}_{1}-\hat{\boldsymbol{\mu}}_{2}\right)}{\sqrt{\left(\hat{\boldsymbol{\mu}}_{1}-\hat{\boldsymbol{\mu}}_{2}\right)^{T} \hat{\Sigma}^{-1} \Sigma \hat{\Sigma}^{-1}\left(\hat{\boldsymbol{\mu}}_{1}-\hat{\boldsymbol{\mu}}_{2}\right)}}
$$

As we assume the equal prior probability for the two classes, the overall misclassification rate is defined as

$$
W(\hat{\delta})=\frac{1}{2}\left(W_{1}(\hat{\delta})+W_{2}(\hat{\delta})\right)
$$

If $\boldsymbol{\mu}_{1}, \boldsymbol{\mu}_{2}$ and $\Sigma$ are known, the optimal classification rule is Bayes rule, which classifies a new observation $\boldsymbol{\Omega}=\boldsymbol{\omega}$ into class $\mathcal{C}_{1}$ if

$$
\delta(\boldsymbol{\omega})=\left(\boldsymbol{\omega}-\frac{\boldsymbol{\mu}_{1}+\boldsymbol{\mu}_{2}}{2}\right)^{T} \Sigma^{-1} \boldsymbol{\Delta}>0 .
$$


Bayes rule has the smallest misclassification rate. If $\boldsymbol{\Omega}$ is from class $\mathcal{C}_{1}$ and thus has a normal distribution $N\left(\boldsymbol{\mu}_{1}, \Sigma(\boldsymbol{\theta})\right)$, we can calculate that the conditional misclassification rate of Bayes rule $\delta$ is

$$
W_{1}(\delta)=W_{2}(\delta)=1-\Phi\left(\frac{\sqrt{C_{p}}}{2}\right),
$$

where $C_{p}=\boldsymbol{\Delta}^{T} \Sigma^{-1}(\boldsymbol{\theta}) \boldsymbol{\Delta}$ and $\Phi(\cdot)$ is the standard Gaussian distribution function.

We obtain the overall misclassification rate of Bayes rule as $W(\delta)=1-$ $\Phi\left(\frac{\sqrt{C_{p}}}{2}\right)$. Since Bayes rule has the smallest misclassification rate, we write $W_{O P T}=1-\Phi\left(\frac{\sqrt{C_{p}}}{2}\right)$ as the optimal misclassification rate. Under certain conditions, we could have $C_{p} \rightarrow C_{0}$, then $W_{O P T} \rightarrow 1-\Phi\left(\frac{\sqrt{C_{0}}}{2}\right)$, where $C_{0}$ is a constant.

Using training data, we can estimate the parameters with the sample mean and covariance

$$
\begin{aligned}
& \hat{\boldsymbol{\mu}}_{k}=\sum_{i=1}^{n_{k}} \boldsymbol{Y}_{k i} / n_{k}=\overline{\boldsymbol{Y}}_{k}, \\
& \hat{\Sigma}=\sum_{k} \sum_{i}\left(\boldsymbol{Y}_{k i}-\hat{\boldsymbol{\mu}}_{k}\right)^{T}\left(\boldsymbol{Y}_{k i}-\hat{\boldsymbol{\mu}}_{k}\right) /\left(n_{1}+n_{2}-2\right), k=1,2 .
\end{aligned}
$$

Then LDA classifies $\boldsymbol{\Omega}$ into class $\mathcal{C}_{1}$ if

$$
\hat{\delta}_{L D A}(\boldsymbol{\Omega})=\left(\boldsymbol{\Omega}-\frac{1}{2}\left(\overline{\boldsymbol{Y}}_{1 .}+\overline{\boldsymbol{Y}}_{2} \cdot\right)\right)^{T} \hat{\Sigma}^{-1}\left(\overline{\boldsymbol{Y}}_{1 .}-\overline{\boldsymbol{Y}}_{2}\right)>0
$$

LDA is an asymptotically optimal classifier under traditional large sample scenario, that is, the dimension of variables $(p)$ is fixed and the sample size $(n)$ tends to infinity. However, this is not true in the high dimensional context. Bickel and Levina [3] demonstrated that LDA asymptotically did not perform better than random guessing if $p / n \rightarrow \infty$.

The asymptotic theory of LDA does not hold under high dimensional setting because of two reasons. First, the sample covariance matrix $\hat{\Sigma}$ is singular. It is difficult to estimate the precision matrix $\Omega=\Sigma^{-1}$. To resolve this, the independence rule (IR) ignores the correlations among features and use diagonal of $\hat{\Sigma}$ to replace $\hat{\Sigma}$. Bickel and Levina [3] showed in theory that IR leads to a better classification result than the naive LDA, where the Moore-Penrose inverse is used to replace $\hat{\Sigma}^{-1}$. Another similar way to resolve this issue is the nearest shrunken centroid classifier (Tibshirani et al. [40]). Fan and Fan [15] proposed the feature annealed independence rule (FAIR) that performs feature selection by t-test in addition to IR. The above methods made LDA applicable for high dimensional classification. However, they ignored the covariance structure of the features and the classifiers from those methods were not asymptotically optimal. Some methods have been proposed for covariance matrix estimation or precision matrix estimation in high dimension scenario (see Bickel and Levina 
$[4,5]$, Rothman et al. [37], Cai and Zhang [8], Cai et al. [10]). The covariance matrix estimated by these methods can be directly used in LDA and address the singularity of $\hat{\Sigma}$. However, an accurate estimate of $\Sigma$ does not necessarily lead to better classification. Fan and Fan [15] and Shao et al. [38] showed that even though the true covariance matrix is known, the classification could be no better than random guess because of the noise accumulated from estimating the means. This arouses the second challenge for high dimensional LDA. That is, the noise introduced by the estimation of many non-informative features would lead to poor classification performance. Therefore, the regulation of features is needed.

To address the second challenge stated above, Witten and Tibshirani [42] proposed penalized LDA by applying penalties on the feature vectors. Cai and Liu [7], Mai, Zou and Yuan [30] and Fan, Feng and Tong [16] assumed sparsity and introduced penalization on the discriminant direction $\boldsymbol{\zeta}=\Sigma^{-1}\left(\boldsymbol{\mu}_{2}-\boldsymbol{\mu}_{1}\right)$, which was also adopted by Cai and Zhang [9]. These methods regularized the estimated discriminant direction $\zeta$ directly, avoiding of estimating $\Sigma^{-1}$. The advantages are obvious. The penalization reduced the noise accumulated in estimation on high dimensional features. The obtained classifiers incorporated the covariance structure among features. However, the disadvantage is that the panelized results were not straightforward to interpret. In particular, the panelized directions did not convey any information on which features should be selected. Because we know feature selection is more relevant to the research question. In this paper, we adopt another types of work that assume sparsity on the feature difference $\boldsymbol{\Delta}=\boldsymbol{\mu}_{2}-\boldsymbol{\mu}_{1}$ (the difference of means between the two classes). Shao et al. [38] assumed sparsity and put hard threshold on both the feature difference $\boldsymbol{\Delta}$ and the covariance matrix $\Sigma$. This method provided an asymptotically optimal classifier. The double threshold method in Shao et al. [38] was then extended to quadratic discriminant analysis in $\mathrm{Li}$ and $\mathrm{Shao}$ [26]. Xu et al. [45] proposed a covariance-enhanced method to achieve feature selection for linear discriminant analysis. However, this method did not work directly on selecting informative features. Most recently, Cannings and Samworth [11] used random projection to perform dimension reduction to address this issue. However, this method did not work well when the data is sparse in ultrahigh dimensional settings, say $p$ is in the thousands while sample size $n$ ranges from 50 to 1000 .

In this article, we develop a new classification procedure, named as Penalized Maximum Likelihood Estimation LDA (PMLE-LDA), for high dimensional data with spatially dependent features. We assume that the features follow multivariate normal distribution. We structure the covariance matrix by a spatial covariance function (e.g. Matérn covariance function). By introducing the spatial structure, the covariance matrix can be estimated by maximum likelihood estimation no matter how large the number of features is, compared to the sample size. This estimated covariance matrix can address the first challenge of covariance singularity. To address the second challenge of mean misidentification, we assume that the feature difference between two classes is sparse, which indicates that only a fraction of the $p$ features contribute in differentiating the two classes. Given a training data set, we use Penalized Maximum Likelihood 
Estimation (PMLE) method to perform parameter estimation. The resulting estimates are plugged into LDA model to construct a new classifier. We show in theory that the proposed procedure can not only provide consistent results of parameter estimation and feature selection, but also generate an asymptotically optimal classifier driven by high dimensional data with specific spatially dependent structure. To the best of our knowledge, the proposed method is the first to use spatial correlations to adjust the classification rules. Although we develop PMLE-LDA under the linear framework for two classes classification, it could be potentially extended to other classification methods such as quadratic discriminant analysis (QDA) and multi-classes classification problems.

The rest of the paper is organized as follows. In Section 2, we show that MLELDA is asymptotically optimal if $p / n \rightarrow 0$ under some regularity conditions. We also show that in high dimensional setting $(p / n \rightarrow C>0)$, the MLE-LDA performs poorly (no better than random guess) even if the true covariance is known unless the signals are very strong. This indicates the necessity of penalization for the MLE. In Section 3, we propose to estimate the parameters by penalized MLE (PMLE) by applying a penalty on $\boldsymbol{\Delta}=\boldsymbol{\mu}_{1}-\boldsymbol{\mu}_{2}$, which measures the difference of the two classes in mean. We assume the sparsity of $\boldsymbol{\Delta}$. Then we derive and prove the parameter estimation consistency and feature selection consistency of PMLE. In the end, PMLE-LDA is constructed. We show that it is asymptotically optimal even if $p / n \rightarrow C>0$. Simulation study and real data analysis are conducted in Section 4 and 5 . We conclude and discuss in Section 6 .

\section{Classification using maximum likelihood estimate (MLE-LDA)}

\subsection{Spatial models}

In this section, we introduce necessary terminologies and assumptions in spatial statistics. For a spatial domain of interest $D$ in $\mathbb{R}^{d}$, we consider two classes of spatial processes $\left\{y_{k}(s): s \in D, k=1,2\right\},(k=1,2)$, such that

$$
y_{k}(s)=\mu_{k}(s)+\epsilon(s),
$$

where $\mu_{k}(s)$ is the mean effect function and $\epsilon(s)$ is the corresponding random noise. Assume that the error process $\{\epsilon(s): s \in D\}$ is a Gaussian process with mean zero and a covariance function

$$
\gamma\left(s, s^{\prime} ; \boldsymbol{\theta}\right)=\operatorname{cov}\left(\epsilon(s), \epsilon\left(s^{\prime}\right)\right),
$$

where $s, s^{\prime} \in D$ and $\boldsymbol{\theta}$ is a $q \times 1$ vector of covariance function parameters. We assume that the spatial domain is expanding as the number of samples on the domain is increasing.

Assumption 1. Assume the sample set $D \in \mathbb{R}^{d}(d \geq 1)$ is predetermined and non-random with the restriction $\left\|s_{i}-s_{j}\right\|_{2} \geq \epsilon>0$, for $s_{i}, s_{j} \in D$ for all pairs $i, j=1,2, \ldots, p$ to ensure that the sampling domain increases in extent as $p$ increases. 
Assume for any sample of the spatial processes, there are observations at $p$ discrete sites $s_{1}, \ldots, s_{p} \in D$. Suppose $y_{k i}(s)\left(i=1,2, \ldots, n_{k}\right)$ is from class $\mathcal{C}_{k}$ $(k=1,2)$. Let $Y_{k i j}=y_{k i}\left(s_{j}\right)$ be the observation at $j$ th site for the $i$ th sample of spatial process $y_{k}(s)$, where $k=1,2, i=1,2, \ldots, n_{k}, j=1,2, \ldots, p$, then the $j$ th observation for sample $i$ can be represented by

$$
Y_{k i j}=\mu_{k j}+\epsilon_{k i j}
$$

where $\mu_{k j}=\mu_{k}\left(s_{j}\right)$ is the mean effect at $j$ th location in class $\mathcal{C}_{k}$ and $\epsilon_{k i j}=$ $\epsilon_{k i}\left(s_{j}\right)$ is the corresponding Gaussian random noise for $i$ th sample at $j$ th location. In matrix notation, the above model can be written as

$$
\boldsymbol{Y}_{k i}=\boldsymbol{\mu}_{k}+\boldsymbol{\epsilon}_{k i}
$$

where $\boldsymbol{Y}_{k i}=\left(Y_{k i 1}, \ldots, Y_{k i p}\right)^{T}, \boldsymbol{\mu}_{k}=\left(\mu_{k 1}, \ldots, \mu_{k p}\right)^{T}$ is the mean vector of class $\mathcal{C}_{k}$ and $\epsilon_{k i}=\left(\epsilon_{k i 1}, \ldots \epsilon_{k i p}\right)^{T}$ has multivariate nomal distribution $N(\mathbf{0}, \Sigma)$. As $\epsilon(s)$ has a covariance function (2.2), the covariance matrix $\Sigma$ can be represented by $\Sigma(\boldsymbol{\theta})=\left[\gamma\left(s_{i}, s_{j} ; \boldsymbol{\theta}\right)\right]_{i, j=1}^{p}$, i.e. $\gamma\left(s_{i}, s_{j}\right)$ is the $(i, j)$ th entry. From $(2.4)$, we have

$$
\boldsymbol{Y}_{k i} \sim N\left(\boldsymbol{\mu}_{k}, \Sigma(\boldsymbol{\theta})\right)
$$

Assume $\boldsymbol{\theta}_{0}$ be the true parameter in (2.2). If $\boldsymbol{\theta}=\boldsymbol{\theta}_{0}$, we write $\Sigma\left(\boldsymbol{\theta}_{0}\right)$ as $\Sigma$ for simplicity. Next, we make some assumptions on the covariance function $\gamma\left(s_{i}, s_{j} ; \boldsymbol{\theta}\right)$ :

Assumption 2. (i) Let $\Xi$ be the parameter space for $\boldsymbol{\theta}$. Assume the covariance function $\gamma\left(s, s^{\prime} ; \boldsymbol{\theta}\right)$ is stationary, isotropy, and twice differentiable with respect to $\boldsymbol{\theta}$ for all $\boldsymbol{\theta} \in \Xi$ and $s, s^{\prime} \in D$.

(ii) $\gamma\left(s, s^{\prime} ; \boldsymbol{\theta}\right)$ is positive-definite in the sense that the covariance matrix $\Sigma=$ $\left[\gamma\left(s_{i}, s_{j} ; \boldsymbol{\theta}\right)\right]$ is positive-definite for every finite subset $\left\{s_{1}, s_{2}, \ldots, s_{p}\right\}$ of $D$.

Under the stationary and isotropic assumption, $\Sigma(\boldsymbol{\theta})$ could be written as $\Sigma(\boldsymbol{\theta})=\left[\gamma\left(h_{i j} ; \boldsymbol{\theta}\right)\right]_{i, j=1}^{p}$, where $h_{i j}=\left\|s_{i}-s_{j}\right\|_{2}$ is the Euclidean distance between sites $s_{i}$ and $s_{j}$.

There are many ways to model the covariance function $\gamma(h ; \boldsymbol{\theta})$. A widely used family of covariance function is the Matérn covariance function. It is defined as:

$$
\gamma\left(h ; \sigma^{2}, c, \nu, r\right):=\sigma^{2}(1-c) \frac{2^{1-\nu}}{\Gamma(\nu)}(h / r)^{\nu} K_{\nu}(h / r)
$$

where $K_{\nu}(\cdot)$ is a modified Bessel function of the second kind and $\sigma^{2}>0$ is the variance, $0 \leq c \leq 1$ is a nugget effect, $\nu>0$ is the scale and smoothness parameter (Cressie [14]). First, the Matérn covariance function is isotropic and the correlation decreases when the distance $h$ increases. Second, when $\nu$ increases, the smoothness of the random field increases. Moreover, the Matérn covariance function converges to Gaussian covariance function $\gamma\left(h ; \sigma^{2}, c, r\right)=$ $\sigma^{2}(1-c) \exp \left(-h^{2} / r^{2}\right)$ as $\nu \rightarrow \infty$. Last, if $\nu=\frac{1}{2},(2.6)$ is reduced to the well known exponential covariance function $\gamma\left(h ; \sigma^{2}, c, r\right)=\sigma^{2}(1-c) \exp (-h / r) . r$ is called the range parameter since it measures the distance at which the correlation have decreased below certain threshold. 


\subsection{Classification using MLE-LDA}

Under the setting of spatial statistics, the covariance structures $\Sigma$ and means $\boldsymbol{\mu}_{1}, \boldsymbol{\mu}_{2}$ and $\boldsymbol{\Delta}=\boldsymbol{\mu}_{1}-\boldsymbol{\mu}_{2}$ can be estimated by maximum likelihood estimation (MLE). Plugging the MLE into (1.1) results in a MLE-LDA classifier. In this section, we investigate the properties of MLE-LDA. We prove that MLE-LDA is asymptotically optimal if $p / n \rightarrow 0$ but perform poorly while $p / n \rightarrow C>0$ even if the true covariance is known.

Let $\boldsymbol{Y}=\left(\boldsymbol{Y}_{11}^{T}, \ldots, \boldsymbol{Y}_{1 n_{1}}^{T}, \boldsymbol{Y}_{21}^{T}, \ldots, \boldsymbol{Y}_{2 n_{2}}^{T}\right)^{T}$, where $\boldsymbol{Y}_{k i}(k=1,2$ and $i=$ $\left.1,2, \ldots, n_{k}\right)$ is defined as in Section 2.1. As defined in (2.5), $\boldsymbol{Y}$ is an $n \times p$ dimension vector and follows a multivariate normal distribution. Then we have the log-likelihood function for $\boldsymbol{\mu}_{k}$ and $\boldsymbol{\theta}$

$$
\begin{aligned}
L\left(\boldsymbol{\theta}, \boldsymbol{\mu}_{1}, \boldsymbol{\mu}_{2} ; \boldsymbol{Y}\right)= & -\frac{p\left(n_{1}+n_{2}\right)}{2} \log (2 \pi)-\frac{n_{1}+n_{2}}{2} \log |\Sigma(\boldsymbol{\theta})| \\
& -\frac{1}{2} \sum_{k=1}^{2} \sum_{i=1}^{n_{k}}\left(\boldsymbol{Y}_{k i}-\boldsymbol{\mu}_{k}\right)^{T} \Sigma(\boldsymbol{\theta})^{-1}\left(\boldsymbol{Y}_{k i}-\boldsymbol{\mu}_{k}\right) .
\end{aligned}
$$

We can estimate $\boldsymbol{\mu}_{1}, \boldsymbol{\mu}_{2}$ and $\boldsymbol{\theta}$ by MLE even in high dimensional settings. According to the setting of the spatial model in Section 2.1, the resulting $\Sigma(\hat{\boldsymbol{\theta}})$ is a positive definite matrix. This resolves the first challenge of sample covariance singularity.

We denote the resulting estimates as $\hat{\boldsymbol{\mu}}_{1 M L E}, \hat{\boldsymbol{\mu}}_{2 M L E}, \hat{\boldsymbol{\theta}}_{M L E}$, which can be plugged in (1.1) and get the MLE-LDA classifier $\hat{\delta}_{M L E}$ :

$$
\hat{\delta}_{M L E}(\boldsymbol{\Omega})=\left(\boldsymbol{\Omega}-\frac{\hat{\boldsymbol{\mu}}_{1 M L E}+\hat{\boldsymbol{\mu}}_{2 M L E}}{2}\right)^{T} \Sigma^{-1}\left(\hat{\boldsymbol{\theta}}_{M L E}\right)\left(\hat{\boldsymbol{\mu}}_{1 M L E}-\hat{\boldsymbol{\mu}}_{2 M L E}\right) .
$$

In this section, we investigate the consistency of parameter estimation of (2.7). Also we investigate the classification performance of $\hat{\delta}_{M L E}$. Let $\boldsymbol{\mu}_{1}=$ $\left(\mu_{11}, \mu_{12}, \ldots, \mu_{1 p}\right), \boldsymbol{\mu}_{2}=\left(\mu_{21}, \mu_{22}, \ldots, \mu_{2 p}\right)$ and $\boldsymbol{\theta}_{0}=\left(\theta_{01}, \theta_{02}, \ldots, \theta_{0 q}\right)$ be the true parameters. Let $\Sigma_{k}(\boldsymbol{\theta}), k=1,2, \ldots, q$ be the partial derivative of the matrix $\Sigma(\boldsymbol{\theta})$ with respect to $\theta_{k}$, i.e. $\frac{\partial}{\partial \theta_{k}} \Sigma(\boldsymbol{\theta})=\Sigma_{k}(\boldsymbol{\theta})$. Also let $\Sigma^{k}(\boldsymbol{\theta}), k=1,2, \ldots, q$ denote the partial derivative of the matrix $\Sigma(\boldsymbol{\theta})^{-1}$ with respect to $\theta_{k}$, i.e. $\frac{\partial}{\partial \theta_{k}} \Sigma^{-1}(\boldsymbol{\theta})=\Sigma^{k}(\boldsymbol{\theta})$. Also, denote $\Sigma_{k j}(\boldsymbol{\theta})=\frac{\partial \Sigma(\boldsymbol{\theta})}{\partial \theta_{k} \partial \theta_{j}}$ and $\Sigma^{k j}(\boldsymbol{\theta})=\frac{\partial \Sigma^{-1}(\boldsymbol{\theta})}{\partial \theta_{k} \partial \theta_{j}}$. We are going to simplify the notation if $\boldsymbol{\theta}=\boldsymbol{\theta}_{0}$, i.e. we write $\Sigma\left(\boldsymbol{\theta}_{0}\right)$ as $\Sigma, \Sigma^{-1}\left(\boldsymbol{\theta}_{0}\right)$ as $\Sigma^{-1}, \Sigma_{k}\left(\boldsymbol{\theta}_{0}\right)$ as $\Sigma_{k}$ and $\Sigma^{k}\left(\boldsymbol{\theta}_{0}\right)$ as $\Sigma^{k}$. For a square matrix $A$, denote the set of all the eigenvalues by $\lambda(A)$. Moreover, denote the maximum and minimum eigenvalues by $\lambda_{\max }(A)$ and $\lambda_{\min }(A)$, respectively. 
We need to assume some regularity conditions for Theorem 2.1.

Assumption 3. $\limsup _{p \rightarrow \infty} \lambda_{\max }(\Sigma)<\infty, \liminf _{p \rightarrow \infty} \lambda_{\min }(\Sigma)>0$

Assumption 4. $\left\|\Sigma_{k}\right\|_{F}^{-2}=O_{p}\left(p^{-1}\right)$, where $\left\|\Sigma_{k}\right\|_{F}=\sum_{i, j=1}^{p} \gamma_{k}^{2}\left(h_{i j} ; \boldsymbol{\theta}\right)$, where $\gamma_{k}\left(h_{i j} ; \boldsymbol{\theta}\right)=\frac{\partial \gamma\left(h_{i j} ; \boldsymbol{\theta}\right)}{\partial \theta_{k}}, k=1,2, \ldots, q$ and $\boldsymbol{\theta}$ is a $k$ dimensional parameter.

Assumption 5. Assume $\lim _{p \rightarrow \infty} a_{i j}$ exist, where $a_{i j}=\frac{t_{i j}}{t_{i i}^{1 / 2} t_{j j}^{1 / 2}}$ and $t_{i j}=$ $\operatorname{tr}\left(\Sigma^{-1} \Sigma_{i} \Sigma^{-1} \Sigma_{j}\right)$

Assumption 6. There exists an open subset $\omega$ that contains the true parameter point $\boldsymbol{\theta}_{0}$ such that for all $\boldsymbol{\theta}^{*} \in \omega$, we have:

(i)

$-\infty<\lim _{p \rightarrow \infty} \lambda_{\min }\left(\Sigma_{k}\left(\boldsymbol{\theta}^{*}\right)\right)<\lim _{p \rightarrow \infty} \lambda_{\max }\left(\Sigma_{k}\left(\boldsymbol{\theta}^{*}\right)\right)<\infty$.

$-\infty<\lim _{p \rightarrow \infty} \lambda_{\min }\left(\Sigma_{k j}\left(\boldsymbol{\theta}^{*}\right)\right)<\lim _{p \rightarrow \infty} \lambda_{\max }\left(\Sigma_{k j}\left(\boldsymbol{\theta}^{*}\right)\right)<\infty$.

$\left\|\frac{\partial t_{i j}\left(\boldsymbol{\theta}^{*}\right)}{\partial \boldsymbol{\theta}}\right\|_{2}=O_{p}(p)$, where $t_{i j}\left(\boldsymbol{\theta}^{*}\right)=\operatorname{tr}\left(\Sigma^{-1}\left(\boldsymbol{\theta}^{*}\right) \Sigma_{i}\left(\boldsymbol{\theta}^{*}\right) \Sigma^{-1}\left(\boldsymbol{\theta}^{*}\right) \Sigma_{j}\left(\boldsymbol{\theta}^{*}\right)\right)$

Since $\Sigma^{k}=-\Sigma \Sigma_{k} \Sigma$ and $\Sigma^{k j}=\Sigma^{-1}\left(\Sigma_{k} \Sigma^{-1} \Sigma_{j}+\Sigma_{j} \Sigma^{-1} \Sigma_{k}-\Sigma_{k j}\right) \Sigma^{-1}$, by Assumption 3 and Assumption 6, we have

$$
-\infty<\lim _{p \rightarrow \infty} \lambda_{\min }\left(\Sigma^{k}\left(\boldsymbol{\theta}^{*}\right)\right)<\lim _{p \rightarrow \infty} \lambda_{\max }\left(\Sigma^{k}\left(\boldsymbol{\theta}^{*}\right)\right)<\infty
$$

and

$$
-\infty<\lim _{p \rightarrow \infty} \lambda_{\min }\left(\Sigma^{k j}\left(\boldsymbol{\theta}^{*}\right)\right)<\lim _{p \rightarrow \infty} \lambda_{\max }\left(\Sigma^{k j}\left(\boldsymbol{\theta}^{*}\right)\right)<\infty .
$$

Notice that for any $p \times p$ matrix $A$ we have $\|A\|_{F} \leq \sqrt{p}\|A\|_{2}=\sqrt{p} \lambda_{\max }(A)$, then from Assumption 6 we have:

(1) $\left\|\Sigma^{k}\left(\boldsymbol{\theta}^{*}\right)\right\|_{F}=O_{p}(\sqrt{p})$;

(2) $\left\|\Sigma^{k j}\left(\boldsymbol{\theta}^{*}\right)\right\|_{F}=O_{p}(\sqrt{p})$.

First we have the following theorem about MLE consistency of (2.7).

Theorem 2.1. Assume Assumptions 2-6 hold. Let $\left(\boldsymbol{\mu}_{1}, \boldsymbol{\mu}_{2}, \boldsymbol{\theta}_{0}\right)$ be the true parameter. The maximum likelihood estimate (MLE) of (2.7) is: $\hat{\boldsymbol{\mu}}_{1 M L E}=\overline{\boldsymbol{Y}}_{1}$, $\hat{\boldsymbol{\mu}}_{2 M L E}=\overline{\boldsymbol{Y}}_{2 .}, \hat{\boldsymbol{\theta}}_{M L E}$, where $\overline{\boldsymbol{Y}}_{k} .=\sum_{i=1}^{n_{k}} \boldsymbol{Y}_{k i} / n_{k}$. Also,

(i) If $p / n \rightarrow 0,\left\|\hat{\boldsymbol{\theta}}_{M L E}-\boldsymbol{\theta}_{0}\right\|_{2}=O_{p}\left(\frac{1}{\sqrt{n p}}\right)$;

(ii) If $p / n \rightarrow C$ with $0<C \leq \infty$ and $\sqrt{p} / n \rightarrow 0$, $\left\|\hat{\boldsymbol{\theta}}_{M L E}-\boldsymbol{\theta}_{0}\right\|_{2}=O_{p}\left(\frac{1}{n}\right)$.

Proof. See Appendix A.1.

In Theorem 2.1, Assumption 2 is necessary to ensure the good property for covariance matrix $\Sigma(\boldsymbol{\theta})$. Assumption 3 and Assumption 5 are assumed in Mardia and Marshall [31] for existence of MLE for spatial regression for fixed $p$ when $n \rightarrow \infty$. Assumption 4 and Assumption 6 are necessary conditions for the 
parameter consistency when $p \rightarrow \infty$. It is obvious that Matérn covariance function satisfies Assumption 2. In the section of Remarks on the assumptions 7,we verified the Matérn covariance function satisfy the first part of Assumption 3 and Assumptions 4-6. Matérn covariance function also satisfy the second part of Assumption 3 if Assumption 1 holds. Theorem 2.1 shows that under the spatial statistical model, all the parameters $\left(\boldsymbol{\mu}_{1}, \boldsymbol{\mu}_{2}, \boldsymbol{\theta}\right)$ can be estimated consistently by the MLE for either $p / n \rightarrow 0$ or $p / n$ goes to a positive constant or $\infty$. Therefore we obtain a positive definite covariance matrix estimate of $\Sigma(\boldsymbol{\theta})$. We can then plug-in the MLE's, $\hat{\boldsymbol{\mu}}_{1}=\hat{\boldsymbol{\mu}}_{1 M L E}, \hat{\boldsymbol{\mu}}_{2}=\hat{\boldsymbol{\mu}}_{2 M L E}$ and $\hat{\Sigma}=\Sigma\left(\hat{\boldsymbol{\theta}}_{M L E}\right)$ into (1.7) to build up the classification function (2.8).

Then a new observation $\boldsymbol{\omega}$ of $\boldsymbol{\Omega}$ would be classified into class $\mathcal{C}_{1}$ if $\hat{\delta}_{M L E}(\boldsymbol{\omega})>$ 0 and $\mathcal{C}_{2}$ otherwise. Using the same notations in Section 1 , the conditional misclassification rate is defined by (1.2) and (1.4). For simplicity, We are going to use $\hat{\boldsymbol{\mu}}_{1}$ to denote $\hat{\boldsymbol{\mu}}_{1 M L E}, \hat{\boldsymbol{\mu}}_{2}$ to denote $\hat{\boldsymbol{\mu}}_{2 M L E}, \hat{\boldsymbol{\theta}}$ to denote $\hat{\boldsymbol{\theta}}_{M L E}$ in this section.

We will see in Theorem 2.2 that the approximate optimal error rate can be achieved while $p / n \rightarrow 0$. However, Theorem 2.3 shows if $p / n \rightarrow C$ with $0<C \leq \infty$, the error rate would be no better than random guessing even if we know the true covariance, due to the error accumulated in the estimation of $\boldsymbol{\mu}_{1}$ and $\boldsymbol{\mu}_{2}$.

Theorem 2.2. Let $C_{p}=\boldsymbol{\Delta}^{T} \Sigma(\boldsymbol{\theta}) \boldsymbol{\Delta}$. Assume $\frac{p}{n} \rightarrow 0, C_{p} \rightarrow C_{0}$ with $0 \leq C_{0} \leq$ $\infty$, and $n C_{p} \rightarrow \infty$ as $n, p \rightarrow \infty$.

(1) The overall misclassification rate $W\left(\hat{\delta}_{M L E}\right)$ is asymptotically sub-optimal. In other words, $W\left(\hat{\delta}_{M L E}\right) \rightarrow 1-\Phi\left(\frac{\sqrt{C_{0}}}{2}\right)$.

(2) Moreover, if $C_{p} \rightarrow C_{0}$ with $0 \leq C_{0}<\infty$ or if $C_{p} \rightarrow \infty$ and $C_{p} \frac{p}{n} \rightarrow 0$, then $W\left(\hat{\delta}_{M L E}\right)$ is asymptotically optimal, i.e. $\frac{W\left(\hat{\delta}_{M L E}\right)}{W_{O P T}} \stackrel{P}{\rightarrow} 1$.

Proof. See Appendix A.1.

The following theorem shows that while $\frac{p}{n}$ goes to a positive constant or $\infty$, even though the true covariance is known, the error accumulated in the estimation of $\boldsymbol{\mu}_{1}$ and $\boldsymbol{\mu}_{2}$ would cause biased misclassification rate unless the signal levels $\left(C_{p}\right)$ are extremely high. This discovery suggests that even though there's no problem in parameter estimation in our model even in high dimensional case, it is still necessary to select important features for classification.

Theorem 2.3. Assume the true covariance $\Sigma$ is known, we denote the classifier function as

$$
\delta_{\hat{\boldsymbol{\mu}}}(\boldsymbol{\omega})=(\boldsymbol{\omega}-\hat{\boldsymbol{\mu}})^{T} \Sigma^{-1}\left(\hat{\boldsymbol{\mu}}_{1}-\hat{\boldsymbol{\mu}}_{2}\right)
$$

where $\hat{\boldsymbol{\mu}}_{1}, \hat{\boldsymbol{\mu}}_{2}$ are MLE in (2.7) and $\hat{\boldsymbol{\mu}}=\frac{\hat{\boldsymbol{\mu}}_{1}+\hat{\boldsymbol{\mu}}_{2}}{2}$. Assume $p / n \rightarrow C$ with $0<$ $C \leq \infty, C_{p} \rightarrow C_{0}$ with $0 \leq C_{0} \leq \infty$. Assume $n_{1} \neq n_{2}$ and $n_{k}>\frac{n}{4}(k=1,2)$, then

(1) For $\frac{C_{p}}{p / n} \rightarrow \infty$, then $W\left(\hat{\delta}_{\hat{\boldsymbol{\mu}}}\right) \stackrel{P}{\rightarrow} 0$ and $W_{O P T} \stackrel{P}{\rightarrow} 0$ but $\frac{W\left(\hat{\delta}_{\hat{\mu}}\right)}{W_{O P T}} \stackrel{P}{\rightarrow} \infty$. 
(2) For $\frac{C_{p}}{p / n} \rightarrow c$ with $0<c<\infty$,

(i) if $\frac{p}{n} \rightarrow C<\infty$, then $\lim _{P} W\left(\hat{\delta}_{\hat{\boldsymbol{\mu}}}\right)>1-\Phi\left(\frac{\sqrt{C_{0}}}{2}\right)$;

(ii) if $\frac{p}{n} \rightarrow \infty$, then $W\left(\hat{\delta}_{\hat{\boldsymbol{\mu}}}\right) \stackrel{P}{\rightarrow} 0$ and $W_{O P T} \stackrel{P}{\rightarrow} 0$, but $\frac{W\left(\hat{\delta}_{\hat{\mu}}\right)}{W_{O P T}} \stackrel{P}{\rightarrow} \infty$.

(3) For $\frac{C_{p}}{p / n} \rightarrow 0$, then $W\left(\hat{\delta}_{\hat{\mu}}\right) \stackrel{P}{\rightarrow} \frac{1}{2}$.

Proof. See Appendix A.1.

Corollary 1. Providing all the same conditions hold in Theorem 2.3, and if $n_{1}=n_{2}$, then

(1) If $\frac{C_{p}}{\sqrt{p / n}} \rightarrow \infty$, then $W\left(\hat{\delta}_{\hat{\boldsymbol{\mu}}}\right) \stackrel{P}{\rightarrow} 0$ and $W_{O P T} \stackrel{P}{\rightarrow} 0$, but $\frac{W\left(\hat{\delta}_{\hat{\mu}}\right)}{W_{O P T}} \stackrel{P}{\rightarrow} \infty$;

(2) If $\frac{C_{p}}{\sqrt{p / n}} \rightarrow c$ with $0<c<\infty$,

(i) If $\frac{p}{n} \rightarrow C$, then $W\left(\hat{\delta}_{\hat{\boldsymbol{\mu}}}\right) \stackrel{P}{\rightarrow} 1-\Phi\left(\frac{c}{2 \sqrt{4+c / \sqrt{C}}}\right)$ and $W_{O P T} \stackrel{P}{\rightarrow} 1-$ $\Phi\left(\frac{\sqrt{c \sqrt{C}}}{2}\right)$

(ii) If $\frac{p}{n} \rightarrow \infty$, then $W\left(\hat{\delta}_{\hat{\mu}}\right) \stackrel{P}{\rightarrow} 1-\Phi\left(\frac{c}{4}\right)$, and $W_{O P T} \stackrel{P}{\rightarrow} 0$;

(3) If $\frac{C_{p}}{\sqrt{p / n}} \stackrel{P}{\rightarrow} 0$, we have $W\left(\hat{\delta}_{\hat{\boldsymbol{\mu}}}\right) \stackrel{P}{\rightarrow} \frac{1}{2}$.

Proof. See Appendix A.1.

Theorem 2.3 and the Corollary 1 show that while $p / n \rightarrow C$ with $0<C \leq \infty$, $\hat{\delta}_{\hat{\mu}}$ is never asymptotically optimal. It is asymptotically sub-optimal only if $C_{p} \rightarrow \infty$. We can apply LDA to spatially dependent data when estimating the parameters by MLE, however, in high dimensional case $(p / n \rightarrow C$ with $0<C \leq$ $\infty)$, the classification performance may be poor due to noise accumulated in the estimation of $\boldsymbol{\mu}_{1}$ and $\boldsymbol{\mu}_{2}$ (see Fan and Fan [15] and Shao et al. [38]). Therefore, feature selection is still critical for classification with high dimension. Fan and Fan [15] seeks to extract salient features by two-sample t-test and proved that ttest can pick up all important features by choosing an appropriate critical value once the features are assumed to be independent. Shao et al. [38] proposes to select features by threshold. In this paper, we propose to use penalized maximum likelihood estimates (PMLE) for parameter estimation and feature selection, when the features are spatially correlated.

\section{Classification using penalized maximum likelihood estimate (PMLE-LDA)}

\subsection{The penalized maximum likelihood estimation (PMLE)}

In this section, we consider feature selection for the high dimensional classification problem (i.e. $p / n \rightarrow C$ with $0<C \leq \infty$ as $p \rightarrow \infty$ and $n \rightarrow \infty$ ). We recall the notation used in Section 1 . We use $\left(\Delta_{1}, \ldots, \Delta_{2}\right)=\left(\mu_{21}-\mu_{11}, \ldots, \mu_{2 p}-\mu_{1 p}\right)$ 
to denote the differences of the mean between class $\mathcal{C}_{1}$ and $\mathcal{C}_{2}$. The vector form is denoted as $\boldsymbol{\Delta}=\boldsymbol{\mu}_{2}-\boldsymbol{\mu}_{1}$, which is a $p$ dimensional vector. Define the signal set $S=\left\{j: \Delta_{j} \neq 0\right\}$. Let $s$ be the number of non zero elements in $\boldsymbol{\Delta}$. The important features are contained in the set $S$. Instead of assuming the sparsity of discriminant direction (Cai and Liu [7], Fan, Feng and Tong [16], Mai, Zou and Yuan $[30]$ ), we assume the sparsity of feature difference $\boldsymbol{\Delta}$ (i.e. $s \ll n$ and $s / n \rightarrow 0$ ). Next, we derive the penalized likelihood function based on the assumption that the observations $\boldsymbol{Y}_{k i}$ are normally distributed $\boldsymbol{Y}_{k i} \sim N\left(\boldsymbol{\mu}_{k}, \Sigma\left(\boldsymbol{\theta}_{0}\right)\right)$ for $k=1,2$ and $i=1,2, \ldots, n_{k}$.

First, we define two matrix forms that help simplify notations in subsequent derivations. Let $I_{p}$ be a $p \times p$ identity matrix. We denote the diagonal block matrix for square matrix $A$ as $\operatorname{diag}_{n}(A)$. We denote the block matrix for identity matrix $I_{p}$ as $\tilde{J}_{n, p}$. Their definitions are given as follows.

$$
\operatorname{diag}_{n}(A)=\underbrace{\left(\begin{array}{cccc}
A & 0 & \cdots & 0 \\
0 & A & \cdots & 0 \\
\vdots & \vdots & \ddots & \vdots \\
0 & 0 & 0 & A
\end{array}\right)}_{n \times n \text { blocks }}, \tilde{J}_{n, p}=\underbrace{\left(\begin{array}{cccc}
I_{p} & I_{p} & \cdots & I_{p} \\
I_{p} & I_{p} & \cdots & I_{p} \\
\vdots & \vdots & \ddots & \vdots \\
I_{p} & I_{p} & I_{p} & I_{p}
\end{array}\right)}_{n \times n \text { blocks }} .
$$

Both $\operatorname{diag}_{n}(A)$ and $\tilde{J}_{n, p}$ consist of $n \times n$ blocks. Thus we define $\tilde{I}_{n, p}=\operatorname{diag}_{n}\left(I_{p}\right)$.

Recall that $\boldsymbol{Y}=\left(\boldsymbol{Y}_{11}^{T}, \cdots, \boldsymbol{Y}_{1 n_{1}}^{T}, \boldsymbol{Y}_{21}^{T}, \cdots, \boldsymbol{Y}_{2 n_{2}}^{T}\right)^{T}$ is an $n \times p$ dimensional vector. In order to estimate $\boldsymbol{\Delta}=\boldsymbol{\mu}_{1}-\boldsymbol{\mu}_{2}$, we transform $\boldsymbol{Y}$ by letting $\mathbf{Z}=\mathbf{V} \boldsymbol{Y}$, where $\mathbf{V}$ is a $(n-1) p \times n p$ matrix made up of the first $(n-1) p$ rows of $\tilde{I}_{n, p}-\frac{1}{n} \tilde{J}_{n, p}$. Then $\mathbf{Z}=\left(\begin{array}{l}\mathbf{Z}_{1}^{T} \mathbf{Z}_{2}^{T} \cdots \mathbf{Z}_{n-1}^{T}\end{array}\right)^{T}$, where $\mathbf{Z}_{i}=\boldsymbol{Y}_{1 i}-\overline{\boldsymbol{Y}}$ for $i=1,2, \ldots, n_{1}, \mathbf{Z}_{i}=$ $\boldsymbol{Y}_{2\left(i-n_{1}\right)}-\overline{\boldsymbol{Y}}$ for $i=n_{1}+1, n_{1}+2, \ldots, n-1$ and $\overline{\boldsymbol{Y}}=\frac{1}{n} \sum_{k=1}^{2} \sum_{i=1}^{n_{k}} \boldsymbol{Y}_{k i}$. Note that transformed data $\mathbf{Z}$ becomes $(n-1) p$ dimensional instead of $n p$ dimensional, because it is known that the freedom of a centered transformation is $n-1$ (if performed on $n$ observations). Then the distribution of $\mathbf{Z}$ is given as

$$
\mathbf{Z}_{i} \sim\left\{\begin{array}{l}
N\left(-\tau_{2} \boldsymbol{\Delta}, \frac{n-1}{n} \Sigma\left(\boldsymbol{\theta}_{0}\right)\right), i=1,2, \ldots, n_{1}, \\
N\left(\tau_{1} \boldsymbol{\Delta}, \frac{n-1}{n} \Sigma\left(\boldsymbol{\theta}_{0}\right)\right), i=n_{1}+1, \ldots, n-1 .
\end{array}\right.
$$

where $\tau_{1}=\frac{n_{1}}{n}$ and $\tau_{2}=\frac{n_{2}}{n}$. The covariance is $\operatorname{cov}\left(\mathbf{Z}_{i}, \mathbf{Z}_{j}\right)=-\frac{1}{n} \Sigma$ for $i \neq j$. We define $\boldsymbol{X}^{(1)}$ and $\boldsymbol{X}^{(2)}$ as

$$
\boldsymbol{X}^{(1)}=\left(\begin{array}{cccc}
-\tau_{2} & 0 & \cdots & 0 \\
0 & -\tau_{2} & \cdots & 0 \\
\vdots & \vdots & \ddots & \vdots \\
0 & 0 & 0 & -\tau_{2}
\end{array}\right)_{p \times p}, \quad \boldsymbol{X}^{(2)}=\left(\begin{array}{cccc}
\tau_{1} & 0 & \cdots & 0 \\
0 & \tau_{1} & \cdots & 0 \\
\vdots & \vdots & \ddots & \vdots \\
0 & 0 & 0 & \tau_{1}
\end{array}\right)_{p \times p}
$$

We further define that $\boldsymbol{X}_{i}=\boldsymbol{X}^{(1)}$ for $i=1,2, \ldots, n_{1}$ and $\boldsymbol{X}_{i}=\boldsymbol{X}^{(2)}$ for $i=n_{1}+1, \ldots, n-1$. Then we have $\boldsymbol{X}=\left(\boldsymbol{X}_{1}^{T}, \boldsymbol{X}_{2}^{T}, \cdots, \boldsymbol{X}_{n-1}^{T}\right)^{T}$.

Because PMLE is traditionally used in a linear regression setup, we rewrite $\boldsymbol{\beta}=\boldsymbol{\Delta}$, which keeps the notations that researchers have been familiar with. Then 
the $(n-1) p \times 1$ vector $\mathbf{Z}$ is a multivariate normal distribution $N(\boldsymbol{X} \boldsymbol{\beta}, \dot{\Sigma})$, where $\dot{\Sigma}=\left(\tilde{I}_{n-1, p}-\frac{1}{n} \tilde{J}_{n-1, p}\right) \operatorname{diag}_{n-1}(\Sigma)$. Denote all the unknown parameters by $\boldsymbol{\eta}=$ $(\boldsymbol{\beta}, \boldsymbol{\theta}) \in \mathbb{R}^{p+q}$. Based on the fact that $\left.|\dot{\Sigma}|=\left|\tilde{I}_{n-1, p}-\frac{1}{n} \tilde{J}_{n-1, p}\right| \mid \operatorname{diag}_{n-1} \Sigma(\boldsymbol{\theta})\right) \mid=$ $\left(\frac{1}{n}\right)^{p}|\Sigma(\boldsymbol{\theta})|^{n-1}$ and $\left(\tilde{I}_{n-1, p}-\frac{1}{n} \tilde{J}_{n-1, p}\right)^{-1}=\tilde{I}_{n-1, p}+\tilde{J}_{n-1, p}$, we can write the penalized $\log$-likelihood function of $\boldsymbol{\beta}$ and $\boldsymbol{\theta}$ as

$$
\begin{aligned}
& Q(\boldsymbol{\theta}, \boldsymbol{\beta} ; \mathbf{Z}) \\
= & -\frac{n p}{2} \log (2 \pi)-\frac{1}{2} \log |\dot{\Sigma}|-\frac{1}{2}(\mathbf{Z}-\boldsymbol{X} \boldsymbol{\beta})^{T} \dot{\Sigma}^{-1}(\mathbf{Z}-\boldsymbol{X} \boldsymbol{\beta})-n \sum_{j=1}^{p} P_{\lambda}\left(\left|\beta_{j}\right|\right) \\
= & C_{n, p}-\frac{n-1}{2} \log |\Sigma|-\frac{1}{2}(\mathbf{Z}-\boldsymbol{X} \boldsymbol{\beta})^{T} \operatorname{diag}_{n-1}\left(\Sigma^{-1}\right)\left(\tilde{I}_{n-1, p}+\tilde{J}_{n-1, p}\right)(\mathbf{Z}-\boldsymbol{X} \boldsymbol{\beta}) \\
& -n \sum_{j=1}^{p} P_{\lambda}\left(\left|\beta_{j}\right|\right),
\end{aligned}
$$

where $C_{n, p}=-\frac{(n-1) p}{2} \log \pi+\frac{p}{2} \log n . P_{\lambda}(x)$ is a generic sparsity-inducing penalty, which could be the lasso penalization or folded concave penalization (such as the SCAD and the MCP). We will elaborate the choice of penalization later in this paper.

In the joint likelihood (3.1), we can see that $\boldsymbol{\theta}$ and $\boldsymbol{\beta}$ play different roles because one is included in the mean and the other is included in the covariance. So it is difficult to obtain the estimation of them simultaneously. The exact solution of (3.1) should be achieved through numerous iterations before convergence. However, to save computational time, we adopt the one-step estimation procedure to estimate $\boldsymbol{\theta}$ and $\boldsymbol{\beta}$ though iterative updates (Chu et al. [12]). The procedure is shown as follows.

\section{One-step PMLE (PMLE $\left.{ }_{o s e}\right)$ computing procedure:}

1. Initialize $\boldsymbol{\beta}$ by minimizing $R(\boldsymbol{\beta})=(\mathbf{Z}-\boldsymbol{X} \beta)^{T}(\mathbf{Z}-\boldsymbol{X} \beta)+$ $n \sum_{j=1}^{p} P_{\lambda}\left(\left|\beta_{j}\right|\right)$ with respect to $\boldsymbol{\beta}$. Denote the initialization by $\hat{\boldsymbol{\beta}}^{(0)}$;

2. With $\boldsymbol{\beta}=\hat{\boldsymbol{\beta}}^{(0)}$, estimate $\boldsymbol{\theta}$ by maximizing $Q\left(\boldsymbol{\theta}, \hat{\boldsymbol{\beta}}^{(0)} ; \mathbf{Z}\right)$ in (3.1) with respect to $\boldsymbol{\theta}$. Denote the estimate by $\hat{\boldsymbol{\theta}}^{(0)}$;

3. With $\boldsymbol{\theta}=\hat{\boldsymbol{\theta}}^{(0)}$, update $\boldsymbol{\beta}$ by maximizing $Q\left(\hat{\boldsymbol{\theta}}^{(0)}, \boldsymbol{\beta} ; \mathbf{Z}\right)$ in $(3.1)$ with respect to $\boldsymbol{\beta}$. Denote the estimate by $\hat{\boldsymbol{\beta}}^{(1)}$;

4. With $\boldsymbol{\beta}=\hat{\boldsymbol{\beta}}^{(1)}$, estimate $\boldsymbol{\theta}$ by maximizing $Q\left(\boldsymbol{\theta}, \hat{\boldsymbol{\beta}}^{(1)} ; \mathbf{Z}\right)$ in (3.1) with respect to $\boldsymbol{\theta}$. Denote the estimate by $\hat{\boldsymbol{\theta}}^{(1)}$.

Then $\hat{\boldsymbol{\theta}}_{\text {ose }}=\hat{\boldsymbol{\theta}}^{(1)}$ and $\hat{\boldsymbol{\beta}}_{\text {ose }}=\hat{\boldsymbol{\beta}}^{(1)}$ are the obtained estimates. We call $\hat{\boldsymbol{\theta}}_{\text {ose }}$ and $\hat{\boldsymbol{\beta}}_{\text {ose }}$ as the one-step PMLE. Mean parameters $\boldsymbol{\mu}_{1}$ and $\boldsymbol{\mu}_{2}$ can be estimated by $\hat{\boldsymbol{\mu}}_{1, \text { ose }}=\overline{\boldsymbol{Y}}-\tau_{2} \hat{\boldsymbol{\beta}}_{\text {ose }}$ and $\hat{\boldsymbol{\mu}}_{2, \text { ose }}=\overline{\boldsymbol{Y}}+\tau_{1} \hat{\boldsymbol{\beta}}_{\text {ose }}$. Besides, we estimate the covariance as $\hat{\Sigma}=\Sigma\left(\hat{\boldsymbol{\theta}}_{\text {ose }}\right)$. The $(i, j)$ th element of $\hat{\Sigma}$ is $\hat{\sigma}_{i, j}=\gamma\left(h_{i j} ; \hat{\boldsymbol{\theta}}_{\text {ose }}\right)$, where $h_{i j}=\left\|s_{j}-s_{i}\right\|_{2}$ is the Euclidean distance between site $s_{i}$ and $s_{j}$. 


\subsubsection{Consistency of one-step PMLE}

Penalty function largely determines the sampling properties of the penalized likelihood estimates. Some additional assumptions about the penality function and tuning parameter $\lambda$ are needed:

Assumption 7. Assume $a_{n}=O_{p}\left(\frac{1}{\sqrt{n}}\right)$, where $a_{n}=\max _{1 \leq j \leq p}\left\{p_{\lambda_{n}}^{\prime}\left(\left|\beta_{0 j}\right|\right)\right.$, $\left.\beta_{0 j} \neq 0\right\}$.

Assumption 8. $b_{n} \rightarrow 0$ as $n \rightarrow \infty$, where $b_{n}=\max _{1 \leq j \leq m}\left\{p_{\lambda_{n}}^{\prime \prime}\left(\left|\beta_{0 j}\right|\right)\right.$, $\left.\beta_{0 j} \neq 0\right\}$.

Assumption 9. $\lambda_{n} \rightarrow 0$ and $\lambda_{n} / \sqrt{\frac{s}{n}} \rightarrow \infty$.

Assumption 10. $\liminf _{\substack{n \rightarrow \infty \\ p \rightarrow \infty}} \liminf _{\theta \rightarrow 0+} P_{\lambda_{n}}^{\prime}(|\theta|) / \lambda_{n}>0$.

Assumption 7 ensures the unbiasedness property for large parameters and the existence of the consistent penalized likelihood estimator. Assumption 8 ensures that the penalty function does not influence the penalized likelihood estimators more than the likelihood function itself. Assumption 10 ensures the penalized likelihood estimators possess the sparsity property. Assumption 9 leads to the variable selection consistency.

Smoothly Clipped Absolute Deviation (SCAD) penalty satisfies all these assumptions. We adopt SCAD penalization in this paper. Fan and Li [17] proposed the SCAD penalty function and claimed that it has three good properties: unbiasedness, sparsity and continuity. Unbiasedness means that there is no overpenalization of large features to avoid unnecessary modeling biases. Sparsity means that the insignificant parameters are set to 0 by a thresholding rule to reduce model complexity. Continuity means that the penalized likelihood produces continuous estimators. The SCAD penalty function is defined as

$$
p_{\lambda}(\beta)= \begin{cases}\lambda|\beta| & \text { if }|\beta| \leq \lambda \\ -\frac{\beta^{2}-2 a \lambda \beta+\lambda^{2}}{2(a-1)} & \text { if } \lambda<|\beta| \leq a \lambda \\ \frac{(a+1) \lambda^{2}}{2} & \text { if }|\beta|>a \lambda\end{cases}
$$

for some $a>0$. More details can be found in Fan and Li [17]. We first illustrate the property of PMLE of (3.1) by the following theorem.

We illustrate the property of one step PMLE as follows. Recall that the true parameter $\boldsymbol{\beta}_{0}$ is a parameter vector of size $p$, and $\boldsymbol{\theta}_{0}=\left(\theta_{01}, \theta_{02}, \ldots, \theta_{0 q}\right)$ is a $q$-dimensional parameter in covariance function. We define the sparsity of $\boldsymbol{\beta}_{0}$ as follows. Without loss of generality, we can write $\boldsymbol{\beta}_{0}=\left(\beta_{1,0}^{T}, \beta_{2,0}^{T}\right)^{T}$, where $\beta_{1,0} \in \mathbb{R}^{s}$ stands for non-zero components, and $\beta_{0,2}=\mathbf{0}_{(p-s) \times 1}$ stands for zero components. The number of nonzero components suffices that $\frac{s}{n} \rightarrow 0$ as $n, p, s \rightarrow$ $\infty$. So we can write $\boldsymbol{X}_{i}=\left(\boldsymbol{X}_{i}^{1}, \boldsymbol{X}_{i}^{2}\right), i=1,2, \ldots, n$, where $\boldsymbol{X}_{i}^{1}$ is the $p \times s$ submatrix of $\boldsymbol{X}_{i}$ made up of nonzero columns in $\operatorname{supp}\left(\boldsymbol{\beta}_{0}\right)$ and $\boldsymbol{X}_{i}^{2}$ is the $p \times(p-$ $s$ ) complement matrix. As demonstrated in Zou and Li [48], the one-step method is as efficient as the fully iterative method both empirically and theoretically, provided that the initial estimators are reasonably good. We will see in the proof 
of Theorem 3.1, in the one step estimation Algorithm stated in Section 3.1, the initial estimators for $\boldsymbol{\beta}\left(\hat{\boldsymbol{\beta}}^{(0)}\right)$ is obtained by minimizing penalized regression function $R(\boldsymbol{\beta})=(\mathbf{Z}-\boldsymbol{X} \beta)^{T}(\mathbf{Z}-\boldsymbol{X} \beta)+n \sum_{j=1}^{p} P_{\lambda}\left(\left|\beta_{j}\right|\right)$, which is consistent and has oracle property. The initial estimator for $\boldsymbol{\theta}\left(\hat{\boldsymbol{\theta}}^{(0)}\right)$ is also a consistent estimate from MLE. As a result, the one-step PMLE (PMLE $\left.{ }_{\text {ose }}\right)$ has good property as demonstrated in Theorem 3.1.

Theorem 3.1. Suppose conditions Assumptions 2-10 hold. Let $\boldsymbol{\beta}_{0}=$ $\left(\boldsymbol{\beta}_{1,0}^{T}, \boldsymbol{\beta}_{2,0}^{T}\right)^{T}$, where $\boldsymbol{\beta}_{1,0} \in \mathbb{R}^{s}$ is non-zero component, $\boldsymbol{\beta}_{2,0}=\boldsymbol{O}_{(p-s) \times 1}$ is the zero component of $\boldsymbol{\beta}_{0}$ with $\frac{s}{n} \rightarrow 0, \frac{p}{n} \rightarrow C$ with $0<C \leq \infty$ as $n, p, s \rightarrow \infty$. The one-step PMLE of (3.1) from the one step procedure in Section 3.1 (PMLE $E_{\text {ose }}$ ) is $\hat{\boldsymbol{\eta}}_{\text {ose }}=\left(\hat{\boldsymbol{\beta}}_{\text {ose }}, \hat{\boldsymbol{\theta}}_{\text {ose }}\right)$ with $\hat{\boldsymbol{\beta}}_{\text {ose }}=\left(\hat{\boldsymbol{\beta}}_{1, \text { ose }}^{T}, \hat{\boldsymbol{\beta}}_{2, \text { ose }}^{T}\right)^{T}$ and $\hat{\boldsymbol{\beta}}_{1, \text { ose }}$ is a sub-vector of $\hat{\boldsymbol{\beta}}_{\text {ose }}$ formed by nonzero components in supp $\left(\boldsymbol{\beta}_{0}\right)$. Then $\hat{\boldsymbol{\eta}}_{\text {ose }}$ satisfy:

(a) (consistency) $\left\|\hat{\boldsymbol{\theta}}_{\text {ose }}-\boldsymbol{\theta}_{0}\right\|_{2}=O_{p}\left(\frac{1}{\sqrt{n p}}\right)$ and $\left\|\hat{\boldsymbol{\beta}}_{\text {ose }}-\boldsymbol{\beta}_{0}\right\|_{2}=O_{p}\left(\sqrt{\frac{s}{n}}\right)$.

(b) (sparsity) $\hat{\boldsymbol{\beta}}_{2, \text { ose }}=0$ with probability tending to 1 as $n \rightarrow \infty$.

Proof. See Appendix A.2.

\subsubsection{Covariance tapering and one-step PMLE}

When the number of features is large ( $p$ is large) for each realization of the spatial process, calculating the likelihood can be computationally infeasible (requiring $\mathcal{O}\left(p^{3}\right)$ calculation). Covariance tapering can be used to approximate the likelihood. When the covariance matrix is replaced with a tapered one, the resulting matrices can then be manipulated using efficient sparse matrix algorithms which would reduce computational burden effectively.

In Section 1, the covariance matrix is defined as $\Sigma(\boldsymbol{\theta})=\left[\gamma\left(s_{i}, s_{j}\right)\right]_{i, j=1}^{p}$. Under Assumption 2, we can simply write it as $\Sigma=\left[\gamma\left(h_{i j}\right)\right]_{i, j=1}^{p}$, where $h_{i j}=\left\|s_{i}-s_{j}\right\|_{2}$ is the Euclidean distance between sites $s_{i}$ and $s_{j}$. Let $K_{T}(h, w)$ denote a tapering function, which is an isotropic autocorrelation function when $0<h<w$ and 0 when $h \geq w$ for a given threshold $w>0$. We use a simple tapering function from Wendland [41],

$$
K_{T}(h, \omega)=\left[(1-h / w)_{+}\right]^{2}
$$

where $x_{+}=\max (x, 0)$ meaning that the correlation is 0 when the lag distance $h$ is greater than the threshold distance $w$. Let $\mathbf{K}(w)=\left[K_{T}\left(h_{i i^{\prime}}, w\right)\right]_{i, i^{\prime}=1}^{p}$ denote the $p \times p$ tapering matrix. Then a tapered covariance of $\Sigma$ is defined as $\Sigma_{T}=$ $\Sigma \circ \mathbf{K}(w)$, where $\circ$ is the Schur product (i.e. elementwise product). By the properties of the Schur product (Horn and Johnson [22], chap. 5), the tapered covariance matrix would keep the positive definiteness thus it is still a valid covariance matrix. When $p$ is large, we approximate the penalized log-likelihood (3.1) by replacing $\Sigma$ with $\Sigma_{T}$ and obtain a covariance tapered penalized loglikelihood: 


$$
\begin{aligned}
& Q_{T}(\boldsymbol{\theta}, \boldsymbol{\beta} ; \mathbf{Z}) \\
= & -\frac{n p}{2} \log (2 \pi)-\frac{1}{2} \log \left|\dot{\Sigma}_{T}\right|-\frac{1}{2}(\mathbf{Z}-\boldsymbol{X} \boldsymbol{\beta})^{T} \dot{\Sigma}_{T}^{-1}(\mathbf{Z}-\boldsymbol{X} \boldsymbol{\beta})-n \sum_{j=1}^{p} P_{\lambda}\left(\left|\beta_{j}\right|\right) \\
= & C_{n, p}-\frac{n-1}{2} \log \left|\Sigma_{T}\right|-\frac{1}{2}(\mathbf{Z}-\boldsymbol{X} \boldsymbol{\beta})^{T} \operatorname{diag}_{n-1}\left(\Sigma_{T}^{-1}\right)\left(\tilde{I}_{n-1, p}+\tilde{J}_{n-1, p}\right)(\mathbf{Z}-\boldsymbol{X} \boldsymbol{\beta}) \\
& -n \sum_{j=1}^{p} P_{\lambda}\left(\left|\beta_{j}\right|\right)
\end{aligned}
$$

where $C_{n, p}=-\frac{(n-1) p}{2} \log \pi+\frac{p}{2} \log n$.

We keep all the notations the same as in (3.1), except that $\Sigma$ is replaced by $\Sigma_{T}$. We follow the one-step PMLE procedure. Let $\hat{\boldsymbol{\beta}}_{T, \text { ose }}=\hat{\boldsymbol{\Delta}}_{T, \text { ose }}$ and $\hat{\boldsymbol{\theta}}_{T, \text { ose }}$ be the one-step PMLE with tapered covariance $\left(\mathrm{PMLE}_{T, \text { ose }}\right)$. Next, we prove the consistency of PMLE $\mathrm{PM}_{T, \text { ose }}$. Let $\gamma_{k}(\boldsymbol{\theta}, h)=\frac{\partial \gamma(\boldsymbol{\theta}, h)}{\partial \theta_{k}}(\boldsymbol{\theta})$ and $\gamma_{j k}(\boldsymbol{\theta}, h)=\frac{\partial^{2} \gamma(\boldsymbol{\theta}, h)}{\partial \theta_{k} \theta_{j}}$. Two additional assumptions are made here for regularization.

Assumption 11. Assume $0<\inf _{p}\left\{\frac{w_{p}}{p^{\delta}}\right\}<\sup _{p}\left\{\frac{w_{p}}{p^{\delta}}\right\}<\infty$, where $w_{p}$ is the threshold distance in the tapering function for some $\delta>0$.

Assumption 12. Let $d(d \geq 1)$ be the dimension of the domain, i.e. $D \subset \mathbb{R}^{d}$. Assume for all $\boldsymbol{\theta} \in \Xi$ and $1 \leq k, j \leq q$, we have $\gamma(\boldsymbol{\theta}, h), \gamma_{k}(\boldsymbol{\theta}, h), \gamma_{j k}(\boldsymbol{\theta}, h)$ belong to the function space $£$, where $£=\left\{f(h): \int_{0}^{\infty} h^{d} f(h) d h<\infty\right\}$.

Let $\Sigma$ be the covariance matrix and $\Sigma_{T}$ be the tapered covariance matrix. $\Sigma_{k, T}=\frac{\partial \Sigma_{T}}{\partial \theta_{k}}$ and $\Sigma_{j k, T}=\frac{\partial^{2} \Sigma_{T}}{\partial \theta_{j} \partial \theta_{k}}$. By using the tapering function (3.2), we have the following result for $\mathrm{PMLE}_{T, \text { ose }}$.

Similar to Theorem 3.1, since the initial estimates have good properties, the one-step PMLE with tapering $\left(\mathrm{PMLE}_{T, \text { ose }}\right)$ also has good properties hence we have the following theorem:

Theorem 3.2. Assume conditions 2-12 hold. Assume $\boldsymbol{\beta}_{0}=\left(\boldsymbol{\beta}_{1,0}^{T}, \boldsymbol{\beta}_{2,0}^{T}\right)^{T}$, where $\boldsymbol{\beta}_{1,0} \in \mathbb{R}^{s}$ is non-zero component, $\boldsymbol{\beta}_{2,0}=\boldsymbol{O}_{(p-s) \times 1}$ is the zero component of $\boldsymbol{\beta}_{0}$ with $\frac{s}{n} \rightarrow 0, \frac{p}{n} \rightarrow C$ with $0<C \leq \infty$ as $n, p, s \rightarrow \infty$. The one-step PMLE estimates of (3.3) from Algorithm in Section 3.1 (PMLE $\left.E_{T, \text { ose }}\right)$ is $\hat{\boldsymbol{\eta}}_{T, \text { ose }}=$ $\left(\hat{\boldsymbol{\beta}}_{T, \text { ose }}, \hat{\boldsymbol{\theta}}_{T, \text { ose }}\right)$ with $\hat{\boldsymbol{\beta}}=\left(\hat{\boldsymbol{\beta}}_{1, T, \text { ose }}^{T}, \hat{\boldsymbol{\beta}}_{2, T, \text { ose }}^{T}\right)^{T}$ and $\hat{\boldsymbol{\beta}}_{1, T, \text { ose }}$ is a sub-vector of $\hat{\boldsymbol{\beta}}_{T, \text { ose }}$ formed by non-zero components in supp $\left(\boldsymbol{\beta}_{0}\right)$. Then $\hat{\boldsymbol{\eta}}_{T}$ satisfy:

(a) (consistency) $\left\|\hat{\boldsymbol{\theta}}_{T, \text { ose }}-\boldsymbol{\theta}_{0}\right\|_{2}=O_{p}\left(\frac{1}{\sqrt{n p}}\right)$ and $\left\|\hat{\boldsymbol{\beta}}_{T, \text { ose }}-\boldsymbol{\beta}_{0}\right\|_{2}=O_{P}\left(\sqrt{\frac{s}{n}}\right)$.

(b) (sparsity) $\hat{\boldsymbol{\beta}}_{2, T, \text { ose }}=0$ with probability tending to 1 as $n \rightarrow \infty$.

Proof. See Appendix A.3.

\subsection{The penalized maximum likelihood estimation LDA (PMLE-LDA) classifier}

Now we can develop the PMLE-LDA classifier in this section. No matter using $\mathrm{PMLE}_{\text {ose }}$ or $\mathrm{PMLE}_{T, \text { ose }}$, we obtain the consistent estimates for $\boldsymbol{\Delta}$ and $\boldsymbol{\theta}$ denoted 
by $\hat{\boldsymbol{\Delta}}$ and $\hat{\boldsymbol{\theta}}$. The estimation of $\boldsymbol{\mu}_{1}$ and $\boldsymbol{\mu}_{2}$ are $\hat{\boldsymbol{\mu}}_{1}=\overline{\boldsymbol{Y}}-\tau_{2} \hat{\boldsymbol{\Delta}}$ and $\hat{\boldsymbol{\mu}}_{2}=\overline{\boldsymbol{Y}}+\tau_{1} \hat{\boldsymbol{\Delta}}$. Besides, we have estimated covariance $\hat{\Sigma}=\Sigma(\hat{\boldsymbol{\theta}})$, where the $(i, j)$ th element of $\hat{\Sigma}$ is:

$$
\hat{\sigma}_{i j}=\gamma\left(\left|s_{j}-s_{i}\right| ; \hat{\boldsymbol{\theta}}\right)
$$

When $p>n$, the error accumulated in estimate of each $\hat{\sigma}_{i j}$ may also cause problems in classification (see Bickel and Levina [4] and Shao et al. [38]). For regularization of the covariance matrix, we use the tapered covariance matrix in classification function. Specifically, we define $\tilde{\Sigma}=\Sigma_{T}(\hat{\boldsymbol{\theta}})=\Sigma(\hat{\boldsymbol{\theta}}) \circ \mathbf{K}(w)$, where $\mathbf{K}(w)$ is defined in Section 3.1.2. We then replace $\boldsymbol{\mu}_{1}, \boldsymbol{\mu}_{2}, \Sigma$ in LDA (1.7) by $\hat{\boldsymbol{\mu}}_{1}, \hat{\boldsymbol{\mu}}_{2}$ and $\tilde{\Sigma}$ for classification. Then the PMLE-LDA function is:

$$
\hat{\delta}_{P L D A}(\boldsymbol{X})=\left(\boldsymbol{X}-\overline{\boldsymbol{Y}}-\frac{n_{1}-n_{2}}{2 n} \hat{\boldsymbol{\Delta}}\right)^{T} \tilde{\Sigma}^{-1} \hat{\boldsymbol{\Delta}}
$$

where $\overline{\boldsymbol{Y}}=\frac{1}{n} \sum_{k=1}^{2} \sum_{i=1}^{n_{k}} \boldsymbol{Y}_{k i}$.

The conditional misclassification rate for class 1 and class 2 are defined by (1.2) and (1.4) with $\hat{\Sigma}$ replaced with $\tilde{\Sigma}$. Similarly we have the overall misclassification rate defined in (1.6).

We need more assumptions for the covariance function $\gamma(h ; \boldsymbol{\theta})$ in Theorem 3.3.

Assumption 13. Let $d(d \geq 1)$ be the dimension of the domain $D$, i.e. $D \subset R^{d}$. Assume $\int_{1}^{\infty} h^{d} \gamma(h ; \boldsymbol{\theta}) d h<\infty$ and $\int_{0}^{1} h^{d-1} \gamma(h ; \boldsymbol{\theta}) d h<\infty$ for $\boldsymbol{\theta} \in \Xi$.

This requires that when $h \rightarrow \infty, \gamma(h ; \boldsymbol{\theta}) \sim h^{x}$ with $x<-(d+1)$ and when $h \rightarrow 0, \gamma(h ; \boldsymbol{\theta}) \sim h^{x}$ with $x>-d$.

Assumption 14. Assume there exist a constant $M$ such that for any $h \geq 0$ and $\boldsymbol{\theta} \in \Xi,\left\|\frac{\partial \gamma(h ; \boldsymbol{\theta})}{\partial \boldsymbol{\theta}}\right\|_{2} \leq M$.

Theorem 3.3. Assume $\hat{\boldsymbol{\theta}}, \hat{\boldsymbol{\Delta}}$ in (3.5) are estimated from Theorem 3.1 or 3.2. Suppose Assumptions 2-3 and Assumptions 13-14 hold. Assume $\frac{s}{n} \rightarrow 0, \frac{p}{n} \rightarrow C$ with $0<C \leq \infty, C_{p} \rightarrow C_{0}$ with $0 \leq C_{0} \leq \infty, \frac{C_{p}}{\sqrt{s / n}} \rightarrow 0$. Also, assume $w=O\left((\sqrt{n p})^{\frac{\alpha}{d}}\right)$ with $0<\alpha<1$, and $w^{-1}=O\left(p^{-\delta}\right)$ with some $\delta>0$, where $d$ is the dimension of the domain. Then the classification error rate of $\hat{\delta}_{P L D A}$ is asymptotically sub-optimal, i.e. $W(\hat{\delta}) \stackrel{P}{\rightarrow} 1-\Phi\left(\frac{\sqrt{C_{0}}}{2}\right)$. Moreover,

(1) If $C_{p} \rightarrow C_{0}<\infty, W(\hat{\delta})$ is asymptotically optimal, i.e. $\frac{W(\hat{\delta})}{W_{O P T}} \stackrel{P}{\rightarrow} 1$;

(2) If $C_{p} \rightarrow \infty$ and $C_{p} \kappa_{n, p} \rightarrow 0, W(\hat{\delta})$ is asymptotically optimal, i.e. $\frac{W(\hat{\delta})}{W_{O P T}} \stackrel{P}{\rightarrow}$ 1 , where $\kappa_{n, p}=\max \left(\frac{w^{d}}{\sqrt{n p}}, \frac{1}{w}, \sqrt{\frac{s}{n}}\right)$.

Proof. See Appendix A.4.

Since $W_{O P T}=1-\Phi\left(\frac{\sqrt{C_{p}}}{2}\right) \rightarrow 1-\Phi\left(\frac{\sqrt{C_{0}}}{2}\right)$, Theorem 3.3 shows that with moderate conditions, the error rate of the proposed classifier goes to the unique limit $W_{O P T}$. Moreover, if $C_{p} \rightarrow C_{0}<\infty$ or $C_{p}$ goes to $\infty$ with a moderate rate, then $W(\hat{\delta})$ goes to $1-\Phi\left(\frac{\sqrt{C_{0}}}{2}\right)$ with the same rate as $W_{O P T}$. 


\begin{tabular}{|c|c|c|c||c|c|c|c||c|c|c|c|}
\hline 4 & 8 & 12 & 16 & 1 & 1 & 0 & 0 & 0 & 0 & 0 & 0 \\
\hline 3 & 7 & 11 & 15 & 1 & 1 & 0 & 0 & 0 & 0 & 0 & 0 \\
\hline 2 & 6 & 10 & 14 & 1 & 1 & 1 & 0 & 0 & 0 & 0 & 0 \\
\hline 1 & 5 & 9 & 13 & 1 & 1 & 1 & 0 & 0 & 0 & 0 & 0 \\
\hline
\end{tabular}

FIG 1. Two dimensional domain example. Left: $2 D$ domain with $p=4 \times 4$; middle: $\boldsymbol{\mu}_{1}$; right: $\boldsymbol{\mu}_{2}$.

\section{Simulation study}

We conduct extensive simulation study to evaluate the performance of our proposed method compared to available generic procedures. Then we illustrate the methodology with real neuroimgaing data from ADNI.

Assume that the spatial domain of interest $D$ in $\mathbb{R}^{2}$ is a $u \times u$ square area. We can observe signal at each lattice. Then we have $p=u \times u$ features for classification. The mean effects of the signal for class $\mathcal{C}_{1}$ and $\mathcal{C}_{2}$ are $\boldsymbol{\mu}_{1}$ and $\boldsymbol{\mu}_{2}$. We assume that $\boldsymbol{\mu}_{1}=\left(\mathbf{1}_{10}, \mathbf{0}_{p-10}\right)$ and $\boldsymbol{\mu}_{2}=\mathbf{0}_{p}$, where $\mathbf{1}_{k}$ is a $k$ dimension vector with all the elements equal to 1 and $\mathbf{0}_{k}$ is a $k$ dimension vector with all the elements equal to 0 . For example, if $u=4$ (hence $p=16$ ), then the corresponding spatial domain $D$, and the mean structure of $\boldsymbol{\mu}_{1}$ and $\boldsymbol{\mu}_{2}$ are shown as in Figure 1. In the simulation setting, we construct various simulation scenarios by letting $u=6,20$, and 35, making $p=36,400,1225$ respectively.

For the spatial covariance, we generate the error terms from stationary and isotropic Gaussian process with zero mean. A widely used spatial covariance function Matérn covariance was defined in (2.6). We use a special case of Matérn covariance function when $\nu=\frac{1}{2}$, which is the exponential covariance function. In the simulation, we set the variance scale as $\sigma^{2}=1$, the nugget effect as $c=0.2$ and the range parameter as $r=1,2, \ldots, 8,9$. Larger value of $r$ means longer range spatial dependency. Let $h$ be the Euclidean distance between two sites on the domain $D$. Specifically, on the domain $D \in \mathbb{R}^{2}$, the distance between site $i$ with coordinate $s_{i}=\left(x_{i}, y_{i}\right)$ and site $j$ with coordinate $s_{j}=\left(x_{j}, y_{j}\right)$ is $h_{i j}=\sqrt{\left(x_{i}-x_{j}\right)^{2}+\left(y_{i}-y_{j}\right)^{2}}$.

We generate 100 groups of training sets with $n_{1}=n_{2}=30$ according to different setting of $\boldsymbol{\mu}_{1}, \boldsymbol{\mu}_{2}$ and $\Sigma\left(\boldsymbol{\theta}_{0}\right)$. For each training set, we estimate the parameters $\boldsymbol{\mu}_{1}, \boldsymbol{\mu}_{2}$ and $\boldsymbol{\theta}_{0}$ by MLE, tapered MLE, PMLE and tapered PMLE. We also generate 100 groups of testing data sets with $n_{1}=n_{2}=100$ to test the classification performance. The average classification error rate was calculated from the 100 groups of testing data sets.

We name our classification method proposed in this paper as PMLE-LDA. For each choice of $p$, we compare the classification performance of PMLE-LDA with MLE-LDA, PREG-LDA, FAIR (Feature Annealed Independence Rule) and NB (Naive Bayes) and RPEC(Random-projection ensemble classification). 
More specifically, PMLE-LDA is the classifier defined in (3.5); MLE-LDA uses $\hat{\boldsymbol{\mu}}_{1 M L E}, \hat{\boldsymbol{\mu}}_{2 M L E}$ and $\Sigma\left(\hat{\boldsymbol{\theta}}_{M L E}\right)$ in LDA function for classification; PREG-LDA uses $\hat{\boldsymbol{\Delta}}=\hat{\boldsymbol{\beta}}^{(0)}$ and $\hat{\boldsymbol{\theta}}=\hat{\boldsymbol{\theta}}^{(0)}$ in LDA function, where $\hat{\boldsymbol{\beta}}^{(0)}$ and $\hat{\boldsymbol{\theta}}^{(0)}$ are estimated in the first and second step in the procedure in Section 3.1. NB [3] uses sample mean $\hat{\boldsymbol{\mu}}_{1}, \hat{\boldsymbol{\mu}}_{2}$ and diagonal of sample covariance $\hat{\Sigma}$ in LDA. This method is also known as independent rule(IR). FAIR [15] assumes independence between variables and utilizes t-test for variable selection in NB. RPEC [11] is a very general method which is not designed for a specific classifier (e.g. LDA). It uses random projections to project the feature vectors from any classifier into a lower dimensional space. To compare with the truth, we uses TRUE to denote that true mean $\boldsymbol{\mu}_{1}, \boldsymbol{\mu}_{2}$ and true covariance $\Sigma\left(\boldsymbol{\theta}_{0}\right)$ are used in LDA for classification.

We recall the basic differences of these methods as follows. RPEC, FAIR and NB are the classification methods without considering spatial dependency, while MLE-LDA, PREG-LDA and PMLE-LDA are methods considering spatial dependency. MLE and NB are two methods without feature selection, while PREG-LDA, PMLE-LDA and FAIR are classification methods with feature selection. Moreover, PREG-LDA selects features by penalized regression without considering spatial dependency, while PMLE-LDA selects features by penalized maximum likelihood estimation with spatial dependency incorporated.

We also compared our method with four more methods, which are $l_{1}$-logistic regression, $l_{1}$-FDA, DSDA (Direct sparse discriminant analysis) and $\mathrm{CATCH}$ (Covariate-adjusted tensor classification in high-dimensions). $l_{1}$-logistic regression is one of the most basic and popular methods to solve a classification problem. $l_{1}$-LDA is proposed for penalizing the discriminant vectors in Fisher's discriminant problem (Witten and Tibshirani [42]). DSDA generalizes classical LDA and formulates high-dimensional LDA into a penalized least squares problem (Mai, Zou and Yuan [30]). CATCH takes advantage of the tensor structure to significantly reduce the number of parameters and hence alleviate computation complexity (Pan, Mai and Zhang [35]).

The classification performance of all the methods is shown in Table 1. $r=1$, $r=5$ and $r=9$ means weak, moderate and strong spatial dependence respectively. Among all the methods, PMLE-LDA outperforms all the others. We have the following conclusions. First, when spatial dependency is weak $(r=1)$, all the methods with or without spatial dependency do not have much difference. But when spatial dependency is strong $(r=9)$, the methods with spatial dependency (MLE-LDA, PREG-LDA, PMLE-LDA) outperform the methods without spatial dependency (FAIR, NB, RPEC, $l_{1}$-logistic, $l_{1}$-LDA, DSDA and $\mathrm{CATCH})$. Second, when the number of feature is small $(p=36)$, the methods with or without feature selection have similar performance. But when number of feature is large ( $p=400$ and $p=1225)$, the methods with feature selection outperforms the methods without feature selection. PREG-LDA and PMLE-LDA outperforms MLE-LDA. FAIR outperforms NB. Third, CATCH outperforms $l_{1}$ logistic, $l_{1}$-LDA and DSDA, because it honors the tensor structure and preserves more information. But CATCH does not consider the spatial dependency, so the performance is not as good as PMLE-LDA. In the end, PMLE-LDA outperforms 
TABLE 1

Comparisons of classification accuracy rate.

\begin{tabular}{|c|c|c|c|c|c|c|c|c|c|c|c|}
\hline & TRUE & MLE & PREG & PMLE & FAIR & NB & RPEC & $l_{1}$-logistic & $l_{1}$-LDA & DSDA & CATCH \\
\hline & $p=36$ & & & & & & & & & & \\
\hline$r=1$ & $0.88(0.02)$ & $0.84(0.03)$ & $0.84(0.04)$ & $0.84(0.05)$ & $0.81(0.04)$ & $0.84(0.04)$ & $0.82(0.03)$ & $0.81(0.04)$ & $0.83(0.03)$ & $0.80(0.05)$ & $0.83(0.04)$ \\
\hline$r=5$ & $0.91(0.02)$ & $0.88(0.02)$ & $0.88(0.02)$ & $0.88(0.04)$ & $0.72(0.05)$ & $0.75(0.05)$ & $0.85(0.03)$ & $0.83(0.04)$ & $0.75(0.06)$ & $0.83(0.04)$ & $0.84(0.04)$ \\
\hline \multirow[t]{2}{*}{$r=9$} & $0.94(0.02)$ & $0.91(0.02)$ & $0.92(0.02)$ & $0.92(0.02)$ & $0.71(0.05)$ & $0.75(0.05)$ & $0.88(0.03)$ & $0.87(0.03)$ & $0.75(0.08)$ & $0.86(0.04)$ & $0.87(0.03)$ \\
\hline & $p=400$ & & & & & & & & & & \\
\hline$r=1$ & $0.92(0.02)$ & $0.74(0$. & 0.84 & $0.82(1$ & 0.83 & $0.74(0$ & 0.73 & & 0.7 & 0.79 & $0.83(0.04)$ \\
\hline$r=5$ & $0.95(0.01)$ & $0.81(0.03)$ & $0.90(0.05)$ & $0.92(0.03)$ & $0.74(0.04)$ & $0.60(0.05)$ & $0.68(0.04)$ & $0.77(0.05)$ & $0.59(0.06)$ & $0.79(0.05)$ & $0.78(0.06)$ \\
\hline \multirow[t]{2}{*}{$r=9$} & $0.97(0.01)$ & $0.86(0.02)$ & $0.93(0.04)$ & $0.96(0.02)$ & $0.72(0.05)$ & $0.58(0.05)$ & $0.71(0.04)$ & $0.82(0.05)$ & $0.56(0.06)$ & $0.83(0.05)$ & $0.82(0.04)$ \\
\hline & $p=1225$ & & & & & & & & & & \\
\hline$r=1$ & $0.92(0.02)$ & $0.65(0$. & & & 0.83 & $0.66(0$ & 0.65 & & & & $0.81(0.04)$ \\
\hline$r=5$ & $0.95(0.02)$ & $0.72(0.03)$ & $0.86(0.07)$ & $0.90(0.04)$ & $0.74(0.05)$ & $0.56(0.05)$ & $0.58(0.04)$ & $0.73(0.05)$ & $0.53(0.04)$ & $0.74(0.05)$ & $0.73(0.04)$ \\
\hline$r=9$ & $0.97(0.01)$ & $0.76(0.03)$ & $0.89(0.07)$ & $0.93(0.03)$ & $0.72(0.05)$ & $0.54(0.05)$ & $0.58(0.04)$ & $0.76(0.05)$ & $0.52(0.03)$ & $0.78(0.05)$ & $0.76(0.05)$ \\
\hline
\end{tabular}

TABLE 2

Comparisons of parameter estimation.

\begin{tabular}{cc|c|cc|cc|cc}
\hline & & & \multicolumn{2}{|c}{$p=36$} & \multicolumn{2}{c}{$p=400$} & \multicolumn{2}{c}{$p=1225$} \\
\hline & & TRUE & MLE & PMLE & MLE & PMLE & MLE & PMLE \\
\hline$r=1$ & $\mathrm{r}$ & 1 & $1.01(0.16)$ & $1.04(0.16)$ & $1(0.05)$ & $1(0.05)$ & $1.00(0.02)$ & $1.00(0.01)$ \\
& $\mathrm{c}$ & 0.2 & $0.19(0.12)$ & $0.19(0.12)$ & $0.2(0.04)$ & $0.19(0.03)$ & $0.20(0.01)$ & $0.20(0.02)$ \\
& $\sigma$ & 1 & $0.97(0.03)$ & $1(0.04)$ & $0.97(0.01)$ & $1(0.01)$ & $0.97(0.01)$ & $1.00(0.01)$ \\
\hline$r=5$ & $\mathrm{r}$ & 5 & $5.08(0.75)$ & $5.09(0.75)$ & $5.03(0.27)$ & $5.04(0.27)$ & $4.99(0.17)$ & $5.00(0.17)$ \\
& $\mathrm{c}$ & 0.2 & $0.2(0.03)$ & $0.2(0.03)$ & $0.2(0.01)$ & $0.2(0.01)$ & $0.20(0.004)$ & $0.20(0.004)$ \\
& $\sigma$ & 1 & $0.97(0.08)$ & $1.01(0.08)$ & $0.97(0.03)$ & $1.01(0.04)$ & $0.97(0.02)$ & $1.00(0.02)$ \\
\hline$r=9$ & $\mathrm{r}$ & 9 & $9.17(1.58)$ & $9.1(1.57)$ & $9.1(0.67)$ & $9.12(0.69)$ & $8.96(0.45)$ & $8.97(0.45)$ \\
& $\mathrm{c}$ & 0.2 & $0.2(0.02)$ & $0.2(0.02)$ & $0.2(0.01)$ & $0.2(0.01)$ & $0.20(0.01)$ & $0.20(0.01)$ \\
& $\sigma$ & 1 & $0.97(0.1)$ & $1.01(0.1)$ & $0.97(0.05)$ & $1.01(0.05)$ & $0.96(0.03)$ & $1.00(0.03)$ \\
\hline
\end{tabular}

PREG-LDA, which implies the selection procedure considering spatial dependency outperforms feature selection without considering spatial dependency.

The parameter estimation results are shown in Table 2. It shows the parameters are all consistently estimated. We also compared the average number of variables selected from PMLE-LDA, PREG-LDA and FAIR in Table 3. The tunning parameter $\lambda$ in PREG-LDA and PMLE-LDA is selected by 10 fold cross validation by minimizing the classification error rate. Table 3 shows that FAIR tends to select the fewest features. PMLE tends to select more features than PREG. But when spatial dependency is strong, PMLE produces smaller variance for feature selection and thus smaller misclassification rate.

Additionally, we investigate the performance of classification, parameter estimation and feature selection of tapered MLE-LDA, tapered PMLE-LDA and tapered PREG-LDA. Note that the tapering technique is applied in parameter estimation. The performances of classification and feature selection are similar with the ones without tapering (see Tables 1 and 3). However, the tapering technique estimates a larger range parameter $r$ when the spatial dependency is strong. This is consistent with the characteristic of tapering technique. To save space, these tables are omitted here but are available in $\mathrm{Li}$ [25].

Finally, we investigate the simulation results when covariance is mis-specified. More specifically, we use Gaussian covariance function defined in Section 2.1 (i.e. Matérn covariance when $\nu \rightarrow \infty)$ to generate the data. Then we use exponential covariance function (i.e. Matérn covariance when $\nu=\frac{1}{2}$ ) to estimate the structure and complete classification. Both of them are Matérn covariance with different smoothness parameters. We generate the data using Gaussian 
TABLE 3

Comparisons of number of selected features.

\begin{tabular}{|c|c|c|c|c|c|c|}
\hline & \multicolumn{2}{|c|}{ PMLE } & \multicolumn{2}{|c|}{ PREG } & \multicolumn{2}{|c|}{ FAIR } \\
\hline$p=36$ & selectedN & correctN & selectedN & correctN & selectedN & correctN \\
\hline$r=1$ & $20.77(6.21)$ & $9.47(1.71)$ & $18.92(7.03)$ & $9.81(0.8)$ & $6.45(3.98)$ & $5.48(2.36)$ \\
\hline$r=5$ & $19.97(5.51)$ & $9.51(1.52)$ & $19.97(7.06)$ & $10(0)$ & $3.23(1.48)$ & $3.08(1.47)$ \\
\hline$r=9$ & $19.8(5.04)$ & $9.72(1.07)$ & $19.45(7.57)$ & $10(0)$ & $2.81(1.38)$ & $2.68(1.34)$ \\
\hline \multicolumn{7}{|l|}{$p=400$} \\
\hline$r=1$ & $84.8(55.3)$ & $9.2(1.5)$ & $42(55.2)$ & $9.2(1.4)$ & $20.8(15.1)$ & $7.2(2.1)$ \\
\hline$r=5$ & $73.7(32.2)$ & $9.8(0.6)$ & $50.7(73.3)$ & $9.5(1.2)$ & $11.3(10.6)$ & $5.3(2.8)$ \\
\hline$r=9$ & $52.5(21.9)$ & $9.9(0.5)$ & $63.7(98.5)$ & $9.7(1.1)$ & $6.8(7.6)$ & $4.1(2.7)$ \\
\hline \multicolumn{7}{|l|}{$p=1225$} \\
\hline$r=1$ & $181.3(205.3)$ & $7.85(2.83)$ & $37.48(59.38)$ & $8.59(1.85)$ & $31.07(22.28)$ & $6.96(1.73)$ \\
\hline$r=5$ & $174.5(126.3)$ & $9.48(1.53)$ & 76.88(191.66) & $8.89(1.84)$ & $26.15(20.55)$ & $6.42(2.83)$ \\
\hline$r=9$ & $115.1(58.9)$ & $9.81(0.88)$ & $79.21(192.62)$ & $9.1(1.81)$ & $15.28(14.02)$ & $5.28(3.41)$ \\
\hline
\end{tabular}

TABLE 4

Comparisons of classification accuracy rate when covariance is mis-specified.

\begin{tabular}{c|ccccccc}
\hline & TRUE & MLE & PREG & PMLE & FAIR & NB & RPEC \\
\hline & $p=36$ & & & & & \\
\hline$r=1$ & $0.89(0.02)$ & $0.84(0.03)$ & $0.84(0.04)$ & $0.84(30.05)$ & $0.83(0.05)$ & $0.85(0.05)$ & $0.83(0.03)$ \\
$r=5$ & $0.94(0.02)$ & $0.92(0.02)$ & $0.92(0.02)$ & $0.93(0.03)$ & $0.71(0.05)$ & $0.74(0.05)$ & $0.90(0.03)$ \\
$r=9$ & $0.97(0.01)$ & $0.96(0.01)$ & $0.95(0.02)$ & $0.96(0.02)$ & $0.71(0.05)$ & $0.74(0.05)$ & $0.94(0.02)$ \\
\hline & $p=400$ & & & & & \\
\hline$r=1$ & $0.91(0.02)$ & $0.73(0.03)$ & $0.84(0.05)$ & $0.83(0.05)$ & $0.85(0.05)$ & $0.75(0.03)$ & $0.74(0.04)$ \\
$r=5$ & $0.98(0.01)$ & $0.88(0.02)$ & $0.94(0.03)$ & $0.97(0.02)$ & $0.73(0.04)$ & $0.59(0.04)$ & $0.67(0.04)$ \\
$r=9$ & $0.99(0.01)$ & $0.94(0.02)$ & $0.97(0.02)$ & $0.98(0.01)$ & $0.70(0.06)$ & $0.56(0.04)$ & $0.75(0.05)$ \\
\hline & $p=1225$ & & & & & \\
\hline$r=1$ & $0.91(0.02)$ & $0.64(0.04)$ & $0.82(0.05)$ & $0.77(0.06)$ & $0.83(0.05)$ & $0.67(0.05)$ & $0.65(0.04)$ \\
$r=5$ & $0.98(0.01)$ & $0.78(0.03)$ & $0.90(0.07)$ & $0.95(0.02)$ & $0.73(0.05)$ & $0.54(0.05)$ & $0.56(0.04)$ \\
$r=9$ & $0.99(0.01)$ & $0.85(0.03)$ & $0.92(0.07)$ & $0.96(0.03)$ & $0.71(0.06)$ & $0.53(0.06)$ & $0.57(0.04)$ \\
\hline
\end{tabular}

covariance function with $\sigma^{2}=1, c=0.2$, and $r=1,2, \ldots, 9$. Table 4 shows the classification performance if the covariance are misspecified. It shows that with misspecified covariance, the PMLE-LDA classification method has the best performance, even when the spatial dependency is strong $(r=5$ and $r=9)$. Therefore, the proposed method is robust to the mis-specification of covariance.

\section{Real data application}

Alzheimer's disease (AD) is a neuro-degenerative disease and the most common form of dementia, affecting many millions around the world. Classification of $\mathrm{AD}$ patients is a crucial task in dementia research. To apply our classification method, we obtain the data from the Alzheimer's disease Neuroimaging Initiative (ADNI) database (http://www.loni.ucla.edu/ADNI), which was launched in 2004. ADNI aims to improve clinical trials for prevention and treatment of Alzheimer's disease (AD). With the interest of promoting consistency in data analysis, the ADNI Core has created standardized analysis sets of the structured MRI scans comprising only image data that have passed quality control (QC) assessments. The assessments were conducted at the Aging 
TABLE 5

Subjects characteristics.

\begin{tabular}{llll}
\hline & AD & NL & p-value \\
\hline$n$ & 187 & 227 & \\
Age (Mean $\pm \mathrm{sd})$ & $75.28 \pm 7.55$ & $75.80 \pm 4.98$ & 0.4168 \\
Gender $(\mathrm{F} / \mathrm{M})$ & $88 / 99$ & $110 / 117$ & 0.813 \\
MMSE (Mean $\pm \mathrm{sd})$ & $23.28 \pm 2.04$ & $29.11 \pm 1.00$ & $<1 e-15$ \\
\hline Key: AD, subjects with Alzheimer's disease; NL, healthy subjects; \\
Age, baseline age; MMSE, baseline Mini-Mental State Examination.
\end{tabular}

and Dementia Imaging Research laboratory at the Mayo Clinic (Jack et al. [24]). In this study, we used T1-weighted MRI images from the collection of standardized datasets. The description of the standardized MRI imaging from ADNI can be found in http://adni.loni.usc.edu/methods/mri-analysis/ adni-standardized-data/ and Wyman et al. [44].

According to Jack et al. [24], the images were generated using magnetization prepared rapid gradient echo (MPRAGE) or equivalent protocols with varying resolutions (typically $1.0 \times 1.0 \mathrm{~mm}$ in plane spatial resolution and $1.2 \mathrm{~mm}$ thick sagittal slices with $256 \times 256 \times 166$ voxels). The images were then pre-processed according to a number of steps detailed in Jack et al. [24] and http://adni.loni. usc.edu/methods/mri-analysis/mri-pre-processing/, which corrected gradient non-linearity, intensity inhomogeneity and phantom-based distortion. In addition, the pre-processed imaging were processed by FreeSurfer for cortical reconstruction and volumetric segmentation by Center for Imaging of Neurodegnerative Diseases, UCSF.

In this paper, we obtain images from ADNI-1 subjects obtained using $1.5 \mathrm{~T}$ scanners at screening visits. We use the first time point if there are multiple images of the same subject acquired at different times. 187 subjects diagnosed as Alzheimer's disease at screening visits and 227 healthy subjects at screening visits are contained in this study. The total number of subjects is 414. Details of the subjects can be found in Table 5. The authors used ADNI data in their previous research work (Zhang et al. [46], Li et al. [28]). Please refer these papers for other information about the data.

After retrieving the pre-processed imaging data from ADNI, an $\mathrm{R}$ package ANTsR is applied for imaging registration. Then we use "3dresample" command by AFNI software (Cox [13]) to adjust the resolution and reduce the total number of voxels in the images to $18 \times 22 \times 18$ voxels. Take $x$ axis and $y$ axis for horizontal plane, $x$ axis and $z$ axis for coronal plane and $y$ axis and $z$ axis for sagittal plane.

After removing the voxels with zero signal for most of the subjects (more than 409 subjects), we have 1971 voxels left in use. The distance between each pair of voxels can be calculated by their coordinates. For example, there are two voxels $s_{1}, s_{2}$ with coordinate $s_{1}=\left(x_{1}, y_{1}, z_{1}\right)$ and $s_{2}=\left(x_{2}, y_{2}, z_{2}\right)$. Then the Euclidean distance between $s_{1}$ and $s_{2}$ is defined by: $d\left(s_{1}, s_{2}\right)=$ $\sqrt{\left(x_{1}-x_{2}\right)^{2}+\left(y_{1}-y_{2}\right)^{2}+\left(z_{1}-z_{2}\right)^{2}}$. Other distances can also be used in our method. 
TABLE 6

Subjects characteristics of training and testing set.

\begin{tabular}{lllll}
\hline & & training set & testing set & p-value \\
\hline $\mathrm{AD}$ & $n$ & 100 & 87 & \\
& Age $($ Mean $\pm \mathrm{sd})$ & $75.64 \pm 7.39$ & $74.85 \pm 7.75$ & 0.478 \\
& Gender $(\mathrm{F} / \mathrm{M})$ & $47 / 53$ & $41 / 46$ & 0.999 \\
& MMSE $($ Mean $\pm \mathrm{sd})$ & $23.22 \pm 2.08$ & $23.36 \pm 2.01$ & 0.649 \\
\hline $\mathrm{NL}$ & $n$ & 100 & 87 & \\
& Age (Mean $\pm \mathrm{sd})$ & $75.99 \pm 5.39$ & $75.34 \pm 4.56$ & 0.3723 \\
& Gender (F/M) & $42 / 58$ & $50 / 37$ & 0.05 \\
& MMSE (Mean $\pm \mathrm{sd})$ & $29.06 \pm 1.04$ & $29.09 \pm 1.01$ & 0.8307 \\
\hline
\end{tabular}

Key: AD, subjects with Alzheimer's disease; NL, healthy subjects;

Age, baseline age; MMSE, baseline Mini-Mental State Examination.

TABLE 7

Classification performance for voxel level MRI data. We split the data into Training sets (200 samples) and testing sets (174 samples) as described.

\begin{tabular}{ccccccc}
\hline & MLE & PREG & PMLE & FAIR & NB & RPEC \\
\hline Accuracy & 0.690 & 0.759 & 0.770 & 0.569 & 0.661 & 0.678 \\
No. of training err & 37 & 52 & 47 & 68 & 43 & 21 \\
No. of testing err & 54 & 42 & 40 & 75 & 59 & 56 \\
No. of selected voxels & 1971 & 26 & 4 & 16 & 1971 & - \\
\hline
\end{tabular}

We randomly sample 100 from the $187 \mathrm{AD}$ subjects and 100 from the 227 health subjects as the training set. Then there are $87 \mathrm{AD}$ subjects and 127 healthy subjects left. The testing set includes the $87 \mathrm{AD}$ subjects and a random sample of 87 from the 127 healthy subjects. Details of the subjects in the training and testing set are provided in Table 6 .

We assume the exponential correlation structure among voxels. Then we apply the PMLE-LDA method proposed in this research for classification. First, the parameter are estimated by PMLE: $r=61.66, c=0.954, \sigma^{2}=223.09$ and 5 voxels are selected for classification from training data. Then we plug-in the estimates into the classification function and obtain classification results on the testing data.

The classification accuracy rate of PMLE-LDA is listed in Table 7. We also list the classification accuracy from other methods. It shows the classification accuracy rate of our method is about $77.0 \%$, which is superior to other comparable methods (MLE-LDA: 69.0\%, PREG-LDA: $75.9 \%$, FAIR: $56.9 \%$, NB: $66.1 \%$ and RPEC: $67.8 \%)$.

To show the robustness of the proposed method, we repeat the above procedure for 100 times and calculated the average accuracy and standard deviation in Table 8. Although we know that MRI data is noisy in general and heterogeneous across subjects, our method still provide reasonably higher classification rates with small standard deviations.

\section{Conclusion and discussion}

The paper contains new developments for the classification problem of multivariate Gaussian variables with spatial structures. We generalize the classical 
TABLE 8

Average classification performance for voxel level MRI data. We repeated the training/testing procedure for 100 times.

\begin{tabular}{ccccccc}
\hline & MLE & PREG & PMLE & FAIR & NB & RPEC \\
\hline Accuracy & 0.704 & 0.704 & 0.703 & 0.577 & 0.691 & 0.695 \\
$(\mathrm{sd})$ & 0.036 & 0.035 & 0.035 & 0.054 & 0.034 & 0.032 \\
No. of selected voxels & 1971 & 1076.30 & 1043.35 & 6 & 1971 & - \\
$(\mathrm{sd})$ & - & 35.61 & 35.5 & 0.19 & - & - \\
\hline
\end{tabular}

LDA by assuming spatially dependent structures in the covariance and imposing sparsity on the feature difference. In particular, by using the Matérn covariance function, the $p \times p$ dimensional covariance is parameterized by only three univariate parameters. By utilizing the additional spatial location information and constructing the data-driven spatial correlation structure in the data, the new spatial LDA method is expected to be more efficient than other sparse LDA methods. Under this framework, we adopt Penalized Maximum Likelihood Estimation (PMLE) method to perform parameter estimation. Most importantly, we show in theory that the proposed method can not only provide consistent results of parameter estimation and feature selection, but also achieve an asymptotically optimal classifier for high dimensional data with spatial structures.

Brain imaging data are usually matrix-variate or tensor-variate observations. In this paper, we adopt one type of data arrangements that vectorize the tensor data into vectors and stack covariates along with the long vector to apply vector methods. This will inevitably increase the dimension of covariance matrix and thus lead to high computational burden regarding matrix operations. We can increase the speed by avoiding matrix inverse calculation (Bhattacharya, Chakraborty and Mallick [2]) or directly estimating sample covariance instead of using MLE. In the future, we plan to extend our current computing strategy to tensor methods (e.g. tensor LDA) that significantly reduce the number of parameters and hence alleviate computation complexity (Li and Zhang [27], Pan, Mai and Zhang [35]).

In general, nonparametric methods do not have the assumption on data distribution. It overcomes the limitation of parametric LDA, which can not perform well in non-Gaussian data. But intrinsically, these two types of methods are demanded to solve the same kind of problem, which is to resemble the changing pattern of brain regions. Mostly, they use average trend to denote the deterioration of brain function and use network structure to denote synergies of the functional connectivity. To model the average trend, typical methods such as kernel function, spline, wavelet and Fourier transformation are exploited to transform the original coordinates to a new space with better properties. To model the network structure, typical methods such as nonparametric graphical model, random matrices and Fourier transform of images are exploited to decompose the nodes into new clusters relevant information. However, one disadvantage of nonparametric method is that the results are not straightforward to interpret, as they are wrapped up in a "black box". In the future, we plan to extend our current method to nonparametric models by using Fourier transfor- 
mation. We will work more on how to include inverse transformation into the model so that the results are interpretable in the original space.

\section{Remarks on the assumptions}

\section{Remarks on Assumption 3:}

The first part of Assumption 3 is the same as that in Mardia and Marshall [31]. We now verify that the covariance matrix derived from Matérn covariance function satisfy the first part of Assumption 3. First, for symmetric matrix, we have

$$
\lambda_{\max }(\Sigma) \leq\left(\|\Sigma\|_{1}\right)^{1 / 2}\left(\|\Sigma\|_{\infty}\right)^{1 / 2}=\|\Sigma\|_{\infty}=\max _{i} \sum_{j}^{p} \gamma\left(h_{i j}\right)
$$

Using the same notation in the proof of Lemma 3 , for each $i$

$$
\begin{aligned}
\sum_{j=1}^{p} \gamma\left(h_{i j}\right) & \leq \sum_{m=0}^{\infty} \sum_{j \in B_{m}^{i}} r\left(h_{i j}\right) \leq K \rho \sum_{m=0}^{\infty} m^{d-1} \delta^{d} \max _{j \in B_{m}^{i}} r\left(h_{i j}\right) \\
& \leq K \rho \int_{0}^{\infty} h^{d-1} r(h) d h
\end{aligned}
$$

Recall that Matérn covariance function has the following expansion at $h=0$ :

$$
\gamma\left(h ; \sigma^{2}, c, \nu, r\right)=\sigma^{2}(1-c)\left(1-b_{1} h^{2 \nu}+b_{2} h^{2}+O\left(h^{2+2 \nu}\right)\right) \text { as } h \rightarrow 0
$$

where $b_{1}$ and $b_{2}$ are explicit constants depending only on $\nu$ and $r$. Thus for $\epsilon>0$,

$$
\int_{0}^{\epsilon} h^{d-1} \gamma(h) d h=O\left(\int_{0}^{\epsilon} h^{d-1} d h\right)=O\left(\epsilon^{d} / d\right) \rightarrow 0 \text { as } \epsilon \rightarrow 0
$$

Also, since $K_{\nu}(h) \propto e^{-h} h^{-\frac{1}{2}}\left(1+O\left(\frac{1}{h}\right)\right)$ as $h \rightarrow \infty$, there exist a constant $K$, for any $C$ sufficiently large, we have:

$$
\int_{C}^{\infty} h^{d-1} \gamma(h) d h \leq K \int_{0}^{\infty} h^{d-1+v-\frac{1}{2}} e^{-h} d h=\Gamma\left(d+v-\frac{1}{2}\right)<\infty
$$

Eqs. (7.2) and (7.3) lead to $\int_{0}^{\infty} h^{d-1} \gamma(h) d h<\infty$. Let $p \rightarrow \infty$ in (7.1), we have $\limsup _{p \rightarrow \infty} \lambda_{\max }(\Sigma)<\infty$ if $\Sigma$ is derived from Matérn covariance function.

Now consider the second part of Assumption 3. Assumption 1 assumes increasing domain framework. Bachoc and Furrer [1] showed that under Assumption 1 and some weak assumptions on the matrix covariance function, if the spectral density of the covariance function is positive, the smallest eigenvalue of the covariance matrix is asymptotically bounded away from zero. Most standard covariance function such as Matérn covariance function satisfy those assumptions hence satisfy the second part of Assumption 3. 


\section{Remarks on Assumption 4 and Assumption 5:}

Assumption 4 and Assumption 5 are the same as the assumptions in Mardia and Marshall [31]. $\left\|\Sigma_{k}\right\|_{F}=\sum_{i, j=1}^{p} \gamma_{k}^{2}\left(h_{i j} ; \boldsymbol{\theta}\right)$, where $\gamma_{k}\left(h_{i j} ; \boldsymbol{\theta}\right)=\frac{\partial \gamma\left(h_{i j} ; \boldsymbol{\theta}\right)}{\partial \theta_{k}}, k=$ $1,2, \ldots, q$ and $\boldsymbol{\theta}$ is a $k$ dimensional parameter. We now verify that Matérn covariance function satisfy Assumption 4 for fixed $\nu$. For Matérn covariance function with fixed $\nu$, we have

$$
\begin{aligned}
\frac{\partial \gamma(h)}{\partial \sigma^{2}} & =\frac{2^{1-\nu}}{\Gamma(\nu)}(h / r)^{\nu} K_{\nu}(h / r)(1-c) \\
\frac{\partial \gamma(h)}{\partial c} & =-\sigma^{2} \frac{2^{1-\nu}}{\Gamma(\nu)}(h / r)^{\nu} K_{\nu}(h / r) \\
\frac{\partial \gamma(h)}{\partial(1 / r)} & =\sigma^{2}(1-c) \frac{2^{1-\nu}}{\Gamma(\nu)} h(h / r)^{\nu}\left(2 \frac{\nu}{h / r} K_{\nu}(h / r)-K_{\nu-1}(h / r)\right)
\end{aligned}
$$

It is easy to show that for each $k$, exist a constant $\epsilon>0$ independent of $n, p$, for each $i$, there's $j$ such that $\gamma_{k}\left(h_{i j}\right)>c$. As a result, $\left\|\Sigma_{k}\right\|_{F}=\sum_{i, j=1}^{p} \gamma_{k}^{2}\left(h_{i j} ; \boldsymbol{\theta}\right) \geq$ $\sum_{i=1}^{p} \epsilon=p \epsilon$. Therefore $\left\|\Sigma_{k}\right\|_{F}^{-1}=O_{p}\left(p^{-1}\right)$.

\section{Remarks on Assumption 6:}

We can also verify that the Matérn covariance function with fixed $\nu$ satisfy Assumption 6 and Assumption 6. Similar to the procedure in the remarks on Assumption 3 , it is sufficient to verify for any $\boldsymbol{\theta} \in \Theta, \gamma_{k}(h ; \boldsymbol{\theta})$ and $\gamma_{k j}(h ; \boldsymbol{\theta})$ belong to the function space:

$$
\Im=\left\{f(x): \int_{0}^{\infty} f(x) x^{d-1} d x<\infty\right\}
$$

where $d \geq 1$ is the dimension of the domain. We have the first-order partial derivative of Matérn covariance function in (7.4). The second-order partial derivative of Matérn covariance function is as follows:

$$
\begin{aligned}
& \frac{\partial^{2} \gamma(h)}{\left(\partial^{2} \sigma^{2}\right)}=0 \\
& \frac{\partial^{2} \gamma(h)}{\partial \sigma^{2} \partial c}=-\frac{2^{1-\nu}}{\Gamma(\nu)}(h / r)^{\nu} K_{\nu}(h / r) \\
& \frac{\partial^{2} \gamma(h)}{\partial \sigma^{2} \partial(1 / r)}=(1-c) \frac{2^{1-\nu}}{\Gamma(\nu)} h(h / r)^{\nu}\left(2 \frac{\nu}{h / r} K_{\nu}(h / r)-K_{\nu-1}(h / r)\right) \\
& \frac{\partial^{2} \gamma(h)}{\partial c^{2}}=0 \\
& \frac{\partial^{2} \gamma(h)}{\partial c \partial(1 / r)}=-\sigma^{2} \frac{2^{1-\nu}}{\Gamma(\nu)} h(h / r)^{\nu}\left(2 \frac{\nu}{h / r} K_{\nu}(h / r)-K_{\nu-1}(h / r)\right) \\
& \frac{\partial^{2} \gamma(h)}{\partial^{2}(1 / r)}=\sigma^{2}(1-c) \frac{2^{1-\nu}}{\Gamma(\nu)}\left[(h / r)^{\nu-2} h^{2} K_{\nu}(h / r)(2 \nu-1) 2 \nu\right.
\end{aligned}
$$




$$
\left.-(4 \nu+1)(h / r)^{\nu-1} h^{2} K_{\nu+1}(h / r)-(h / r)^{\nu} h^{2} K_{\nu+2}(h / r)\right]
$$

Note that the covariance function and its first-order and second-order partial derivatives are linear combinations of a Bessel function of $h$ times a polynomial of $h$. Similar to proving $\int_{0}^{\infty} h^{d-1} \gamma(h) d h<\infty$ in (7.1), we have $\gamma_{k}(h ; \boldsymbol{\theta}) \in \Im$ and $\gamma_{k j}(h ; \boldsymbol{\theta}) \in \Im$. Hence Assumption 6 and Assumption 6 are satisfied. By similar procedure, we can verify that Matérn covariance function also satisfy Assumption 12 and Assumption 13.

\section{Appendix A}

\section{A.1. Proofs for classification using $M L E$}

Lemma 1. Let $\boldsymbol{\epsilon}$ be p-dimensional vectors and $\boldsymbol{\epsilon} \sim N(0, \Sigma)$, where $\Sigma$ is a $p \times p$ positive definite covariance matrix. For m-dimension vector $\boldsymbol{u}$ with $\|\boldsymbol{u}\|_{2}=$ $\sqrt{\boldsymbol{u}^{T} \boldsymbol{u}}=C$ and $p \times m$ matrix $\boldsymbol{X}_{i}$, we have:

$$
\left|\boldsymbol{\epsilon}^{T} \boldsymbol{X} u\right|=O_{p}\left(\sqrt{\operatorname{tr}\left(\boldsymbol{X}^{T} \Sigma \boldsymbol{X}\right)}\|\boldsymbol{u}\|_{2}\right)
$$

Proof. Since $E\left(\boldsymbol{\epsilon}^{T} \boldsymbol{X}\right)=0$,

$$
\begin{aligned}
E\left(\boldsymbol{\epsilon}^{T} \boldsymbol{X} \boldsymbol{u}\right)^{2} & \leq\left[E\left(\boldsymbol{\epsilon}^{T} \boldsymbol{X} \boldsymbol{X}^{T} \boldsymbol{\epsilon}\right)\right]^{1 / 2}\|\boldsymbol{u}\|_{2}=\left[E\left(\operatorname{tr}\left(\boldsymbol{\epsilon}^{T} \boldsymbol{X} \boldsymbol{X}^{T} \boldsymbol{\epsilon}\right)\right)\right]^{1 / 2}\|\boldsymbol{u}\|_{2} \\
& =\operatorname{tr}\left(\boldsymbol{X}^{T} \Sigma \boldsymbol{X}\right)\|\boldsymbol{u}\|_{2}
\end{aligned}
$$

By Chebyshev's inequality, for any $M$

$$
P\left(\frac{\boldsymbol{\epsilon}^{T} \boldsymbol{X} \boldsymbol{u}}{\sqrt{\|\boldsymbol{u}\|_{2}^{2} \operatorname{tr}\left(\boldsymbol{X}^{T} \Sigma \boldsymbol{X}\right)}}>M\right) \leq \frac{E\left(\boldsymbol{\epsilon}^{T} \boldsymbol{X} u\right)^{2}}{M^{2}\|\boldsymbol{u}\|_{2}^{2} \operatorname{tr}\left(\boldsymbol{X}^{T} \Sigma \boldsymbol{X}\right)}=\frac{1}{M^{2}}
$$

Thus for any $\epsilon>0$, exits $M$ large enough such that

$$
P\left(\frac{\left|\boldsymbol{\epsilon}^{T} \boldsymbol{X} \boldsymbol{u}\right|}{\sqrt{\|\boldsymbol{u}\|_{2}^{2} c(n) \operatorname{tr}\left(\boldsymbol{X}^{T} \Sigma \boldsymbol{X}\right)}}>M\right)<\epsilon
$$

This lead to $\left|\boldsymbol{\epsilon}^{T} \boldsymbol{X} u\right|=O_{p}\left(\sqrt{\operatorname{tr}\left(\boldsymbol{X}^{T} \Sigma \boldsymbol{X}\right)}\|\boldsymbol{u}\|_{2}\right)$.

Lemma 2. Let $\boldsymbol{\epsilon}_{i}(i=1,2, \ldots, n)$ be p-dimensional vectors and $\boldsymbol{\epsilon}_{i} \sim$ $N(0, c(n) \Sigma)$, where $c(n)$ is a function of $n$ and $\Sigma$ is a $p \times p$ positive definite covariance matrix with $\lambda(\Sigma)<\infty$. For a $p \times p$ matrix $A$, we have

$$
\sum_{i=1}^{n}\left[\boldsymbol{\epsilon}_{i}^{T} A \boldsymbol{\epsilon}_{i}-c(n) \operatorname{tr}(A \Sigma)\right]=O_{p}\left(c(n) \sqrt{n}\|A\|_{F}\right)
$$


Proof. Since $E\left(\boldsymbol{\epsilon}_{i}^{T} A \boldsymbol{\epsilon}_{i}\right)=\operatorname{tr}(c(n) A \Sigma)$, we have

$$
\begin{aligned}
& E\left(\sum_{i=1}^{n} \boldsymbol{\epsilon}_{i}^{T} A \boldsymbol{\epsilon}_{i}-c(n) \operatorname{tr}(A \Sigma)\right)^{2} \\
= & \sum_{i=1}^{n} E\left(\boldsymbol{\epsilon}_{i}^{T} A \boldsymbol{\epsilon}_{i}-c(n) \operatorname{tr}(A \Sigma)\right)^{2}=\sum_{i=1}^{n} E\left(\boldsymbol{\epsilon}_{i}^{T} A \boldsymbol{\epsilon}_{i}\right)^{2}-n c^{2}(n) \operatorname{tr}^{2}(A \Sigma)
\end{aligned}
$$

Let $B=c(n) \Sigma^{\frac{1}{2}} A \Sigma^{\frac{1}{2}}$, then exit orthogonal matrix $Q$ such that $B=Q^{T} \Lambda Q$ where $\Lambda=\operatorname{diag}\left(\lambda_{i}\right)$ and $\lambda_{i}$ are eigenvalues of $B$. Let $\tilde{\boldsymbol{\epsilon}}_{i}=\sqrt{c(n) Q \Sigma^{-\frac{1}{2}} \boldsymbol{\epsilon}_{i}}$, then $\tilde{\boldsymbol{\epsilon}}_{i} \sim N\left(0, I_{p \times p}\right)$ where $I_{p \times p}$ is identity matrix. Then

$$
\begin{aligned}
E\left(\boldsymbol{\epsilon}_{i}^{T} A \boldsymbol{\epsilon}_{i}\right)^{2} & =E\left(\tilde{\boldsymbol{\epsilon}}_{i}^{T} \Lambda \tilde{\boldsymbol{\epsilon}}_{i}\right)^{2}=E\left(\sum_{i=1}^{p} \lambda_{j} \tilde{\boldsymbol{\epsilon}}_{i j}^{2}\right)^{2} \\
& =E\left(\sum_{i=1}^{p} \lambda_{j}^{2} \tilde{\boldsymbol{\epsilon}}_{i j}^{4}+\sum_{j, k=1}^{p} \lambda_{j} \lambda_{k} \tilde{\boldsymbol{\epsilon}}_{i j}^{2} \tilde{\boldsymbol{\epsilon}}_{i j}^{2}\right)=2 \sum_{i=1}^{p} \lambda_{j}^{2}+\left(\sum_{i=1}^{p} \lambda_{j}\right)^{2} \\
& =2 \operatorname{tr}\left(B^{T} B\right)+\operatorname{tr}^{2}(B) \\
& =c(n)^{2}\left[2 \operatorname{tr}(\Sigma A \Sigma A)+\operatorname{tr}^{2}(A \Sigma)\right]
\end{aligned}
$$

Hence

$$
E\left(\sum_{i=1}^{n} \boldsymbol{\epsilon}_{i}^{T} A \boldsymbol{\epsilon}_{i}-c(n) \operatorname{tr}(A \Sigma)\right)^{2}=2 n c(n)^{2} \operatorname{tr}\left(\Sigma A^{T} \Sigma A\right) \leq 2 n c(n)^{2} \lambda_{\max }^{2}(\Sigma) \operatorname{tr}\left(A^{T} A\right)
$$

By Chebyshev's inequality, for any $M$ we have

$$
P\left(\frac{\sum_{i=1}^{n} \boldsymbol{\epsilon}_{i}^{T} A \boldsymbol{\epsilon}_{i}-c(n) \operatorname{tr}(A \Sigma)}{\sqrt{n c(n)^{2}\|A\|_{F}^{2}}}>M\right) \leq \frac{2 n c(n)^{2} \operatorname{tr}\left(\Sigma A^{T} \Sigma A\right)}{M^{2} n c(n)^{2}\|A\|_{F}^{2}} \leq \frac{2 \lambda_{\max }^{2}(\Sigma)}{M^{2}}
$$

Then for any $\epsilon>0$ exits $M$ large enough such that

$$
p\left(\frac{\sum_{i=1}^{n} \boldsymbol{\epsilon}_{i}^{T} A \boldsymbol{\epsilon}_{i}-c(n) \operatorname{tr}(A \Sigma)}{\left.\sqrt{n c(n)^{2}\|A\|_{F}^{2}}\right)}>M\right)<\epsilon
$$

which means $\sum_{i=1}^{n} \boldsymbol{\epsilon}_{i}^{T} A \boldsymbol{\epsilon}_{i}-c(n) \operatorname{tr}(A \Sigma)=O_{p}\left(c(n) \sqrt{n}\|A\|_{F}\right)$

Proof of Theorem 2.1. Take derivative with respect to $\boldsymbol{\mu}_{k}(k=1,2)$ with the function $L\left(\boldsymbol{\theta}, \boldsymbol{\mu}_{1}, \boldsymbol{\mu}_{2}\right)$ defined in (2.7). Considering $\Sigma^{-1}(\boldsymbol{\theta})$ is nonsingular, we have $\boldsymbol{\mu}_{k}=\hat{\boldsymbol{\mu}}_{k M L E}=\overline{\boldsymbol{Y}}_{k}$.

For $\hat{\boldsymbol{\theta}}_{M L E}$, we first consider the case of $p / n \rightarrow 0$. It is sufficient to prove that for any given $\epsilon>0$, there is a large constant $C$ such that for large $p$ and $n$, the 
smallest rate of convergence $\eta_{n, p}$ is $\sqrt{\frac{1}{n p}}$ such that we have

$$
P\left(\sup _{\|\boldsymbol{u}\|_{2}=C} L\left(\boldsymbol{\theta}_{0}+\boldsymbol{u} \eta_{n, p}, \hat{\boldsymbol{\mu}}_{1}, \hat{\boldsymbol{\mu}}_{2}\right)<L\left(\boldsymbol{\theta}_{0}, \hat{\boldsymbol{\mu}}_{1}, \hat{\boldsymbol{\mu}}_{2}\right)\right)>1-\epsilon
$$

where $\boldsymbol{u} \in \mathbb{R}^{q}$. This implies that there exists a local maximum for the function $L$ in the neighborhood of $\boldsymbol{\theta}_{0}$ with the radius at most proportional to $\eta_{n, p}$.

$$
\begin{aligned}
& L\left(\boldsymbol{\theta}_{0}+\boldsymbol{u} \eta_{n, p}, \hat{\boldsymbol{\mu}}_{1}, \hat{\boldsymbol{\mu}}_{2}\right)-L\left(\boldsymbol{\theta}_{0}, \hat{\boldsymbol{\mu}}_{1}, \hat{\boldsymbol{\mu}}_{2}\right) \\
& =\left(\frac{\partial L}{\partial \boldsymbol{\theta}}\left(\boldsymbol{\theta}_{0}\right)\right)^{T} \boldsymbol{u} \eta_{n, p}+\frac{1}{2} \boldsymbol{u}^{T}\left(\frac{\partial^{2} L}{\partial \boldsymbol{\theta} \partial \boldsymbol{\theta}^{T}}\left(\boldsymbol{\theta}^{*}\right)\right) \boldsymbol{u} \eta_{n, p}^{2} \\
& =-\frac{n}{2} \boldsymbol{u}^{T} T\left(\boldsymbol{\theta}_{0}\right) \boldsymbol{u} \eta_{n, p}^{2}+\left(\frac{\partial L}{\partial \boldsymbol{\theta}}\left(\boldsymbol{\theta}_{0}\right)\right)^{T} \boldsymbol{u} \eta_{n, p}+\frac{1}{2} \boldsymbol{u}^{T}\left(\frac{\partial^{2} L}{\partial \boldsymbol{\theta} \partial \boldsymbol{\theta}^{T}}\left(\boldsymbol{\theta}^{*}\right)+m n T\left(\boldsymbol{\theta}_{0}\right)\right) \boldsymbol{u} \eta_{n, p}^{2} \\
& =(I)+(I I)+(I I I)
\end{aligned}
$$

where $T\left(\boldsymbol{\theta}_{0}\right)$ is a $q \times q$ matrix with its $(i, j)$ th element $t_{i j}\left(\boldsymbol{\theta}_{0}\right)=\operatorname{tr}\left(\Sigma^{-1} \Sigma_{i} \Sigma^{-1} \Sigma_{j}\right)$.

From Assumption 4 and $5, t_{i j}=a_{i j}\left(t_{i i}\right)^{\frac{1}{2}}\left(t_{j j}\right)^{\frac{1}{2}} \geq a_{i j} \lambda_{\min }^{-2}(\Sigma)\left\|\Sigma_{i}\right\|_{F}\left\|\Sigma_{j}\right\|_{F}$. There exists a constant $\mathrm{M}$ such that

$$
\begin{aligned}
(I) & =-\frac{n}{2} \boldsymbol{u}^{T} T \boldsymbol{u} \eta_{n, p}^{2}=-\frac{n}{2} \sum_{i, j=1}^{q} t_{i j} u_{i} u_{j} \eta_{n, p}^{2} \leq-\frac{M n p}{2} \eta_{n, p}^{2}\|\boldsymbol{u}\|_{2}^{2} \\
(I I) & =-\frac{1}{2} \sum_{k=1}^{2} \sum_{i=1}^{n_{k}} \sum_{j=1}^{q}\left[\left(\boldsymbol{Y}_{k i}-\hat{\boldsymbol{\mu}}_{k}\right)^{T} \Sigma^{j}\left(\boldsymbol{Y}_{k i}-\hat{\boldsymbol{\mu}}_{k}\right)-\left(\frac{n_{k}-1}{n_{k}}\right) \operatorname{tr}\left(\Sigma \Sigma^{j}\right)\right] u_{\theta_{j}} \eta_{n, p} \\
& +\frac{1}{2} \sum_{j=1}^{q} \operatorname{tr}\left(\Sigma \Sigma^{j}\right) u_{\theta_{j}} \eta_{n, p} \\
& =(1)+(2)
\end{aligned}
$$

Because $\boldsymbol{Y}_{k i}-\hat{\boldsymbol{\mu}}_{k} \sim N\left(0, \frac{n_{k}-1}{n_{k}} \Sigma\right)$, by Lemma 2,

$$
\begin{aligned}
|(1)| & =O_{p}\left(\sum_{k=1}^{2} \frac{n_{k}-1}{\sqrt{n_{k}}}\left\|\Sigma^{j}\right\|_{F}\|\boldsymbol{u}\|_{2} \eta_{n, p}\right) \\
& =O_{p}\left(\sqrt{n}\left\|\Sigma^{j}\right\|_{F}\|\boldsymbol{u}\|_{2} \eta_{n, p}\right)=O_{p}\left(\sqrt{n p}\|\boldsymbol{u}\|_{2} \eta_{n, p}\right)
\end{aligned}
$$

The last equality is because from Assumption $6,\left\|\Sigma^{i}\right\|_{F}^{2} \leq\left\|\Sigma^{i}\right\|_{2}^{2}=p \lambda_{\max }^{2}\left(\Sigma^{i}\right)=$ $O(p)$.

Also by Assumption $6, \operatorname{tr}\left(\Sigma \Sigma^{j}\right)=\operatorname{tr}\left(\Sigma^{1 / 2} \Sigma^{j} \Sigma^{1 / 2}\right) \leq \lambda_{\max }\left(\Sigma^{j}\right) \operatorname{tr}(\Sigma)$. Then noticing that $\operatorname{tr}(\Sigma)=O(p)$, and $p / n \rightarrow 0$

$$
|(2)|=\left|\frac{1}{2} \sum_{j=1}^{q} \operatorname{tr}\left(\Sigma \Sigma^{j}\right) u_{j} \eta_{n, p}\right|=O_{p}\left(\operatorname{tr}(\Sigma) \eta_{n, p}\|\boldsymbol{u}\|_{2}\right)=O_{p}\left(p \eta_{n, p}\|\boldsymbol{u}\|_{2}\right)
$$


Thus

$$
(I I)=O_{p}\left((\sqrt{n p}+p) \eta_{n, p}\|\boldsymbol{u}\|_{2}\right)
$$

- If $p / n \rightarrow 0,(I I)=O_{p}(\sqrt{n p}) \eta_{n, p}$. By choosing sufficient large $C=\|\boldsymbol{u}\|_{2}$, the minimal rate of $\eta_{n, p}$ to have $(I I)$ be dominated by $(I)$ is $\eta_{n, p} \stackrel{=}{=}$ $O_{p}\left(\sqrt{\frac{1}{n p}}\right)$

- If $p / n \rightarrow C$ with $0<C \leq \infty,(I I)=O_{p}\left(p \eta_{n, p}\right)$. Then the minimal rate of $\eta_{n, p}$ to have $(I I)$ dominated by $(I)$ is $\eta_{n, p}=O_{p}\left(\sqrt{\frac{1}{n}}\right)$

Since

$$
\begin{aligned}
& \frac{\partial L}{\partial \theta_{j} \partial \theta_{l}}(\boldsymbol{\theta}) \\
= & \frac{n}{2}\left[\operatorname{tr}\left(\Sigma^{j}(\boldsymbol{\theta}) \Sigma(\boldsymbol{\theta})\right)+\operatorname{tr}\left(\Sigma^{j}(\boldsymbol{\theta}) \Sigma_{l}(\boldsymbol{\theta})\right)\right]-\frac{1}{2} \sum_{k=1}^{2} \sum_{i=1}^{n_{k}}\left(\boldsymbol{Y}_{k i}-\hat{\boldsymbol{\mu}}_{k}\right)^{T} \Sigma^{j l}(\boldsymbol{\theta})\left(\boldsymbol{Y}_{k i}-\hat{\boldsymbol{\mu}}_{k}\right)
\end{aligned}
$$

$(I I I)$ can be written as

$$
(I I I)
$$$$
=\sum_{j, l=1}^{q}\left[\sum_{k=1}^{2} \sum_{i=1}^{n_{k}}\left(\left(\boldsymbol{Y}_{k i}-\hat{\boldsymbol{\mu}}_{k}\right)^{T} \Sigma^{j l}\left(\boldsymbol{\theta}^{*}\right)\left(\boldsymbol{Y}_{k i}-\hat{\boldsymbol{\mu}}_{k}\right)-\left(\frac{n_{k}-1}{n_{k}}\right) \operatorname{tr}\left(\Sigma\left(\boldsymbol{\theta}_{0}\right) \Sigma^{j l}\left(\boldsymbol{\theta}^{*}\right)\right)\right)\right]
$$$$
\times-\frac{1}{2} u_{\theta_{j}} u_{\theta_{l}} \eta_{n, p}^{2}
$$$$
+\frac{n}{2 n_{1} n_{2}} \sum_{j, l=1}^{q} \operatorname{tr}\left(\Sigma^{j l}\left(\boldsymbol{\theta}^{*}\right) \Sigma\left(\boldsymbol{\theta}_{0}\right)\right) u_{\theta_{j}} u_{\theta_{l}} \eta_{n, p}^{2}
$$$$
+\frac{n}{2} \sum_{j, l=1}^{q}\left[\operatorname{tr}\left(\Sigma^{j k}\left(\boldsymbol{\theta}^{*}\right) \Sigma\left(\boldsymbol{\theta}^{*}\right)\right)-\operatorname{tr}\left(\Sigma^{j k}\left(\boldsymbol{\theta}^{*}\right) \Sigma\left(\boldsymbol{\theta}_{0}\right)\right)\right] u_{\theta_{j}} u_{\theta_{l}} \eta_{n, p}^{2}
$$$$
+\frac{n}{2} \sum_{j, l=1}^{q}\left[\operatorname{tr}\left(\Sigma^{j}\left(\boldsymbol{\theta}^{*}\right) \Sigma_{l}\left(\boldsymbol{\theta}^{*}\right)\right)-\operatorname{tr}\left(\Sigma^{j}\left(\boldsymbol{\theta}_{0}\right) \Sigma_{l}\left(\boldsymbol{\theta}_{0}\right)\right)\right] u_{\theta_{j}} u_{\theta_{l}} \eta_{n, p}^{2}
$$

$$
=(3)+(4)+(5)+(6)
$$

By Lemma 2 and Assumption 6,

$$
|(3)|=O_{p}\left(\sqrt{n}\left\|\Sigma^{j l}\left(\theta^{*}\right)\right\|_{F} \eta_{n, p}^{2}\right)=O_{p}\left(\sqrt{n p} \eta_{n, p}^{2}\right)
$$

For (4), by Assumption 6

$$
\operatorname{tr}\left(\Sigma^{j l}\left(\boldsymbol{\theta}^{*}\right) \Sigma\left(\boldsymbol{\theta}_{0}\right)\right)=O_{p}(p)
$$

thus $|(4)|=O_{p}\left(\frac{p}{n} \eta_{n, p}^{2}\right)$ It is easy to see $(3)$, (4) are dominated by $(I)$. For $(5)$,

$$
|(5)|=\left|\frac{1}{4} n \sum_{j, k=1}^{q} \operatorname{tr}\left(\Sigma^{k j}\left(\boldsymbol{\theta}^{*}\right)\left(\Sigma\left(\boldsymbol{\theta}_{0}\right)-\Sigma\left(\boldsymbol{\theta}^{*}\right)\right)\right) u_{\theta_{k}} u_{\theta_{j}} \eta_{n, p}^{2}\right| .
$$


Let $d_{i l}\left(\boldsymbol{\theta}^{*}\right)$ be the $i, l$ th entry of matrix $\Sigma^{k j}\left(\boldsymbol{\theta}^{*}\right), \gamma_{i l}(\boldsymbol{\theta})$ be the $i, l$ th entry of $\Sigma(\boldsymbol{\theta})$, then by Assumption 2 and Assumption 6

$$
\begin{aligned}
\operatorname{tr}\left(\Sigma^{k j}\left(\boldsymbol{\theta}^{*}\right)\left(\Sigma\left(\boldsymbol{\theta}_{0}\right)-\Sigma\left(\boldsymbol{\theta}^{*}\right)\right)\right) & =\sum_{i, l=1}^{p} d_{i l}\left(\boldsymbol{\theta}^{*}\right)\left(\gamma_{l i}\left(\boldsymbol{\theta}_{0}\right)-\gamma_{l i}\left(\boldsymbol{\theta}^{*}\right)\right) \\
& \leq \sum_{i, l=1}^{p}\left|d_{i l}\left(\boldsymbol{\theta}^{*}\right)\right|\left\|\frac{\partial \gamma_{i l}\left(\theta^{*}\right)}{\partial \boldsymbol{\theta}}\right\|_{2}\left\|\boldsymbol{\theta}_{0}-\boldsymbol{\theta}^{*}\right\|_{2} \\
& \leq \sum_{i, l=1}^{p} d_{i l}\left(\boldsymbol{\theta}_{2}^{*}\right) M \eta_{n, p} \\
& \leq M \eta_{n, p} p\left\|\Sigma^{k j}\left(\boldsymbol{\theta}^{*}\right)\right\|_{F} \\
& =O_{p}\left(\sqrt{p^{3}} \eta_{n, p}\right)
\end{aligned}
$$

Hence

$$
\left.|(5)|=O_{P}\left(n \sqrt{p^{3}} \eta^{3}\right)=O_{p}\left(\left(p^{3 / 2} n \eta_{n, p}\right) \eta_{n, p}^{2}\right)\right)
$$

- If $p / n \rightarrow 0$ and $\eta_{n, p}=O_{p}\left(\frac{1}{\sqrt{n p}}\right),(5)$ is dominated by $(I)$.

- If $p / n \rightarrow C$ with $0<C \leq \infty$, eta $a_{n, p}=O_{p}\left(\frac{1}{n}\right)$ and $\sqrt{p} / n \rightarrow 0$, (5) is dominated by $(I)$.

For (6), let $t_{i j}\left(\theta^{*}\right)=\operatorname{tr}\left(\Sigma^{-1}\left(\boldsymbol{\theta}^{*}\right) \Sigma_{i}\left(\boldsymbol{\theta}^{*}\right) \Sigma^{-1}\left(\boldsymbol{\theta}^{*}\right) \Sigma_{j}\left(\boldsymbol{\theta}^{*}\right)\right)$, by Assumption 6 ,

$$
\begin{aligned}
|(6)| & \leq n \sum_{k, j=1}^{q}\left\|\frac{\partial t_{i j}\left(\boldsymbol{\theta}^{*}\right)}{\partial \boldsymbol{\theta}}\right\|_{2}\left\|\boldsymbol{\theta}^{*}-\boldsymbol{\theta}_{0}\right\|_{2} u_{\theta_{k}} u_{\theta_{j}} \eta_{n, p}^{2} \\
& =O_{P}\left(n p \eta_{n, p}^{3}\right)
\end{aligned}
$$

While $\eta_{n, p}=O_{p}\left(\frac{1}{\sqrt{n p}}\right)$ or $\eta_{n, p}=O_{p}\left(\frac{1}{n}\right)$, (6) is also dominated by $(I)$. Hence $(I I I)$ is dominated by $(I)$. This completes the proof.

Proof of Theorem 2.2. We start with $W_{1}\left(\hat{\delta}_{M L E}\right)=1-\Phi\left(\Psi_{1}\right)$, where $W_{1}\left(\hat{\delta}_{M L E}\right)$ is the conditional misclassification rate defined in (1.2) and $\Psi_{1}$ is defined in (1.3). The idea is to prove $\liminf _{n, p \rightarrow 0} \Psi_{1} \rightarrow \frac{\sqrt{C_{0}}}{2}$.

From Theorem 2.1, we have $\|\hat{\boldsymbol{\theta}}-\boldsymbol{\theta}\|_{2}=O_{p}\left(\frac{1}{\sqrt{n p}}\right)$. Recall that $\Sigma=\Sigma(\boldsymbol{\theta})=$ $\left[\gamma\left(h_{i j} ; \boldsymbol{\theta}\right)\right]_{i, j=1}^{p}$ and $\hat{\Sigma}=\Sigma(\hat{\boldsymbol{\theta}})=\left[\gamma\left(h_{i j} ; \hat{\boldsymbol{\theta}}\right)\right]_{i, j=1}^{p}$.

By Assumption 2, we have:

$$
\max _{i, j}\left|\gamma\left(h_{i j} ; \boldsymbol{\theta}\right)-\gamma\left(h_{i j} ; \hat{\boldsymbol{\theta}}\right)\right| \leq M\|\boldsymbol{\theta}-\hat{\boldsymbol{\theta}}\|_{2} .
$$

Thus there exist $\epsilon>0$ and matrix $E=\left[e_{i j}\right]_{i, j=1}^{p}$ such that

$$
\hat{\Sigma}=\Sigma+\epsilon E,
$$


where $\epsilon=O_{p}\left(\frac{1}{\sqrt{n p}}\right)$ and $E$ is a $p \times p$ matrix with absolute values of all entries less than 1 , i.e. $\left|e_{i j}\right| \leq 1$ for any $i, j=1,2, \ldots, p$. As a result, for large $p$ and $n$, the inverse of $\Sigma$ can be written as:

$$
\hat{\Sigma}^{-1}=\Sigma^{-1}-\epsilon \Sigma^{-1} E \Sigma^{-1}+O\left(\epsilon^{2}\right) E_{2}
$$

where $\mathbb{E}_{2}$ is a $p \times p$ matrix with all entries less than 1 , see Meyer [32].

Now we consider the denominator of (1.3). We first claim the denominator can be written as:

$$
\hat{\boldsymbol{\Delta}}^{T}\left(\hat{\Sigma}^{-1} \Sigma \hat{\Sigma}^{-1}\right) \hat{\boldsymbol{\Delta}}=\hat{\boldsymbol{\Delta}}^{T} \Sigma^{-1} \hat{\boldsymbol{\Delta}}\left(1+o_{p}(1)\right) .
$$

Because by (A.21), we have

$$
\begin{aligned}
\hat{\Sigma}^{-1} \Sigma \hat{\Sigma}^{-1} & =\left(\Sigma^{-1}-\epsilon \Sigma^{-1} E \Sigma^{-1}+O\left(\epsilon^{2}\right) E_{2}\right) \Sigma\left(\Sigma^{-1}-\epsilon \Sigma^{-1} E \Sigma^{-1}+O\left(\epsilon^{2}\right) E_{2}\right) \\
& =\Sigma^{-1}-2 \epsilon A+\epsilon^{2} A \Sigma A+O\left(\epsilon^{2}\right) E_{2}+O\left(\epsilon^{3}\right) E E_{2} \Sigma^{-1}+O\left(\epsilon^{4}\right) E_{2} E_{2}
\end{aligned}
$$

where $A=\Sigma^{-1} E \Sigma^{-1}$

Also, noticing that $\epsilon=O\left(\frac{1}{\sqrt{n p}}\right), k_{1} \leq \lambda_{\min }(\Sigma) \leq \lambda_{\max }(\Sigma) \leq k_{2}$ and $\lambda_{\max }(E) \leq \operatorname{tr}(E) \leq p$, we have:

$$
\frac{\hat{\boldsymbol{\Delta}}^{T}(\epsilon A) \hat{\boldsymbol{\Delta}}}{\hat{\boldsymbol{\Delta}}^{T} \Sigma^{-1} \hat{\boldsymbol{\Delta}}}=\frac{y^{T} \epsilon E y}{y^{T} \Sigma y} \leq \frac{\epsilon \lambda_{\max }(E)}{\lambda_{\min }(\Sigma)} \leq \epsilon p / \lambda_{\min }(\Sigma)=O\left(\sqrt{\frac{p}{n}}\right)
$$

where $y^{T}=\hat{\boldsymbol{\Delta}}^{T} \Sigma^{-1}$. Similarly, we have:

$$
\begin{aligned}
\frac{\hat{\boldsymbol{\Delta}}^{T}\left(\epsilon^{2} A \Sigma A\right) \hat{\boldsymbol{\Delta}}}{\hat{\boldsymbol{\Delta}}^{T} \Sigma^{-1} \hat{\boldsymbol{\Delta}}} & \leq \epsilon^{2} \frac{\lambda_{\max }^{2}(E)}{\lambda_{\min }^{2}(\Sigma)} \leq \epsilon^{2} p^{2} / \lambda_{\min }^{2}(\Sigma)=O\left(\frac{p}{n}\right) \\
\frac{\hat{\boldsymbol{\Delta}}^{T}\left(O\left(\epsilon^{2}\right) E_{2}\right) \hat{\boldsymbol{\Delta}}}{\hat{\boldsymbol{\Delta}}^{T} \Sigma^{-1} \hat{\boldsymbol{\Delta}}} & \leq O\left(\epsilon^{2}\right) \lambda_{\max }\left(E_{2}\right) \lambda_{\min }(\Sigma) \leq O\left(\epsilon^{2}\right) p \lambda_{\max }(\Sigma)=O\left(\frac{1}{n}\right) \\
\frac{\hat{\boldsymbol{\Delta}}^{T}\left(O\left(\epsilon^{3}\right) E E_{2} \Sigma^{-1}\right) \hat{\boldsymbol{\Delta}}}{\hat{\boldsymbol{\Delta}}^{T} \Sigma^{-1} \hat{\boldsymbol{\Delta}}} & \leq O\left(\epsilon^{3}\right) \frac{\lambda_{\max }\left(E_{2}\right) \lambda_{\max }(E) \lambda_{\max }\left(\Sigma^{-1}\right)}{\lambda_{\min }(\Sigma)} \\
& \leq O\left(\epsilon^{3}\right) p^{2} \frac{\lambda_{\max }(\Sigma)}{\lambda_{\min }(\Sigma)}=O\left(\sqrt{\frac{p}{n}} \frac{1}{n}\right) \\
\frac{\hat{\boldsymbol{\Delta}}^{T}\left(O\left(\epsilon^{4}\right) E_{2} E_{2}\right) \hat{\boldsymbol{\Delta}}}{\hat{\boldsymbol{\Delta}}^{T} \Sigma^{-1} \hat{\boldsymbol{\Delta}}} & \leq O\left(\epsilon^{4}\right) p^{2} \lambda_{\max }(\Sigma)=O_{p}\left(\frac{1}{n^{2}}\right)
\end{aligned}
$$

Since $\frac{p}{n} \rightarrow 0$ as $n \rightarrow \infty$ and $p \rightarrow \infty$, (A.22) is derived by combining (A.24)(A.28).

Now we investigate $\hat{\boldsymbol{\Delta}}^{T} \Sigma^{-1} \hat{\boldsymbol{\Delta}}$ and claim that:

$$
\hat{\boldsymbol{\Delta}}^{T} \Sigma^{-1} \hat{\boldsymbol{\Delta}}=\Delta^{T} \Sigma^{-1} \Delta\left(1+o_{p}(1)\right)+\frac{n p}{n_{1} n_{2}}\left(1+o_{p}(1)\right)
$$


Recall $\hat{\boldsymbol{\mu}}_{1}=\overline{\boldsymbol{Y}}_{1}=\frac{1}{n_{1}} \sum_{i=1}^{n_{1}} \boldsymbol{Y}_{1 i}$, which is normally distributed as $\mathcal{N}\left(\boldsymbol{\mu}_{1}, \frac{1}{n_{1}} \Sigma\right)$. Also, $\hat{\boldsymbol{\mu}}_{2}=\overline{\boldsymbol{Y}}_{2}=\frac{1}{n_{2}} \sum_{i=1}^{n_{2}} \boldsymbol{Y}_{2 i}$, which is normally distributed as $N\left(\boldsymbol{\mu}_{2}, \frac{1}{n_{2}} \Sigma\right)$. Let $\hat{\boldsymbol{\mu}}_{1}=\boldsymbol{\mu}_{1}+\hat{\boldsymbol{\epsilon}}_{1}$ and $\hat{\boldsymbol{\mu}}_{2}=\boldsymbol{\mu}_{2}+\hat{\boldsymbol{\epsilon}}_{2}$ where $\hat{\boldsymbol{\epsilon}}_{1} \sim \mathcal{N}\left(0, \frac{1}{n_{1}} \Sigma\right)$ and $\hat{\boldsymbol{\epsilon}}_{2} \sim N\left(0, \frac{1}{n_{2}} \Sigma\right)$. Then we have:

$$
\hat{\boldsymbol{\Delta}}^{T} \Sigma^{-1} \hat{\boldsymbol{\Delta}}=\boldsymbol{\Delta}^{T} \Sigma^{-1} \boldsymbol{\Delta}+2 \Delta^{T} \Sigma^{-1}\left(\hat{\boldsymbol{\epsilon}}_{1}-\hat{\boldsymbol{\epsilon}}_{2}\right)+\left(\hat{\boldsymbol{\epsilon}}_{1}-\hat{\boldsymbol{\epsilon}}_{2}\right)^{T} \Sigma^{-1}\left(\hat{\boldsymbol{\epsilon}}_{1}-\hat{\boldsymbol{\epsilon}}_{2}\right)
$$

Noticing $\hat{\boldsymbol{\epsilon}}_{1}-\hat{\boldsymbol{\epsilon}}_{2} \sim N\left(0, \frac{n}{n_{1} n_{2}} \Sigma\right)$, by Chebyshev's inequality, for any $\epsilon_{0}>0$

$$
\begin{aligned}
P\left(\frac{\boldsymbol{\Delta}^{T} \Sigma^{-1}\left(\hat{\boldsymbol{\epsilon}}_{1}-\hat{\boldsymbol{\epsilon}}_{2}\right)}{\boldsymbol{\Delta}^{T} \Sigma^{-1} \boldsymbol{\Delta}}>\epsilon_{0}\right) & \leq \frac{E\left(\boldsymbol{\Delta}^{T} \Sigma^{-1}\left(\hat{\epsilon}_{1}-\hat{\epsilon}_{2}\right)\right)^{2}}{\left(\epsilon_{0} \boldsymbol{\Delta}^{T} \Sigma^{-1} \boldsymbol{\Delta}\right)^{2}} \\
& =\frac{n}{n_{1} n_{2} \boldsymbol{\Delta}^{T} \Sigma^{-1} \boldsymbol{\Delta}} \\
& \leq \frac{n}{n_{1} n_{2} C_{p}}=\frac{1}{\pi(1-\pi) n C_{p}} \rightarrow 0
\end{aligned}
$$

It goes to 0 because $n C_{p} \rightarrow \infty$. Then

$$
\boldsymbol{\Delta}^{T} \Sigma^{-1}\left(\hat{\boldsymbol{\epsilon}}_{1}-\hat{\boldsymbol{\epsilon}}_{2}\right)=o_{p}\left(\boldsymbol{\Delta}^{T} \Sigma^{-1} \boldsymbol{\Delta}\right)
$$

Then we consider the third term in (A.29). Let $\tilde{\boldsymbol{\epsilon}}=\sqrt{\frac{n_{1} n_{2}}{n}} \sum^{\frac{1}{2}}\left(\hat{\boldsymbol{\epsilon}}_{1}-\hat{\boldsymbol{\epsilon}}_{2}\right)$. Then $\tilde{\boldsymbol{\epsilon}} \sim \mathcal{N}\left(0, I_{p \times p}\right)$. Now for any $\varepsilon_{0}>0$

$$
\begin{aligned}
P\left(\left|\frac{\left(\hat{\boldsymbol{\epsilon}}_{1}-\hat{\boldsymbol{\epsilon}}_{2}\right)^{T} \Sigma^{-1}\left(\hat{\boldsymbol{\epsilon}}_{1}-\hat{\boldsymbol{\epsilon}}_{2}\right)-n p / n_{1} n_{2}}{n p / n_{1} n_{2}}\right|>\epsilon_{0}\right) & =P\left(\left|\frac{\tilde{\boldsymbol{\epsilon}}^{T} \tilde{\boldsymbol{\epsilon}}-p}{p}\right|>\epsilon_{0}\right) \\
& \leq \frac{E\left(\tilde{\boldsymbol{\epsilon}}^{T} \tilde{\boldsymbol{\epsilon}}\right)^{2}}{\epsilon_{0}^{2} p^{2}} \\
& =\frac{2}{p} \frac{1}{\epsilon^{2}} \rightarrow 0
\end{aligned}
$$

as $p \rightarrow \infty$. Then

$$
\left(\hat{\boldsymbol{\epsilon}}_{1}-\hat{\boldsymbol{\epsilon}}_{2}\right)^{T} \Sigma^{-1}\left(\hat{\boldsymbol{\epsilon}}_{1}-\hat{\boldsymbol{\epsilon}}_{2}\right)=\frac{n p}{n_{1} n_{2}}\left(1+o_{p}(1)\right)
$$

Then (A.29) followed. Now (A.22) and (A.29) yield:

$$
\hat{\boldsymbol{\Delta}}^{T}\left(\hat{\Sigma}^{-1} \Sigma \hat{\Sigma}^{-1}\right) \hat{\boldsymbol{\Delta}}=\boldsymbol{\Delta}^{T} \Sigma \boldsymbol{\Delta}\left(1+o_{p}(1)\right)+\frac{n p}{n_{1} n_{2}}\left(1+o_{p}(1)\right)
$$

Now we consider the nominator of (1.3).

$$
\begin{aligned}
\left(\boldsymbol{\mu}_{1}-\hat{\boldsymbol{\mu}}\right)^{T} \hat{\Sigma} \hat{\boldsymbol{\Delta}} & =\frac{1}{2}\left(\boldsymbol{\Delta}^{T} \hat{\Sigma}^{-1} \boldsymbol{\Delta}+\hat{\boldsymbol{\epsilon}}_{2}^{T} \hat{\Sigma}^{-1} \hat{\boldsymbol{\epsilon}}_{2}-\hat{\boldsymbol{\epsilon}}_{1}^{T} \hat{\Sigma}^{-1} \hat{\boldsymbol{\epsilon}}_{1}-2 \boldsymbol{\Delta}^{T} \hat{\Sigma}^{-1} \hat{\boldsymbol{\epsilon}}_{2}\right) \\
& =\frac{1}{2}((1)+(2)-(3)-(4))
\end{aligned}
$$




$$
(1)=\boldsymbol{\Delta}^{T}\left(\Sigma^{-1}-\epsilon E+O\left(\epsilon^{2}\right) E_{2}\right) \boldsymbol{\Delta}
$$

By the assumption that $k_{1} \leq \lambda_{\min }(\Sigma) \leq \lambda_{\max } \leq k_{2}, \lambda_{\max }(E) \leq p$ and $\lambda_{\max }\left(E_{2}\right) \leq p$, we have

$$
\frac{\boldsymbol{\Delta}^{T}(\epsilon E) \boldsymbol{\Delta}}{\boldsymbol{\Delta}^{T \Sigma^{-1} \boldsymbol{\Delta}}} \rightarrow 0
$$

and

$$
\frac{\boldsymbol{\Delta}^{T}\left(O\left(\epsilon^{2}\right) E_{2}\right) \boldsymbol{\Delta}}{\boldsymbol{\Delta}^{T} \Sigma^{-1} \boldsymbol{\Delta}} \rightarrow 0
$$

thus

$$
(1)=\boldsymbol{\Delta}^{T} \Sigma^{-1} \boldsymbol{\Delta}\left(1+o_{p}(1)\right)
$$

By the same argument, we have $(2)=\hat{\boldsymbol{\epsilon}}_{2}^{T} \Sigma^{-1} \hat{\boldsymbol{\epsilon}}_{2}\left(1+o_{p}(1)\right)$ and $(3)=$ $\hat{\boldsymbol{\epsilon}}_{1}^{T} \Sigma^{-1} \hat{\boldsymbol{\epsilon}}_{1}\left(1+o_{p}(1)\right)$. Since $\hat{\boldsymbol{\epsilon}}_{1} \sim N\left(0, \frac{1}{n_{1}} \Sigma\right)$ and $\hat{\boldsymbol{\epsilon}}_{1} \sim N\left(0, \frac{1}{n_{1}} \Sigma\right)$, similar to the proof of (A.31), we have:

$$
(2)=\frac{p}{n_{2}}\left(1+o_{p}(1)\right) \text { and }(3)=\frac{p}{n_{1}}\left(1+o_{p}(1)\right)
$$

Now we consider term (4) in (A.33).

$$
\begin{aligned}
(4) & =\boldsymbol{\Delta}^{T}\left(\Sigma^{-1}-\epsilon \Sigma^{-1} E \Sigma^{-1}+O\left(\epsilon^{2}\right) E_{2}\right) \hat{\boldsymbol{\epsilon}}_{2} \\
& =\boldsymbol{\Delta}^{T} \Sigma^{-1} \hat{\boldsymbol{\epsilon}}_{2}+\epsilon \boldsymbol{\Delta}^{T} \Sigma^{-1} E \Sigma^{-1} \hat{\boldsymbol{\epsilon}}_{2}+O\left(\epsilon^{2}\right) \boldsymbol{\Delta}^{T} E_{2} \hat{\boldsymbol{\epsilon}}_{2}
\end{aligned}
$$

Similar to the proof of (A.30), all the three terms in (4) are small order of $\boldsymbol{\Delta}^{T} \Sigma^{-1} \boldsymbol{\Delta}$. Thus we have

$$
(4)=o_{p}\left(\boldsymbol{\Delta}^{T} \Sigma^{-1} \boldsymbol{\Delta}\right)
$$

Now the nominator can be written as:

$$
\left(\boldsymbol{\mu}_{1}-\hat{\boldsymbol{\mu}}\right)^{T} \hat{\Sigma}^{-1} \hat{\boldsymbol{\Delta}}=\frac{1}{2}\left(\boldsymbol{\Delta}^{T} \Sigma^{-1} \boldsymbol{\Delta}\left(1+o_{p}(1)\right)+\frac{p}{n_{1} n_{2}}\left(n_{1}-n_{2}\right)\left(1+o_{p}(1)\right)\right)
$$

(A.32) and (A.37) yield

$$
W_{1}\left(\hat{\delta}_{M L E}\right)=1-\Phi\left(\frac{\boldsymbol{\Delta}^{T} \Sigma^{-1} \boldsymbol{\Delta}\left(1+o_{p}(1)\right)+\frac{p}{n_{1} n_{2}}\left(n_{1}-n_{2}\right)\left(1+o_{p}(1)\right)}{2 \sqrt{\boldsymbol{\Delta}^{T} \Sigma^{-1} \boldsymbol{\Delta}\left(1+o_{p}(1)\right)+\frac{n p}{n_{1} n_{2}}\left(1+o_{p}(1)\right)}}\right)
$$

By the same argument, we have:

$$
W_{2}\left(\hat{\delta}_{M L E}\right)=\Phi\left(\frac{-\boldsymbol{\Delta}^{T} \Sigma^{-1} \boldsymbol{\Delta}\left(1+o_{p}(1)\right)+\frac{p}{n_{1} n_{2}}\left(n_{1}-n_{2}\right)\left(1+o_{p}(1)\right)}{2 \sqrt{\boldsymbol{\Delta}^{T} \Sigma^{-1} \boldsymbol{\Delta}\left(1+o_{p}(1)\right)+\frac{n p}{n_{1} n_{2}}\left(1+o_{p}(1)\right)}}\right)
$$


Since $\frac{p}{n} \rightarrow 0$ and $C_{p}=\Delta^{T} \Sigma^{-1} \Delta \rightarrow C_{0}$ with $0 \leq C_{0} \leq \infty$, we have

$$
\begin{aligned}
W\left(\hat{\delta}_{M L E}\right)= & \frac{1}{2}\left(1-\Phi\left(\frac{C_{p}\left(1+o_{p}(1)\right)+\frac{p}{n_{1} n_{2}}\left(n_{1}-n_{2}\right)\left(1+o_{p}(1)\right)}{2 \sqrt{C_{p}\left(1+o_{p}(1)\right)+\frac{n p}{n_{1} n_{2}}\left(1+o_{p}(1)\right)}}\right)\right. \\
& \left.+\Phi\left(\frac{-C_{p}\left(1+o_{p}(1)\right)+\frac{p}{n_{1} n_{2}}\left(n_{1}-n_{2}\right)\left(1+o_{p}(1)\right)}{2 \sqrt{C_{p}\left(1+o_{p}(1)\right)+\frac{n p}{n_{1} n_{2}}\left(1+o_{p}(1)\right)}}\right)\right) \\
& \rightarrow 1-\Phi\left(\frac{\sqrt{C_{0}}}{2}\right)
\end{aligned}
$$

as $p \rightarrow \infty$ and $n \rightarrow \infty$. If $C_{p} \rightarrow C_{0}<\infty, 1-\Phi\left(\frac{\sqrt{C_{0}}}{2}\right)>0$. Thus $\hat{\delta}_{M L E}$ is asymptotically optimal. Now we check the asymptotically optimal when $C_{p} \rightarrow \infty$. From the inequality

$$
\frac{x}{1+x^{2}} e^{-\frac{x^{2}}{2}} \leq \Phi(-x) \leq \frac{1}{x} e^{-\frac{x^{2}}{2}}, x>0
$$

we have

$$
\frac{x y}{1+x^{2}} e^{-\frac{x^{2}-y^{2}}{2}} \leq \frac{W\left(\hat{\delta}_{M L E}\right)}{\Phi\left(-\frac{\sqrt{C_{p}}}{2}\right)} \leq \frac{1+y^{2}}{x y} e^{-\frac{x^{2}-y^{2}}{2}}
$$

where $x=\frac{C_{p}\left(1+o_{p}(1)\right) \pm \frac{p}{n_{1} n_{2}}\left(n_{1}-n_{2}\right)\left(1+o_{p}(1)\right)}{2 \sqrt{C_{p}\left(1+o_{p}(1)\right)+\frac{n p}{n_{1} n_{2}}\left(1+o_{p}(1)\right)}}$ and $y=\frac{\sqrt{C_{p}}}{2}$. It is easy to check that $\frac{x y}{1+x^{2}} \rightarrow 1$ and $\frac{1+y^{2}}{x y} \rightarrow 1$ as $C_{p} \rightarrow \infty$. Also $x^{2}-y^{2} \rightarrow 0$ if $C_{p}(p / n) \rightarrow 0$. This completes the proof.

Proof of Theorem 2.3. The misclassification rate of $\delta_{\hat{\mu}}$ is:

$$
W\left(\delta_{\hat{\boldsymbol{\mu}}}\right)=\frac{1}{2}\left(W_{1}\left(\delta_{\hat{\boldsymbol{\mu}}}\right)+W_{2}\left(\delta_{\hat{\boldsymbol{\mu}}}\right)\right)
$$

where

$$
W_{1}\left(\delta_{\hat{\boldsymbol{\mu}}}\right)=1-\Phi\left(\Psi_{1}\right) \text { and } W_{2}\left(\delta_{\hat{\boldsymbol{\mu}}}\right)=\Phi\left(\Psi_{2}\right)
$$

where $\Psi_{1}$ and $\Psi_{2}$ is defined by (1.3) and (1.5) with $\hat{\Sigma}$ replaced by $\Sigma$. We start with

$$
\Psi_{1}=\frac{\left(\boldsymbol{\mu}_{1}-\hat{\boldsymbol{\mu}}\right)^{T} \Sigma^{-1}\left(\hat{\boldsymbol{\mu}}_{1}-\hat{\boldsymbol{\mu}}_{2}\right)}{\sqrt{\left(\hat{\boldsymbol{\mu}}_{1}-\hat{\boldsymbol{\mu}}_{2}\right)^{T} \Sigma^{-1}\left(\hat{\boldsymbol{\mu}}_{1}-\hat{\boldsymbol{\mu}}_{2}\right)}}
$$

The denominator is (A.29) and it can be represented as:

$$
\hat{\boldsymbol{\Delta}} \Sigma^{-1} \hat{\boldsymbol{\Delta}}=\boldsymbol{\Delta}^{T} \Sigma \boldsymbol{\Delta}\left(1+o_{p}(1)\right)+\frac{n p}{n_{1} n_{2}}\left(1+o_{p}(1)\right)
$$

The nominator is:

$$
\left(\boldsymbol{\mu}_{1}-\hat{\boldsymbol{\mu}}\right)^{T} \Sigma^{-1} \hat{\boldsymbol{\Delta}}=\frac{1}{2}\left(\boldsymbol{\Delta}^{T} \Sigma^{-1} \boldsymbol{\Delta}+\hat{\boldsymbol{\epsilon}}_{1}^{T} \Sigma^{-1} \hat{\boldsymbol{\epsilon}}_{1}-\hat{\boldsymbol{\epsilon}}_{2}^{T} \Sigma^{-1} \hat{\boldsymbol{\epsilon}}_{2}-2 \boldsymbol{\Delta}^{T} \Sigma^{-1} \hat{\boldsymbol{\epsilon}}_{2}\right)
$$


By the similar procedure in the proof of (A.37), it can be represented by

$$
\left(\boldsymbol{\mu}_{1}-\hat{\boldsymbol{\mu}}\right)^{T} \Sigma^{-1} \hat{\boldsymbol{\Delta}}=\frac{1}{2}\left(\boldsymbol{\Delta}^{T} \Sigma^{-1} \boldsymbol{\Delta}\left(1+o_{p}(1)\right)+\frac{p}{n_{1} n_{2}}\left(n_{1}-n_{2}\right)\left(1+o_{p}(1)\right)\right)
$$

Thus we have:

$$
W_{1}\left(\hat{\delta}_{\hat{\boldsymbol{\mu}}}\right)=1-\Phi\left(\frac{C_{p}\left(1+o_{p}(1)\right)+\frac{p}{n_{1} n_{2}}\left(n_{1}-n_{2}\right)\left(1+o_{p}(1)\right)}{2 \sqrt{C_{p}\left(1+o_{p}(1)\right)+\frac{n p}{n_{1} n_{2}}\left(1+o_{p}(1)\right)}}\right)
$$

Similarly, we have

$$
W_{2}\left(\hat{\delta}_{\hat{\boldsymbol{\mu}}}\right)=\Phi\left(\frac{-C_{p}\left(1+o_{p}(1)\right)+\frac{p}{n_{1} n_{2}}\left(n_{1}-n_{2}\right)\left(1+o_{p}(1)\right)}{2 \sqrt{C_{p}\left(1+o_{p}(1)\right)+\frac{n p}{n_{1} n_{2}}\left(1+o_{p}(1)\right)}}\right)
$$

(i) If $\frac{C_{p}}{p / n} \rightarrow \infty$. Then

$$
\begin{aligned}
& \frac{ \pm C_{p}\left(1+o_{p}(1)\right)+\frac{p}{n_{1} n_{2}}\left(n_{1}-n_{2}\right)\left(1+o_{p}(1)\right)}{2 \sqrt{C_{p}\left(1+o_{p}(1)\right)+\frac{n p}{n_{1} n_{2}}\left(1+o_{p}(1)\right)}} \\
= & \frac{ \pm C_{p}\left(1 \pm \frac{p}{n_{1} n_{2} C_{p}}\left(n_{1}-n_{2}\right)\left(1+o_{p}(1)\right)\right)}{2 \sqrt{C_{p}\left(1+\frac{n p}{n_{1} n_{2} C_{p}}\left(1+o_{p}(1)\right)\right.}} \\
= & \frac{ \pm \sqrt{C_{p}}\left(1 \pm \frac{p}{n_{1} n_{2} C_{p}}\left(n_{1}-n_{2}\right)\left(1+o_{p}(1)\right)\right)}{2 \sqrt{\left(1+\frac{n p}{n_{1} n_{2} C_{p}}\left(1+o_{p}(1)\right)\right.}} \\
\rightarrow & \frac{ \pm \sqrt{C_{0}}}{2}
\end{aligned}
$$

which yields $W\left(\hat{\delta}_{\hat{\boldsymbol{\mu}}}\right) \rightarrow 0$ since $\frac{p}{n} \rightarrow C$ with $0<C<\infty$ and $C_{p} \rightarrow C_{0}=\infty$. Now we show that $\frac{W\left(\hat{\delta}_{\hat{\mu}}\right)}{W O P T} \rightarrow \infty$ in probability.

Noticing the fact that

$$
\frac{x}{1+x^{2}} e^{-\frac{x^{2}}{2}} \leq \Phi(-x) \leq \frac{1}{x} e^{-\frac{x^{2}}{2}}, x>0
$$

we have

$$
\frac{W_{O P T}}{\Phi\left(-\frac{x}{2}\right)}=\frac{\Phi\left(-\frac{\sqrt{C_{p}}}{2}\right)}{\Phi\left(-\frac{x}{2}\right)} \leq \frac{4+x^{2}}{x \sqrt{C_{p}}} e^{-\frac{1}{8}\left(C_{p}-x^{2}\right)}
$$

where $x=\frac{C_{p}\left(1+o_{p}(1) \pm \frac{p\left(n_{1}-n_{2}\right)}{n_{1} n_{2}}\left(1+o_{p}(1)\right)\right)}{\sqrt{C_{p}\left(1+o_{p}(1)\right)+\frac{n_{p}}{n_{1} n_{2}}\left(1+o_{p}(1)\right)}}$

$$
\frac{4+x^{2}}{x \sqrt{C_{p}}}=\frac{4}{x \sqrt{C_{p}}}+\frac{x}{\sqrt{C_{p}}} \rightarrow \text { a constant }
$$


because

$$
\frac{1}{x \sqrt{C_{p}}} \rightarrow 0
$$

and

$$
\frac{x}{\sqrt{C_{p}}}= \begin{cases}\rightarrow 1 & \text { if } c=\infty \\ \rightarrow a_{0} & \text { if } c<\infty\end{cases}
$$

where $a_{0}=\frac{\sqrt{c}+1 / \sqrt{c}}{\sqrt{c+1 /(\pi(1-\pi))}}$. Also

$$
C_{p}-x^{2}=\frac{C_{p}^{2} o_{p}(1)+C_{p} \frac{\left(n \pm\left(n_{1}-n_{2}\right)\right) p}{n_{1} n_{2}}\left(1+o_{p}(1)\right)+\frac{p^{2}\left(n_{1}-n_{2}\right)^{2}}{n_{1}^{2} n_{2}^{2}}}{C_{p}\left(1+o_{p}(1)\right)+\frac{n p}{n_{1} n_{2}}\left(1+o_{p}(1)\right)} \rightarrow \infty
$$

Thus we have

$$
\frac{\Phi\left(-\frac{\sqrt{C_{p}}}{2}\right)}{\Phi\left(-\frac{x}{2}\right)} \rightarrow 0
$$

As a result

$$
\frac{W\left(\hat{\delta}_{\hat{\boldsymbol{\mu}}}\right)}{W_{O P T}} \rightarrow \infty
$$

(ii) While $\frac{C_{p}}{p / n} \rightarrow c$ with $0<c<\infty$

$$
\begin{aligned}
& \frac{ \pm C_{p}\left(1+o_{p}(1)\right)+\frac{p}{n_{1} n_{2}}\left(n_{1}-n_{2}\right)\left(1+o_{p}(1)\right)}{2 \sqrt{C_{p}\left(1+o_{p}(1)\right)+\frac{n p}{n_{1} n_{2}}\left(1+o_{p}(1)\right)}} \\
= & \frac{ \pm C_{p}\left(1 \pm \frac{p}{n_{1} n_{2} C_{p}}\left(n_{1}-n_{2}\right)\left(1+o_{p}(1)\right)\right)}{2 \sqrt{C_{p}\left(1+\frac{n p}{n_{1} n_{2} C_{p}}\left(1+o_{p}(1)\right)\right.}} \\
= & \frac{ \pm \sqrt{C_{p}}\left(1 \pm \frac{p}{n_{1} n_{2} C_{p}}\left(n_{1}-n_{2}\right)\left(1+o_{p}(1)\right)\right)}{2 \sqrt{\left(1+\frac{n p}{n_{1} n_{2} C_{p}}\left(1+o_{p}(1)\right)\right.}} \\
\rightarrow & \frac{ \pm \sqrt{C_{0}}\left(1 \pm \frac{1}{c} \frac{2 \pi-1}{\pi(1-\pi)}\right)}{2 \sqrt{1+\frac{1}{c} \frac{1}{\pi(1-\pi)}}}
\end{aligned}
$$

Since $C_{p} \rightarrow C_{0}$,

$$
W_{1}\left(\hat{\delta}_{\hat{\boldsymbol{\mu}}}\right) \rightarrow 1-\Phi\left(\frac{\sqrt{C_{0}}\left(1+\frac{1}{c} \frac{2 \pi-1}{\pi(1-\pi)}\right)}{2 \sqrt{1+\frac{1}{c} \frac{1}{\pi(1-\pi)}}}\right)
$$


and

$$
W_{2}\left(\hat{\delta}_{\hat{\boldsymbol{\mu}}}\right) \rightarrow 1-\Phi\left(\frac{\sqrt{C_{0}}\left(1-\frac{1}{c} \frac{2 \pi-1}{\pi(1-\pi)}\right)}{2 \sqrt{1+\frac{1}{c} \frac{1}{\pi(1-\pi)}}}\right)
$$

If $\frac{p}{n} \rightarrow C$ with $0<C<\infty$, then $0<C_{0}<\infty$. Since $\Phi(x)$ is convex function in the sense that $\frac{1}{2}(\Phi(x+\epsilon)+\Phi(x-\epsilon)) \leq \Phi(x)$ for any $x>0$ and $x>\epsilon>0$,

$$
\begin{aligned}
\lim _{P} W\left(\hat{\delta}_{\hat{\boldsymbol{\mu}}}\right) & =\lim _{P} \frac{1}{2}\left(W_{1}\left(\hat{\delta}_{\hat{\boldsymbol{\mu}}}\right)+W_{2}\left(\hat{\delta}_{\hat{\boldsymbol{\mu}}}\right)\right) \\
& \geq 1-\Phi\left(\frac{\sqrt{C_{0}}}{2 \sqrt{1+\frac{1}{c} \frac{1}{\pi(1-\pi)}}}\right)>1-\Phi\left(\frac{\sqrt{C_{0}}}{2}\right)
\end{aligned}
$$

where $\lim _{P}$ means converge in probability with $p \rightarrow \infty$ and $n \rightarrow \infty$.

If $\frac{p}{n} \rightarrow \infty$, then $C_{0}=\infty$. Hence $W\left(\hat{\delta}_{\hat{\boldsymbol{\mu}}}\right)=\frac{1}{2}\left(W_{1}\left(\hat{\delta}_{\hat{\boldsymbol{\mu}}}\right)+W_{2}\left(\hat{\delta}_{\hat{\boldsymbol{\mu}}}\right)\right) \rightarrow 0$. By similar argument in (i), we have $\frac{W\left(\hat{\delta}_{\hat{\mu}}\right)}{W_{O P T}} \rightarrow \infty$.

(iii) While $\frac{C_{p}}{p / n} \rightarrow 0$,

$$
\begin{aligned}
& \frac{ \pm C_{p}\left(1+o_{p}(1)\right)+\frac{p}{n_{1} n_{2}}\left(n_{1}-n_{2}\right)\left(1+o_{p}(1)\right)}{2 \sqrt{C_{p}\left(1+o_{p}(1)\right)+\frac{n p}{n_{1} n_{2}}\left(1+o_{p}(1)\right)}} \\
&= \frac{\sqrt{\frac{p}{n}}\left( \pm C_{p} /\left(\frac{p}{n}\right)+\frac{n\left(n_{1}-n_{2}\right)}{n_{1} n_{2}}\left(1+o_{p}(1)\right)\right)}{2 \sqrt{\left(C_{p} /\left(\frac{p}{n}\right)+\frac{n^{2}}{n_{1} n_{2}}\left(1+o_{p}(1)\right)\right.}} \\
& \rightarrow \begin{cases}\infty & \text { if } n_{1}>n_{2}, \\
-\infty & \text { if } n_{1}<n_{2} .\end{cases}
\end{aligned}
$$

Which yields $W\left(\hat{\delta}_{\hat{\boldsymbol{\mu}}}\right) \rightarrow \frac{1}{2}$.

Proof of Corollary 1. Noticing that when $n_{1}=n_{2}=n / 2, \hat{\boldsymbol{\epsilon}}_{1} \sim N\left(0, \frac{1}{n_{1}} \Sigma\right)$ and $\hat{\boldsymbol{\epsilon}}_{2} \sim N\left(0, \frac{1}{n_{1}} \Sigma\right)$. Let $\tilde{\epsilon}_{i}=\sqrt{n_{i}} \Sigma^{\frac{1}{2}} \hat{\epsilon}_{i}$ for $i=1,2$. Then $\tilde{\epsilon}_{i} \sim N\left(0, I_{p}\right)$ and

$$
E\left(\hat{\boldsymbol{\epsilon}}_{1}^{T} \Sigma^{-1} \hat{\boldsymbol{\epsilon}}_{1}-\hat{\boldsymbol{\epsilon}}_{2}^{T} \Sigma^{-1} \hat{\boldsymbol{\epsilon}}_{2}\right)^{2}=E\left(\tilde{\epsilon}_{1}^{T} \tilde{\epsilon}_{1}-\tilde{\epsilon}_{2}^{T} \tilde{\epsilon}_{2}\right)^{2} / N_{1}^{2}=\sum_{j=1}^{p}\left(\tilde{\epsilon}_{1 j}^{2}-\tilde{\epsilon}_{2 j}^{2}\right)^{2} / n_{1}^{2}=6 p / n_{1}^{2}
$$

Hence we have:

$$
\hat{\boldsymbol{\epsilon}}_{1}^{T} \Sigma^{-1} \hat{\boldsymbol{\epsilon}}_{1}-\hat{\boldsymbol{\epsilon}}_{2}^{T} \Sigma^{-1} \hat{\boldsymbol{\epsilon}}_{2}=O_{p}\left(\frac{\sqrt{p}}{n}\right)
$$

Similar to the proof of (A.39) we have:

$$
W_{1}\left(\hat{\delta}_{\hat{\boldsymbol{\mu}}}\right) \leq 1-\Phi\left(\frac{C_{p}\left(1+o_{p}(1)\right)+\frac{\sqrt{p}}{n}\left(1+o_{p}(1)\right)}{2 \sqrt{C_{p}\left(1+o_{p}(1)\right)+\frac{4 p}{n}\left(1+o_{p}(1)\right)}}\right)
$$


and

$$
\begin{gathered}
W_{2}(\hat{\boldsymbol{\mu}}) \leq \Phi\left(\frac{-C_{p}\left(1+o_{p}(1)\right)+\frac{\sqrt{p}}{n}\left(1+o_{p}(1)\right)}{2 \sqrt{C_{p}\left(1+o_{p}(1)\right)+\frac{4 p}{n}\left(1+o_{p}(1)\right)}}\right) \\
\frac{ \pm C_{p}\left(1+o_{p}(1)\right)+\frac{\sqrt{p}}{n}\left(1+o_{p}(1)\right)}{2 \sqrt{C_{p}\left(1+o_{p}(1)\right)+\frac{4 p}{n}\left(1+o_{p}(1)\right)}}=\frac{ \pm C_{p} / \sqrt{\frac{p}{n}}\left(1+o_{p}(1)\right)+\frac{1}{\sqrt{n}}\left(1+o_{p}(1)\right)}{2 \sqrt{\left.C_{p} / \frac{p}{n}\left(1+o_{p}(1)\right)+4+o_{p}(1)\right)}} \\
\begin{cases}\rightarrow \pm \infty & \text { if } \frac{C_{p}}{\sqrt{p / n}} \rightarrow \infty \\
\rightarrow \pm \frac{c}{4} & \text { if } \frac{C_{p}}{\sqrt{p / n}} \rightarrow c \text { and } p / n \rightarrow \infty \\
\rightarrow \pm \frac{c}{2 \sqrt{4+c / \sqrt{C}}} & \text { if } \frac{C_{p}}{\sqrt{p / n}} \rightarrow c \text { and } p / n \rightarrow C<\infty \\
\rightarrow 0 & \text { if } \frac{C_{p}}{\sqrt{p / n}} \rightarrow 0\end{cases}
\end{gathered}
$$

The proof of $\frac{W\left(\hat{\delta}_{\hat{\mu}}\right)}{W_{O P T}} \rightarrow \infty$ is the same as that in the proof of Theorem 2.3(1). This completes the proof.

\section{A.2. Proofs for consistency of one-step PMLE}

Proof of Theorem 3.1. In the algorithm, we estimate $\hat{\boldsymbol{\beta}}^{(0)}$ first. Then $\hat{\boldsymbol{\theta}}^{(0)}$ is estimated by fixing $\boldsymbol{\beta}=\hat{\boldsymbol{\beta}}^{(0)}$. Then update $\boldsymbol{\beta}=\hat{\boldsymbol{\beta}}^{(1)}$ by fixing $\boldsymbol{\theta}=\hat{\boldsymbol{\theta}}^{(0)} \cdot \boldsymbol{\theta}=\hat{\boldsymbol{\theta}}^{(1)}$ is updated in the last step by fixing $\boldsymbol{\beta}=\hat{\boldsymbol{\beta}}^{(1)}$. So the idea is to prove the theorem in the following sequence: (a) The consistency and sparsity of $\boldsymbol{\beta}^{(0)}$; (b) The consistency of $\hat{\boldsymbol{\theta}}^{(0)}$; (c) The consistency and sparsity of $\hat{\boldsymbol{\beta}}^{(1)}$; (d) The consistency of $\hat{\boldsymbol{\theta}}^{(1)}$.

(a) We first prove $\left\|\hat{\boldsymbol{\beta}}^{(0)}-\boldsymbol{\beta}_{0}\right\|_{2}=O_{p}\left(\sqrt{\frac{s}{n}}\right)$ and $\hat{\boldsymbol{\beta}}_{2}^{(0)}=0$ with probability tending to 1 , where $\hat{\boldsymbol{\beta}}_{2}^{(0)}$ is the $p-s$ dimension sub-vector of $\hat{\boldsymbol{\beta}}^{(0)}=$ $\left(\hat{\boldsymbol{\beta}}_{1}^{(0) T}, \hat{\boldsymbol{\beta}}_{2}^{(0) T}\right)^{T}$. The proof of (a) is the same as the proof of (c), except the loss function is defined as $R(\boldsymbol{\beta})$ which is negative of the penalized MLE function (3.1) with covariance matrix $\dot{\Sigma}$ replaced by $\operatorname{diag}_{n-1}\left(I_{p}\right)$. Then the parameters are estimated by minimize the loss function. We omit the proof here and illustrate the details in (c).

(b) Second, we prove $\left\|\hat{\boldsymbol{\theta}}^{(0)}-\boldsymbol{\theta}_{0}\right\|_{2}=O_{p}\left(\sqrt{\frac{1}{n p}}\right)$. Fixing $\boldsymbol{\beta}=\hat{\boldsymbol{\beta}}^{0}$, write

$$
\begin{aligned}
& F\left(\boldsymbol{\theta}, \hat{\boldsymbol{\beta}}^{(0)} ; \mathbf{Z}\right) \\
= & -\frac{n p}{2} \log (2 \pi)-\frac{1}{2} \log |\dot{\Sigma}(\boldsymbol{\theta})|-\frac{1}{2}\left(\mathbf{Z}-\boldsymbol{X} \hat{\boldsymbol{\beta}}^{(0)}\right)^{T} \dot{\Sigma}^{-1}(\boldsymbol{\theta})\left(\mathbf{Z}-\boldsymbol{X} \hat{\boldsymbol{\beta}}^{(0)}\right)
\end{aligned}
$$

It is sufficient to prove for any given $\epsilon>0$ the smallest convergence rate of $\eta_{n, p}$ is $\sqrt{\frac{1}{n p}}$ such that we have

$$
P\left(\sup _{\|\boldsymbol{u}\|_{=} C} F\left(\boldsymbol{\theta}_{0}+\boldsymbol{u} \eta_{n, p}, \hat{\boldsymbol{\beta}}^{(0)} ; \mathbf{Z}\right)<F\left(\boldsymbol{\theta}_{0}, \hat{\boldsymbol{\beta}}^{(0)} ; \mathbf{Z}\right)\right)>1-\epsilon
$$


This implies there exists a local maximum for the function $Q\left(\boldsymbol{\theta}, \hat{\boldsymbol{\beta}}^{(0)} ; \mathbf{Z}\right)$ of $\boldsymbol{\theta}$ in the neighborhood of $\boldsymbol{\theta}_{0}$ with the radius at most proportional to $\eta_{n, p}$.

By Taylor's expansion, $\dot{\Sigma}\left(\boldsymbol{\theta}_{0}+\boldsymbol{u} \eta_{n, p}\right)-\dot{\Sigma}\left(\boldsymbol{\theta}_{0}\right)=\sum_{j=1}^{q} \frac{\partial \dot{\Sigma}\left(\boldsymbol{\theta}^{*}\right)}{\partial \theta_{j}} u_{\theta_{j}} \eta_{n, p}$, where $\boldsymbol{\theta}^{*}$ is between $\boldsymbol{\theta}_{0}+\boldsymbol{u} \eta_{n, p}$ and $\boldsymbol{\theta}_{0}$. Denote $\dot{\Sigma}^{j}\left(\boldsymbol{\theta}^{*}\right)=\frac{\partial \dot{\Sigma}\left(\boldsymbol{\theta}^{*}\right)}{\partial \theta_{j}}$, then

$$
\begin{aligned}
& F\left(\boldsymbol{\theta}_{0}+\boldsymbol{u} \eta_{n, p}, \hat{\boldsymbol{\beta}}^{(0)} ; \mathbf{Z}\right)-F\left(\boldsymbol{\theta}_{0}, \hat{\boldsymbol{\beta}}^{(0)} ; \mathbf{Z}\right) \\
= & {\left[F\left(\boldsymbol{\theta}_{0}+\boldsymbol{u} \eta_{n, p}, \boldsymbol{\beta}_{0}\right)-F\left(\boldsymbol{\theta}_{0}, \boldsymbol{\beta}_{0}\right)\right] } \\
& -\sum_{j=1}^{q}\left(\mathbf{Z}-\boldsymbol{X} \boldsymbol{\beta}_{0}\right)^{T} \dot{\Sigma}^{j}\left(\boldsymbol{\theta}^{*}\right) \boldsymbol{X}\left(\hat{\boldsymbol{\beta}}^{(0)}-\boldsymbol{\beta}_{0}\right) u_{\theta_{j}} \eta_{n, p} \\
& -\frac{1}{2} \sum_{j=1}^{q}\left(\hat{\boldsymbol{\beta}}^{(0)}-\boldsymbol{\beta}_{0}\right)^{T} X^{T} \dot{\Sigma}^{j}\left(\boldsymbol{\theta}^{*}\right) X\left(\hat{\boldsymbol{\beta}}^{(0)}-\boldsymbol{\beta}_{0}\right) u_{\theta_{j}} \eta_{n, p} \\
= & (I)+(I I)+(I I I)
\end{aligned}
$$

where

$$
\begin{aligned}
(I)= & F\left(\boldsymbol{\theta}_{0}+\boldsymbol{u} \eta_{n, p}, \boldsymbol{\beta}_{0}\right)-F\left(\boldsymbol{\theta}_{0}, \boldsymbol{\beta}_{0}\right) \\
= & -\frac{n-1}{2} \boldsymbol{u}^{T} T \boldsymbol{u} \eta_{n, p}^{2}+\left(\frac{\partial F}{\partial \boldsymbol{\theta}}\left(\boldsymbol{\theta}_{0}\right)\right)^{T} \boldsymbol{u} \eta_{n, p} \\
& +\frac{1}{2} \boldsymbol{u}^{T}\left(\frac{\partial^{2} F}{\partial \boldsymbol{\theta} \partial \boldsymbol{\theta}^{T}}\left(\boldsymbol{\theta}^{*}\right)+(n-1) T\right) \boldsymbol{u} \eta_{n, p}^{2} \\
= & (1)+(2)+(3) \\
(I I)= & \sum_{j=1}^{q}\left(\hat{\boldsymbol{\beta}}^{(0)}-\boldsymbol{\beta}_{0}\right)^{T} \boldsymbol{X}^{T} \dot{\Sigma}^{j}\left(\boldsymbol{\theta}^{*}\right)\left(\mathbf{Z}-\boldsymbol{X} \boldsymbol{\beta}_{0}\right) u_{j} \eta_{n, p} \\
(I I I)= & -\frac{1}{2} \sum_{j=1}^{q}\left(\hat{\boldsymbol{\beta}}^{(0)}-\boldsymbol{\beta}_{0}\right)^{T} \boldsymbol{X}^{T} \dot{\Sigma}^{j}\left(\boldsymbol{\theta}^{*}\right) \boldsymbol{X}\left(\hat{\boldsymbol{\beta}}^{(0)}-\boldsymbol{\beta}_{0}\right) u_{j} \eta_{n, p}
\end{aligned}
$$

We consider (I) first. $T$ in (1) is $q \times q$ matrix with its $(i, j)$ th element as $t_{i j}\left(\boldsymbol{\theta}_{0}\right)$, where $t_{i j}(\boldsymbol{\theta})=\operatorname{tr}\left(\Sigma^{-1}(\boldsymbol{\theta}) \Sigma_{i}(\boldsymbol{\theta}) \Sigma^{-1}(\boldsymbol{\theta}) \Sigma_{j}(\boldsymbol{\theta})\right)$. By Assumption 4 and Assumption 5 and using the similar argument in proving the bound of $(I)$ in Theorem 2.1, there exist a constant $K$, such that

$$
(1)=-\frac{n}{2} \sum_{i, j=1}^{q} t_{i j}\left(\boldsymbol{\theta}_{0}\right) u_{i} u_{j} \eta_{n, p}^{2} \leq-K n p \eta_{n, p}^{2}\|\boldsymbol{u}\|_{2}^{2}
$$

with probability tending to 1 . In regarding to (2),

$$
\frac{\partial F}{\partial \theta_{j}}\left(\boldsymbol{\theta}_{0}\right)=\frac{n-1}{2} \operatorname{tr}\left(\Sigma^{j}\left(\boldsymbol{\theta}_{0}\right) \Sigma\left(\boldsymbol{\theta}_{0}\right)\right)-\frac{1}{2}(\mathbf{Z}-\boldsymbol{X} \boldsymbol{\beta})^{T} \dot{\Sigma}^{j}\left(\boldsymbol{\theta}_{0}\right)(\mathbf{Z}-\boldsymbol{X} \boldsymbol{\beta})
$$


Notice that $\mathbf{Z}-\boldsymbol{X} \boldsymbol{\beta} \sim N(0, \dot{\Sigma}(\boldsymbol{\theta}))$ and $\operatorname{tr}\left(\dot{\Sigma}^{j} \dot{\Sigma}\right)=(n-1) \operatorname{tr}\left(\Sigma^{j} \Sigma\right)$. By Lemma 2,

$$
\begin{aligned}
(2) & \left.=O_{p}\left(\sqrt{\operatorname{tr}\left(\dot{\Sigma} \dot{\Sigma} j \dot{\Sigma}^{j}\right.}\right) \eta_{n, p}\|\boldsymbol{u}\|_{2}\right)=O_{p}\left(\sqrt{(n-1) \operatorname{tr}\left(\sum \Sigma^{j} \Sigma^{j}\right)} \eta_{n, p}\|\boldsymbol{u}\|_{2}\right) \\
& \left.=O_{p}\left(\sqrt{(n-1)\left\|\Sigma^{j}\right\|_{F}^{2}} \eta_{n, p}\|\boldsymbol{u}\|_{2}\right)\|\boldsymbol{u}\|_{2}\right)
\end{aligned}
$$

By Assumption 3, Assumption 6, (2) $=O_{p}\left(\sqrt{n p} \eta_{n, p}\right)$.

Then we consider (3). For any $j, k=1,2, \ldots, q$

$$
\begin{aligned}
& \frac{\partial^{2}(F)}{\partial \theta_{j} \partial \theta_{k}}\left(\boldsymbol{\theta}^{*}\right) \\
= & \frac{n-1}{2}\left(\operatorname{tr}\left(\Sigma^{j k}\left(\boldsymbol{\theta}^{*}\right) \Sigma\left(\boldsymbol{\theta}^{*}\right)-t_{j k}\left(\boldsymbol{\theta}^{*}\right)\right)-\frac{1}{2}(\mathbf{Z}-\boldsymbol{X} \boldsymbol{\beta})^{T} \dot{\Sigma}^{j k}\left(\boldsymbol{\theta}^{*}\right)(\mathbf{Z}-\boldsymbol{X} \boldsymbol{\beta})\right.
\end{aligned}
$$

Thus (3) could be written as:

$$
\begin{aligned}
& (3)= \\
& \sum_{j, k=1}^{q} \frac{n-1}{2}\left(\operatorname{tr}\left(\Sigma^{j k}\left(\boldsymbol{\theta}^{*}\right) \Sigma\left(\boldsymbol{\theta}_{0}\right)\right)-\frac{1}{2}(\mathbf{Z}-\boldsymbol{X} \boldsymbol{\beta})^{T} \Sigma^{j k}\left(\boldsymbol{\theta}^{*}\right)(\mathbf{Z}-\boldsymbol{X} \boldsymbol{\beta})\right) u_{\theta_{j}} u_{\theta_{k}} \eta_{n, p}^{2} \\
& +\sum_{j, k=1}^{q} \frac{n-1}{2}\left(\operatorname{tr}\left(\Sigma^{j k}\left(\boldsymbol{\theta}^{*}\right) \Sigma\left(\boldsymbol{\theta}^{*}\right)\right)-\operatorname{tr}\left(\Sigma^{j k}\left(\boldsymbol{\theta}^{*}\right) \Sigma\left(\boldsymbol{\theta}_{0}\right)\right)\right) u_{\theta_{j}} u_{\theta_{k}} \eta_{n, p}^{2} \\
& +\sum_{j, k=1}^{q} \frac{n-1}{2}\left(t_{j k}\left(\boldsymbol{\theta}_{0}\right)-t_{j k}\left(\boldsymbol{\theta}^{*}\right)\right) u_{\theta_{j}} u_{\theta_{k}} \eta_{n, p}^{2} \\
& =(i)+(i i)+(i i i)
\end{aligned}
$$

By Lemma 2 and Assumption 6, $(i)=O_{p}\left(\sqrt{(n-1) \operatorname{tr}\left(\Sigma \Sigma^{j k} \Sigma^{j k}\right)} \eta_{n, p}^{2}\right)=$ $O_{p}\left(\sqrt{n p} \eta_{n, p}^{2}\right)$. Similar to the deriving the order of (5) and (6) in the proof of Theorem 2.1, $(i i)=O_{p}\left(n \sqrt{p^{3}} \eta_{n, p}^{3}\right)$ and $(i i i)=O_{p}\left(n p \eta_{n, p}^{3}\right)$. By choosing large $C=\|\boldsymbol{u}\|_{2}$, the minimal rate that (2) and (3) are dominated by (1) is $\eta_{n, p}=O_{p}\left(\frac{1}{\sqrt{n p}}\right)$.

Now we consider $(I I)$. Denote $B=\sum_{i=1}^{n-1} \boldsymbol{X}_{i}$. Then by Lemma 1 , for any $j=1,2, \ldots, q$,

$$
\begin{aligned}
& \left(\hat{\boldsymbol{\beta}}^{(0)}-\boldsymbol{\beta}_{0}\right)^{T} \boldsymbol{X}^{T} \dot{\Sigma}^{j}\left(\boldsymbol{\theta}^{*}\right)\left(\mathbf{Z}-\boldsymbol{X} \boldsymbol{\beta}_{0}\right) \\
= & O_{p}\left(\sqrt{\operatorname{tr}\left(\boldsymbol{X}^{T} \dot{\Sigma} j\left(\boldsymbol{\theta}^{*}\right) \dot{\Sigma}\left(\boldsymbol{\theta}^{*}\right) \dot{\Sigma^{j}}\left(\boldsymbol{\theta}^{*}\right) \boldsymbol{X}\right)}\left\|\hat{\boldsymbol{\beta}}^{(0)}-\boldsymbol{\beta}_{0}\right\|_{2}\right) \\
= & O_{p}\left(\sqrt{\operatorname{tr}\left(\sum_{i=1}^{n-1} \boldsymbol{X}_{j}^{T} \Sigma^{j *} \Sigma^{*} \Sigma^{j *} \boldsymbol{X}_{i}\right)+\operatorname{tr}\left(B^{T} \Sigma^{j *} \Sigma^{*} \Sigma^{j *} B\right)}\left\|\hat{\boldsymbol{\beta}}^{(0)}-\boldsymbol{\beta}_{0}\right\|_{2}\right)
\end{aligned}
$$

where $\Sigma^{*}=\Sigma\left(\boldsymbol{\theta}^{*}\right)$ and $\Sigma^{j *}=\Sigma^{j}\left(\boldsymbol{\theta}^{*}\right)$. Notice that $\sum_{i=1}^{n-1} \boldsymbol{X}_{i}^{T} \boldsymbol{X}_{i}=\left(\frac{n_{1} n_{2}}{n}-\right.$ 
$\left.\frac{n_{1}^{2}}{n^{2}}\right) I_{p \times p}$ and $\left(\sum_{i=1}^{n-1} \boldsymbol{X}_{i}\right)^{T}\left(\sum_{i=1}^{n-1} \boldsymbol{X}_{i}\right)=\frac{n_{1}^{2}}{n^{2}} I_{p \times p}$. Then by Assumption 6

$$
\begin{aligned}
& \operatorname{tr}\left(\sum_{i=1}^{n-1} \boldsymbol{X}_{j}^{T} \Sigma^{j *} \Sigma^{*} \Sigma^{j *} \boldsymbol{X}_{i}\right) \\
= & \left(\frac{n_{1} n_{2}}{n}-\frac{n_{1}^{2}}{n_{2}}\right) \operatorname{tr}\left(\Sigma^{j *} \Sigma^{*} \Sigma^{j *}\right) \leq \lambda_{\max }(\Sigma) \frac{n_{1} n_{2}}{n}\left\|\Sigma^{j *}\right\|_{F}^{2}=O_{p}(n p) .
\end{aligned}
$$

Similarly $\operatorname{tr}\left(B^{T} \Sigma^{j *} \Sigma^{*} \Sigma^{j *} B\right)=O_{p}(n p)$.

Since $\left\|\boldsymbol{\beta}^{(0)}-\boldsymbol{\beta}_{0}\right\|_{2}=O_{p}\left(\sqrt{\frac{s}{n}}\right),(I I)=O_{p}\left(\sqrt{p s} \eta_{n, p}\right)$

For (III), by Assumption 6 , for any $j=1,2, \ldots, q$ we also have:

$$
\begin{aligned}
& \left(\hat{\boldsymbol{\beta}}^{(0)}-\boldsymbol{\beta}_{0}\right)^{T} \boldsymbol{X}^{T} \dot{\Sigma}^{j}\left(\boldsymbol{\theta}^{*}\right) \boldsymbol{X}\left(\hat{\boldsymbol{\beta}}^{(0)}-\boldsymbol{\beta}_{0}\right) \\
= & \left(\hat{\boldsymbol{\beta}}^{(0)}-\boldsymbol{\beta}_{0}\right)^{T} \boldsymbol{X}^{T} \operatorname{diag}_{n-1} \Sigma^{j}\left(\boldsymbol{\theta}^{*}\right)\left(\tilde{I}_{n-1, p}+\tilde{J}_{n-1, p}\right) \boldsymbol{X}\left(\hat{\boldsymbol{\beta}}^{(0)}-\boldsymbol{\beta}_{0}\right) \\
\leq & \lambda_{\max }\left(\Sigma^{j *}\right)\left(\left(\hat{\boldsymbol{\beta}}^{(0)}-\boldsymbol{\beta}_{0}\right)^{T}\left(\sum_{i=1}^{n-1} \boldsymbol{X}_{i}^{T} \boldsymbol{X}_{i}+\left(\sum_{i=1}^{n-1} \boldsymbol{X}_{i}\right)^{T}\left(\sum_{i=1}^{n-1} \boldsymbol{X}_{i}\right)\right)\left(\hat{\boldsymbol{\beta}}^{(0)}-\boldsymbol{\beta}_{0}\right)\right) \\
= & O_{p}\left(n\left\|\hat{\boldsymbol{\beta}}^{(0)}-\boldsymbol{\beta}_{0}\right\|_{2}^{2}\right)=O_{p}(s)
\end{aligned}
$$

Thus $(I I I)=O_{p}\left(s \eta_{n, p}\right)$. Both $(I I)$ and $(I I I)$ are dominated by $(I)$ while $\eta_{n, p}=O_{p}\left(\sqrt{\frac{1}{n p}}\right)$. This concludes the proof of $\left\|\hat{\boldsymbol{\theta}}^{(0)}-\boldsymbol{\theta}_{0}\right\|_{2}=O_{p}\left(\sqrt{\frac{1}{n p}}\right)$.

(c) Write $\hat{\boldsymbol{\beta}}^{(1)}=\left(\hat{\boldsymbol{\beta}}_{1}^{(1)}, \hat{\boldsymbol{\beta}}_{2}^{(2)}\right)^{T}$. Then we prove $\left\|\hat{\boldsymbol{\beta}}^{(1)}-\boldsymbol{\beta}_{0}\right\|_{2}=O_{p}\left(\sqrt{\frac{s}{n}}\right)$ and $\hat{\boldsymbol{\beta}}_{2}^{(1)}=0$ with probability tending to 1 , where $\hat{\boldsymbol{\beta}}_{(1)}^{1}$ formed by elements in $\operatorname{supp}\left(\boldsymbol{\beta}_{0}\right)$ and $\hat{\boldsymbol{\beta}}_{2}^{(1)}$ is a $p-s$ sub-vector of $\hat{\boldsymbol{\beta}}^{(1)}$. Let $\eta_{n, p}=$ $O_{p}\left(\left\|\hat{\boldsymbol{\theta}}^{(0)}-\boldsymbol{\theta}_{0}\right\|_{2}\right)=O_{p}\left(\sqrt{\frac{1}{n p}}\right)$. We use two steps to prove the consistency and sparsity.

step 1. We first prove consistency on s-dimensional space. Define the loglikelihood function for $\boldsymbol{\beta}^{1}$ as

$$
\begin{aligned}
\bar{Q}\left(\hat{\boldsymbol{\theta}}^{(0)}, \boldsymbol{\beta}^{1}\right)= & -\frac{n p}{2} \log (2 \pi)-\frac{1}{2} \log \left|\dot{\Sigma}\left(\hat{\boldsymbol{\theta}}^{(0)}\right)\right| \\
& -\frac{1}{2}\left(\mathbf{Z}-\boldsymbol{X}^{1} \boldsymbol{\beta}^{1}\right)^{T} \dot{\Sigma}^{-1}\left(\hat{\boldsymbol{\theta}}^{(0)}\right)\left(\mathbf{Z}-\boldsymbol{X}^{1} \boldsymbol{\beta}^{1}\right)-n \sum_{j=1}^{n} P_{\lambda}\left(\left|\beta_{j}\right|\right)
\end{aligned}
$$

where $\boldsymbol{\beta}^{1}$ is sub-vector of $\boldsymbol{\beta}_{0}=\left(\boldsymbol{\beta}^{1 T}, \boldsymbol{\beta}^{2 T}\right)^{T}$ formed by elements in supp $\boldsymbol{\beta}_{0}$. We first $\left\|\hat{\boldsymbol{\beta}}_{1}^{(1)}-\boldsymbol{\beta}^{1}\right\|_{2}=O_{p}\left(\sqrt{\frac{s}{n}}\right)$. It is sufficient to prove that for any $\epsilon>0$, the smallest rate of $\xi_{n, p}$ is $\sqrt{\frac{s}{n}}$ such that we have:

$$
P\left(\sup _{\|\boldsymbol{u}\|_{2}=C} \bar{Q}\left(\hat{\boldsymbol{\theta}}^{(0)}, \boldsymbol{\beta}^{1}+u \xi_{n, p}\right)<\bar{Q}\left(\hat{\boldsymbol{\theta}}^{(0)}, \boldsymbol{\beta}^{1}\right)\right)>1-\epsilon
$$


where $\boldsymbol{u} \in \mathbb{R}^{s}$. This implies that with probability tending to 1 , there is a local maximizer $\hat{\boldsymbol{\beta}}_{1}^{(1)}$ of the function $\bar{Q}$ in the neighborhood of $\boldsymbol{\beta}_{0}^{1}$ with the radius of $\boldsymbol{\beta}_{0}^{1}$ at most proportional to $\xi_{n, p}$.

$$
\begin{aligned}
& \bar{Q}\left(\hat{\boldsymbol{\theta}}^{(0)}, \boldsymbol{\beta}_{0}^{1}+u \xi_{n, p}\right)-\bar{Q}\left(\hat{\boldsymbol{\theta}}^{(0)}, \boldsymbol{\beta}_{0}^{1}\right) \\
= & -\frac{1}{2} \boldsymbol{u}^{T} \boldsymbol{X}^{1 T} \dot{\Sigma}^{-1}\left(\hat{\boldsymbol{\theta}}^{(0)}\right) \boldsymbol{X}^{1} \boldsymbol{u} \xi_{n, p}^{2}-\left(\mathbf{Z}-\boldsymbol{X}^{1} \boldsymbol{\beta}_{0}^{1}\right) \dot{\Sigma}^{-1}\left(\hat{\boldsymbol{\theta}}^{(0)}\right) \boldsymbol{X}^{1} \boldsymbol{u} \xi_{n, p} \\
& \left.-n p \sum_{j=1}^{m}\left(p_{\lambda_{n, p}}^{\prime}\left(\left|\beta_{0 j}\right|\right) \operatorname{sgn}\left(\beta_{j}\right) u_{\beta_{j} \xi_{n, p}}+p_{\lambda_{n, p}}^{\prime \prime}\left(\left|\beta_{0 j}\right|\right) u_{\beta_{j}}^{2} \xi_{n}^{2}\right)(1+o(1))\right) \\
= & (I)+(I I)+(I I I)
\end{aligned}
$$

By Taylor's expansion, $\dot{\Sigma}^{-1}\left(\hat{\boldsymbol{\theta}}^{(0)}\right)=\dot{\Sigma}^{-1}\left(\boldsymbol{\theta}_{0}\right)+\sum_{j=1}^{q} \dot{\Sigma}^{j}(\boldsymbol{\theta} *)$, where $\boldsymbol{\theta} *$ is a $q$ dimension vector between $\boldsymbol{\theta}_{0}$ and $\hat{\boldsymbol{\theta}}^{(0)}$. Therefore,

$$
\begin{aligned}
(I) & =-\frac{1}{2} \boldsymbol{u}^{T} \boldsymbol{X}^{1 T} \dot{\Sigma}^{-1}\left(\boldsymbol{\theta}_{0}\right) \boldsymbol{X}^{1} \boldsymbol{u} \xi_{n, p}^{2}-\frac{1}{2} \sum_{j=1}^{q} \boldsymbol{u}^{T} \boldsymbol{X}^{1 T} \dot{\Sigma}^{j}\left(\boldsymbol{\theta}^{*}\right) \boldsymbol{X}^{1} \boldsymbol{u} \xi_{n, p}^{2} u_{j} \eta_{n, p} \\
& =(1)+(2)
\end{aligned}
$$

and

$$
\begin{aligned}
& (I I)= \\
& -\left(\mathbf{Z}-\boldsymbol{X}^{1} \boldsymbol{\beta}_{0}^{1}\right) \dot{\Sigma}^{-1}\left(\hat{\boldsymbol{\theta}}^{(0)}\right) \boldsymbol{X}^{1} \boldsymbol{u} \xi_{n, p}+\sum_{j=1}^{q}\left(\mathbf{Z}-\boldsymbol{X}^{1} \boldsymbol{\beta}_{0}^{1}\right) \dot{\Sigma}^{j}\left(\boldsymbol{\theta}^{*}\right) \boldsymbol{X}^{1} \boldsymbol{u} \xi_{n, p} u_{j} \eta_{n, p} \\
= & (3)+(4)
\end{aligned}
$$

Since $\lambda_{\min }\left(\Sigma^{-1}\right)>0$ and notice $\sum_{i=1}^{n-1} \boldsymbol{X}_{i}^{1 T} \boldsymbol{X}_{i}^{1}=\left(\frac{n_{1} n_{2}}{n}-\frac{n_{1}^{2}}{n^{2}}\right) I_{s}$,

$$
\begin{aligned}
\left(\sum_{i=1}^{n-1} \boldsymbol{X}_{i=1}^{1 T}\right)\left(\sum_{i=1}^{n-1} \boldsymbol{X}_{i=1}^{1}\right)=\frac{n_{1}^{2}}{n^{2}} I_{s} \\
(1)=\frac{1}{2} \boldsymbol{u}^{T} \boldsymbol{X}^{1 T} \operatorname{diag}_{n-1}\left(\Sigma^{-1}\right)\left(\tilde{I}_{n-1, p}+\tilde{J}_{n-1, p}\right) \boldsymbol{X}^{1} \boldsymbol{u} \xi_{n, p}^{2} \\
\quad=-\frac{1}{2} \boldsymbol{u}^{T} \sum_{i=1}^{n-1} \boldsymbol{X}_{i}^{1 T} \Sigma^{-1} \boldsymbol{X}_{i}^{1} \boldsymbol{u} \xi_{n, p}^{2}-\frac{1}{2} \boldsymbol{u}^{T} \sum_{i=1}^{n-1} \boldsymbol{X}_{i}^{1 T} \Sigma^{-1} \sum_{i=1}^{n-1} \boldsymbol{X}_{i}^{1} \boldsymbol{u} \xi_{n, p}^{2} \\
\quad \leq-\frac{1}{2} \boldsymbol{u}^{T}\left(\sum_{i=1}^{n-1} \boldsymbol{X}_{i}^{1 T} \boldsymbol{X}_{i}^{1}+\sum_{i=1}^{n-1} \boldsymbol{X}_{i}^{1 T} \sum_{i=1}^{n-1} \boldsymbol{X}_{i}^{1}\right) \boldsymbol{u} \xi_{n, p}^{2} \lambda_{\min }\left(\Sigma^{-1}\right) \\
=-\frac{1}{2} \frac{n_{1} n_{2}}{n}\|\boldsymbol{u}\|_{2}^{2} \xi_{n, p}^{2} \lambda_{\min }\left(\Sigma^{-1}\right) \\
\quad \leq-\frac{1}{2} \frac{\pi(1-\pi)}{\lambda_{\max }(\Sigma)} n \xi_{n, p}^{2}\|\boldsymbol{u}\|_{2}^{2}
\end{aligned}
$$

By similar argument and Assumption 6, (2) $=O_{p}\left(n \xi_{n, p}^{2} \eta_{n, p}\right)=o_{p}((1))$ while $\eta_{n, p}=O_{p}\left(\sqrt{\frac{1}{n p}}\right)$. For (II), by Lemma 1 and Assumption 3,

$$
(3)=O_{p}\left(\sqrt{\operatorname{tr}\left(\boldsymbol{X}^{1} \dot{\Sigma}^{-1}\left(\boldsymbol{\theta}_{0}\right) \boldsymbol{X}^{1}\right)}\|\boldsymbol{u}\|_{2} \xi_{n, p}\right)
$$




$$
\begin{aligned}
& =O_{p}\left(\sqrt{\lambda_{\max }(\Sigma) \frac{n_{1} n_{2}}{n} \operatorname{tr}\left(I_{s \times s}\right)}\|\boldsymbol{u}\|_{2} \xi_{n, p}\right) \\
& \left.=O_{p}\left(\sqrt{n s}\|\boldsymbol{u}\|_{2} \xi_{n, p}\right)\right)
\end{aligned}
$$

Similarly, (4) $=O_{p}\left(\sqrt{n s}\|\boldsymbol{u}\|_{2} \xi_{n, p} \eta_{n, p}\right)=o_{p}((3))$. So we have $(I I)=$ $\left.O_{p}\left(\sqrt{n s}\|\boldsymbol{u}\|_{2} \xi_{n, p}\right)\right)$.

$(I I I)=(5)+(6)$, where

$$
\begin{aligned}
& (5)=-n \sum_{j=1}^{s} p_{\lambda_{n, p}}^{\prime}\left(\left|\beta_{0 j}\right|\right) \operatorname{sgn}\left(\beta_{j}\right) u_{\beta_{j}} \xi_{n, p} \\
& \left.(6)=-n \sum_{j=1}^{s} p_{\lambda_{n, p}}^{\prime \prime}\left(\left|\beta_{0 j}\right|\right) u_{\beta_{j}}^{2} \xi_{n}^{2}\right)(1+o(1))
\end{aligned}
$$

Since $a_{n, p}=O_{p}\left(\frac{1}{\sqrt{n}}\right)$ by Assumption 7,

$$
|(5)| \leq n \sqrt{s} a_{n, p}\|\boldsymbol{u}\|_{2}=O_{p}\left(\sqrt{n s} \xi_{n, p}\|\boldsymbol{u}\|_{2}\right)
$$

By Assumption 8

$$
\begin{aligned}
|(6)| & \leq 2 n \xi_{n, p}^{2} \sum_{j=1}^{s} p^{\prime \prime}\left(\beta_{0 j}\right) u_{\beta_{j}}^{2} \leq 2 n \xi_{n, p}^{2} b_{n, p}\|\boldsymbol{u}\|_{2}^{2} \\
& =o_{p}\left(n \xi_{n, p}^{2}\right)
\end{aligned}
$$

By choosing large $C=\|\boldsymbol{u}\|_{2}$, the smallest rate of $\xi_{n p}$ that $(I I)$ and (III) are dominated by $(I)$ is $\xi_{n, p}=O_{p}\left(\sqrt{\frac{s}{n}}\right)$. This completes the proof that $\left\|\hat{\boldsymbol{\beta}}_{1}^{(1)}-\boldsymbol{\beta}_{0}^{1}\right\|_{2}=O_{p}\left(\sqrt{\frac{s}{n}}\right)$.

step 2. in step 2 we prove that the vector $\hat{\boldsymbol{\beta}}=\left(\hat{\boldsymbol{\beta}}_{1}^{(1)}, 0\right)$ is a strict local maximizer on $\mathbb{R}^{d}$. It is sufficient to prove for any given $\boldsymbol{\beta} \in \mathbb{R}^{d}$ satisfying $\left\|\boldsymbol{\beta}-\boldsymbol{\beta}_{0}\right\|_{2}=O_{p}\left(\sqrt{\frac{s}{n}}\right)$, we have $Q\left(\boldsymbol{\beta}^{s}, \hat{\boldsymbol{\theta}}^{(0)}\right) \geq Q\left(\boldsymbol{\beta}, \hat{\boldsymbol{\theta}}^{(0)}\right)$, where $\boldsymbol{\beta}=\left(\boldsymbol{\beta}^{1 T}, \boldsymbol{\beta}^{2 T}\right)^{T}$ and $\boldsymbol{\beta}^{s}=\left(\boldsymbol{\beta}^{1 T}, 0^{T}\right)^{T}$.

Let $\epsilon=C \sqrt{\frac{s}{n}}$, it is sufficient to prove for $j=s+1, s+2, \ldots, p$ :

$$
\begin{gathered}
\frac{\partial Q\left(\boldsymbol{\beta}, \hat{\boldsymbol{\theta}}^{(0)}\right)}{\partial \beta_{j}}<0 \text { for } 0<\beta_{j}<\epsilon \\
\frac{\partial Q\left(\boldsymbol{\beta}, \hat{\boldsymbol{\theta}}^{(0)}\right)}{\partial \beta_{j}}>0 \text { for }-\epsilon<\beta_{j}<0 \\
\frac{\partial Q\left(\boldsymbol{\beta}, \hat{\boldsymbol{\theta}}^{(0)}\right)}{\partial \boldsymbol{\beta}_{j}}=\left(\mathbf{Z}-\boldsymbol{X} \boldsymbol{\beta}_{0}\right)^{T} \dot{\Sigma}^{-1}\left(\hat{\boldsymbol{\theta}}^{(0)}\right) \boldsymbol{X}_{j}+\sum_{l=1}^{p} \boldsymbol{X}_{l}^{T} \dot{\Sigma}^{-1}\left(\hat{\boldsymbol{\theta}}^{(0)}\right) \boldsymbol{X}_{j}\left(\beta_{l}-\beta_{0 l}\right)
\end{gathered}
$$




$$
\begin{aligned}
& -n P_{\lambda}^{\prime}\left(\left|\beta_{j}\right|\right) \operatorname{sgn}\left(\beta_{j}\right) \\
= & (I)+(I I)+(I I I)
\end{aligned}
$$

where $\boldsymbol{X}_{j}$ is the $j$ th column of $\boldsymbol{X}$.

We first consider (I). By Taylor's expansion,

$$
\begin{aligned}
(I) & =\left(\mathbf{Z}-\boldsymbol{X} \boldsymbol{\beta}_{0}\right)^{T} \dot{\Sigma}^{-1}\left(\boldsymbol{\theta}_{0}\right) \boldsymbol{X}_{j}+\sum_{k=1}^{q}\left(\mathbf{Z}-\boldsymbol{X} \boldsymbol{\beta}_{0}\right)^{T} \dot{\Sigma}^{k}\left(\boldsymbol{\theta}^{*}\right) \boldsymbol{X}_{j} u_{k} \eta_{n, p} \\
& =(5)+(6)
\end{aligned}
$$

Notice $\sum_{i=1}^{n-1} \boldsymbol{X}_{i j}^{T} \boldsymbol{X}_{i j}=\frac{n_{1} n_{2}}{n}-\frac{n_{1}^{2}}{n^{2}}$ and $\left(\sum_{i=1}^{n-1} \boldsymbol{X}_{i j}\right)^{T}\left(\sum_{i=1}^{n-1} \boldsymbol{X}_{i j}\right)=\frac{n_{1}^{2}}{n^{2}}$.

$$
\begin{aligned}
& (5)=\left(\mathbf{Z}-\boldsymbol{X} \boldsymbol{\beta}_{0}\right)^{T} \operatorname{diag}_{n-1}\left(\Sigma^{-1}\right)\left(\tilde{I}_{n-1, p}+\tilde{J}_{n-1, p}\right) \boldsymbol{X}_{j}=O_{p}\left(\sqrt{\boldsymbol{X}_{j} \dot{\Sigma}^{-1} \boldsymbol{X}_{j}}\right) \\
& =O_{p}\left(\sqrt{\sum_{i=1}^{n-1} \boldsymbol{X}_{i j}^{T} \Sigma^{-1} \boldsymbol{X}_{i j}+\sum_{i=1}^{n-1} \boldsymbol{X}_{i j}^{T} \Sigma^{-1} \sum_{i=1}^{n-1} \boldsymbol{X}_{i j}}\right)
\end{aligned}
$$

where $\boldsymbol{X}_{i j}$ is the $j$ th column of $\boldsymbol{X}_{i}$.

Noticing $\sum_{i=1}^{n-1} \boldsymbol{X}_{i j}^{T} \boldsymbol{X}_{i j}=\frac{n_{1} n_{2}}{n}-\frac{n_{1}^{2}}{n^{2}},\left(\sum_{i=1}^{n-1} \boldsymbol{X}_{i j}\right)^{T}\left(\sum_{i=1}^{n-1} \boldsymbol{X}_{i j}\right)=\frac{n_{1}^{2}}{n^{2}}$ and $\lambda_{\max }\left(\Sigma^{-1}\right) \leq \infty$, we have $(5)=O_{p}(\sqrt{n})$. Similarly, $(6)=O_{p}\left(\sqrt{n} \eta_{n, p}\right)=$ $o_{p}((3))$, which is dominated by (5) if $\eta_{n, p}=o(1)$.

For (II), by Taylor's expansion,

$$
\begin{aligned}
& (I I)= \\
& \sum_{l=1}^{p} \boldsymbol{X}_{l}^{T} \dot{\Sigma}^{-1}\left(\theta_{0}\right) \boldsymbol{X}_{j}\left(\beta_{l}-\beta_{0 l}\right)+\sum_{k=1}^{q} \sum_{l=1}^{p} \boldsymbol{X}_{l}^{T} \dot{\Sigma}^{k}\left(\theta^{*}\right) \boldsymbol{X}_{j}\left(\beta_{l}-\beta_{0 l}\right)\left(\theta_{k}^{*}-\theta_{0 k}\right) \\
& =(7)+(8) \\
& (7)=\sum_{l=1}^{p} \boldsymbol{X}_{l}^{T} \operatorname{diag}_{n-1}\left(\Sigma^{-1}\right)\left(\tilde{I}_{n-1, p}+\tilde{J}_{n-1, p}\right) \boldsymbol{X}_{j}\left(\beta_{l}-\beta_{0 l}\right) \\
& \quad=\sum_{l=1}^{p}\left(\sum_{i=1}^{n-1} \boldsymbol{X}_{i l}^{T} \Sigma^{-1} \boldsymbol{X}_{i j}+\sum_{i=1}^{n-1} \boldsymbol{X}_{i l}^{T} \Sigma^{-1} \sum_{i=1}^{n-1} \boldsymbol{X}_{i j}\right)\left(\beta_{l}-\beta_{0 l}\right)
\end{aligned}
$$

Notice

$$
\begin{aligned}
& \left.\left.\sum_{i=1}^{n-1} \boldsymbol{X}_{i l}^{T} \Sigma^{-1} \boldsymbol{X}_{i j} \leq \sum_{i=1}^{n-1}\left(\boldsymbol{X}_{i l}^{T} \Sigma^{-1} \boldsymbol{X}_{i l}\right)\right)^{\frac{1}{2}}\left(\boldsymbol{X}_{i j}^{T} \Sigma^{-1} \boldsymbol{X}_{i j}\right)\right)^{\frac{1}{2}} \\
\leq & \left.\left.\sum_{i=1}^{n-1} \lambda_{\max }\left(\Sigma^{-1}\right)\left(\boldsymbol{X}_{i l}^{T} \boldsymbol{X}_{i l}\right)\right)^{\frac{1}{2}}\left(\boldsymbol{X}_{i j}^{T} \boldsymbol{X}_{i j}\right)\right)^{\frac{1}{2}} \\
= & \lambda_{\max }\left(\Sigma^{-1}\right)\left(\frac{n_{1} n_{2}}{n}-\frac{n_{1}^{2}}{n^{2}}\right)=O_{p}(n) .
\end{aligned}
$$


Also, let $B_{l}=\sum_{i=1}^{n-1} \boldsymbol{X}_{i l}$. Then $B_{l}^{T} B_{l}=\left(\frac{n_{1}}{n}\right)^{2}$.

$$
\begin{aligned}
\sum_{i=1}^{n-1} \boldsymbol{X}_{i l}^{T} \Sigma^{-1} \sum_{i=1}^{n-1} \boldsymbol{X}_{i j} & =B_{l}^{T} \Sigma^{-1} B_{j} \\
& \leq\left(B_{l}^{T} \Sigma^{-1} B_{l}\right)^{1 / 2}\left(B_{j}^{T} \Sigma^{-1} B_{j}\right)^{1 / 2} \leq \lambda_{\max }\left(\Sigma^{-1}\right) \frac{n_{1}^{2}}{n^{2}}
\end{aligned}
$$

Then

$$
\text { (7) }=O_{p}\left(n\left\|\boldsymbol{\beta}-\boldsymbol{\beta}_{0}\right\|_{,}=O_{P}(\sqrt{n s})\right.
$$

Similarly, $(8)=O_{p}\left(\sqrt{n s} \eta_{n, p}\right)$ Thus

$$
\left.\frac{\partial Q(\boldsymbol{\beta})}{\partial \beta_{j}}=n \lambda_{n, p}\left(O_{P}\left(\frac{\sqrt{s}}{\sqrt{n} \lambda_{n, p}}\right)\right)+\frac{P_{\lambda_{n, p}}^{\prime}\left(\left|\beta_{j}\right|\right)}{\lambda_{n, p}} \operatorname{sgn}\left(\beta_{j}\right)\right)
$$

By assumption 9 and 10, the sign of (A.47) is determined by $\beta_{j}$, hence (A.44) followed. This implies $\hat{\boldsymbol{\beta}}^{(1)}$ should satisfy sparse property and completes the proof of step 2 .

(d) Lastly we prove $\left\|\hat{\boldsymbol{\theta}}^{(1)}-\boldsymbol{\theta}_{0}\right\|_{2}=O_{p}\left(\sqrt{\frac{1}{n p}}\right)$. Since $\left\|\hat{\boldsymbol{\beta}}^{(1)}-\boldsymbol{\beta}_{0}\right\|_{2}=O_{p}\left(\sqrt{\frac{s}{n}}\right)$, it is the same as the proof of (b). We omit the detail here and this completes the proof.

\section{A.3. Proofs for consistency for one-step PMLE with tappering}

Lemma 3. Assume Assumption 1, Assumption 2, Assumption 11 and Assumption 12 hold, we have:

(1) $\left\|\Sigma(\boldsymbol{\theta})-\Sigma(\boldsymbol{\theta})_{T}\right\|_{\infty}=O\left(\frac{1}{p^{\delta_{0}}}\right)$;

(2) $\left\|\Sigma_{k}(\boldsymbol{\theta})-\Sigma_{k, T}(\boldsymbol{\theta})\right\|_{\infty}=O\left(\frac{1}{p^{\delta_{0}}}\right)$;

(3) $\left\|\Sigma_{j k}(\boldsymbol{\theta})-\Sigma_{j k, T}(\boldsymbol{\theta})\right\|_{\infty}=O\left(\frac{1}{p^{\delta_{0}}}\right)$.

The matrix norm $\|\cdot\|_{\infty}$ for the $p \times p$ matrix $A=\left[a_{i j}\right]_{i, j=1}^{p}$ is defined as the maximum of row sumation, i.e. $\|A\|_{\infty}=\max _{i} \sum_{j=1}^{p}\left|a_{i j}\right|$

Proof. We show (1) in detail and omit the details for (2) and (3), as similar arguments can be applied.

$$
\left\|\Sigma(\boldsymbol{\theta})-\Sigma_{T}(\boldsymbol{\theta})\right\|_{\infty}=\max _{i} \sum_{j=1}^{p}\left|\gamma\left(h_{i j} ; \boldsymbol{\theta}\right) K_{T}\left(h_{i j}, w_{p}\right)-\gamma\left(h_{i j} ; \boldsymbol{\theta}\right)\right|
$$

where $h_{i j}=\left\|s_{i}-s_{j}\right\|_{2}$ is the distance between site $s_{i}$ and $s_{j}$. For any $i=$ $1,2, \ldots, p$,

$$
\sum_{j=1}^{p}\left|\gamma\left(h_{i j} ; \boldsymbol{\theta}\right) K_{T}\left(h_{i j}, w_{p}\right)-\gamma\left(h_{i j} ; \boldsymbol{\theta}\right)\right|
$$




$$
\begin{aligned}
& \leq \sum_{h_{i j}<w_{p}}\left|\gamma\left(h_{i j} ; \boldsymbol{\theta}\right) K_{T}\left(h_{i j}, w_{p}\right)-\gamma\left(h_{i j} ; \boldsymbol{\theta}\right)\right|+\sum_{h_{i j} \geq w_{p}} \gamma_{0}\left(\boldsymbol{\theta}, h_{i j}\right) \\
& =(I)+(I I)
\end{aligned}
$$

Let $A^{i}=\left\{j: h_{i j}>w_{p}\right\}$ and $B_{m}^{i}=\left\{j:(m-1) \delta \leq h_{i j}<m \delta\right\}$, where $\Delta$ is independent of $n$ and $p$. Then $A^{i} \subset \bigcup_{m=\left\lfloor\frac{w_{p}}{\Delta}\right\rfloor}^{\infty} B_{m}^{i}$. Let $V(R)$ be the volume of a d-dimensional baa of radius $R$. Then the volume of $B_{m}^{i}$ is $B_{m}^{i}=V(m \delta)-$ $V((m-1) \delta)=f_{d-1}(m) \delta^{d}$, where $f_{d-1}(m)$ is a polynomial function of $m$ with degree of $d-1$. By Assumption 1, the number of sites in any unit subset of $D \subset \mathbb{R}^{d}$ is bounded, say $\rho$. Let $\#\{A\}$ denote the cardinality of a discrete set $A$. Then we have $\#\left\{B_{m}^{i}\right\} \leq f_{d-1}(m) \delta^{d} \rho$. Then exist a constant $K$ such that $f_{d-1}(m) \leq K m^{d-1}$ Then

$$
\begin{aligned}
(I I)= & \sum_{h_{i j} \geq w_{p}}\left|\gamma\left(\boldsymbol{\theta}, h_{i j}\right)\right| \leq \sum_{m=\left\lfloor\frac{w_{p}}{\delta}\right\rfloor}^{\infty} \sum_{j \in B_{m}^{i}}\left|\gamma\left(\boldsymbol{\theta}, h_{i j}\right)\right| \\
& \leq K \rho \sum_{m=\left\lfloor\frac{w_{p}}{\delta}\right\rfloor}^{\infty} m^{d-1} \delta^{d} \max _{j \in B_{m}^{i}}\left|\gamma\left(\boldsymbol{\theta}, h_{i j}\right)\right| \\
& \leq K \rho \int_{w_{p}}^{\infty} x^{d-1}|\gamma(\boldsymbol{\theta}, x)| d x \leq \frac{K \rho}{w_{p}} \int_{0}^{\infty} x^{d}|\gamma(\boldsymbol{\theta}, x)| d x
\end{aligned}
$$

Let $A_{2}^{i}=\left\{j: h_{i j} \leq w_{p}\right\}$. Then $A_{2}^{i} \subset \bigcup_{m=1}^{\left\lfloor\frac{w_{p}}{\delta}\right\rfloor+1}$

$$
\begin{aligned}
(I)= & \sum_{h_{i j}<w_{p}}\left|\gamma\left(h_{i j} ; \boldsymbol{\theta}\right)-\gamma\left(h_{i j} ; \boldsymbol{\theta}\right) K_{T}\left(h_{i j}, w_{p}\right)\right| \\
& =2 \sum_{h_{i j}<w_{p}}\left|\gamma\left(h_{i j} ; \boldsymbol{\theta}\right)\right| \frac{h_{i j}}{w_{p}} \\
& \leq \frac{2}{w_{p}} \sum_{m=1}^{\left\lfloor\frac{w_{p}}{\delta}\right\rfloor+1} \sum_{j \in B_{m}^{i}} h_{i j}\left|\gamma\left(\boldsymbol{\theta}, h_{i j}\right)\right| \\
& \leq \frac{2 K \rho}{w_{p}} \sum_{m=1}^{\left\lfloor\frac{w_{p}}{\delta}\right\rfloor+1}(m \delta)^{d-1} \delta \max _{j \in B_{m}^{i}} h_{i j}\left|\gamma\left(\boldsymbol{\theta}, h_{i j}\right)\right| \\
& \leq \frac{2 K \rho}{w_{p}} \int_{0}^{\infty} x^{d}|\gamma(\boldsymbol{\theta}, x)| d x \\
& \leq \frac{2 K \rho}{w_{p}} \int_{0}^{\infty} x^{d} \gamma_{0}(\boldsymbol{\theta}, x) d x
\end{aligned}
$$

$w_{p}$ has the same order as $p^{1 / 2}$ by Assumption 11. By Assumption 12, both (I) and $(I I)=O\left(1 / p^{1 / 2}\right)$. This completes the proof.

Lemma 4. Assume Assumptions 3-6, Assumption 11, Assumption 12 hold, we have 
(a) $\lim _{p \rightarrow \infty} \lambda_{\min }\left(\Sigma_{T}\right)>0, \lim _{p \rightarrow \infty} \lambda_{\max }\left(\Sigma_{T}\right)<\infty$;

(b) There exists an open subset $\omega$ that contains the true parameter $\boldsymbol{\theta}_{0}$ such that for all $\boldsymbol{\theta}^{*} \in \omega$, we have:

(i) $-\infty<\lim _{p \rightarrow \infty} \lambda_{\min }\left(\Sigma_{T}^{k}\left(\boldsymbol{\theta}^{*}\right)\right)<\lim _{p \rightarrow \infty} \lambda_{\max }\left(\Sigma_{T}^{k}\left(\boldsymbol{\theta}^{*}\right)\right)<\infty$;

(ii) $-\infty<\lim _{p \rightarrow \infty} \lambda_{\min }\left(\Sigma_{T}^{k j}\left(\boldsymbol{\theta}^{*}\right)\right)<\lim _{p \rightarrow \infty} \lambda_{\max }\left(\Sigma_{T}^{k j}\left(\boldsymbol{\theta}^{*}\right)\right)<\infty$;

(iii) $\left|\frac{\partial t_{i j, T\left(\boldsymbol{\theta}^{*}\right)}}{\partial \theta_{k}}\right|=O(p)$ for all $k=1,2, \ldots, q$.

Proof. (a) Let $K_{T}=\left[K\left(h_{i j}, w\right)\right]_{i, j=1}^{p}$ be the tappering covariance. By eigenvalue inequalities of Schur product:

$$
\min _{1 \leq i \leq p} a_{i i} \lambda_{\min }(\Sigma) \leq \lambda\left(\Sigma \circ K_{T}\right) \leq \max _{1 \leq i \leq p} a_{i i} \lambda_{\max }(\Sigma)
$$

where $a_{i j}$ are the $(i, j)$ th entry of matrix $K_{T}$. By Assumption $3, \lambda_{\min }\left(\Sigma_{T}\right)>$ 0 and $\lim _{p \rightarrow \infty} \lambda_{\max }\left(\Sigma_{T}\right)<\infty$

(b) Since $\Sigma_{T}^{k}=\Sigma^{k} \circ K_{T}$ and $\Sigma_{T}^{k j}=\Sigma^{k j} \circ K_{T}(b)(i)$ and (b)(ii) hold by Assumption 6. For $(b)(i i i)$, since $t_{i j, T}(\boldsymbol{\theta})=\operatorname{tr}\left(\Sigma_{T}^{-1} \Sigma_{i, T} \Sigma_{T}^{-1} \Sigma_{j, T}\right)$

$$
\begin{aligned}
\frac{\partial t_{i j, T}(\boldsymbol{\theta})}{\partial \theta_{l}}= & \operatorname{tr}\left(\Sigma_{T}^{-1} \Sigma_{l, T} \Sigma_{T}^{-1} \Sigma_{i, T} \Sigma_{T}^{-1} \Sigma_{j, T}\right)+\operatorname{tr}\left(\Sigma_{T}^{-1} \Sigma_{i l, T} \Sigma_{T}^{-1} \Sigma_{j, T}\right) \\
& +\operatorname{tr}\left(\Sigma_{T}^{-1} \Sigma_{i, T} \Sigma_{T}^{-1} \Sigma_{l, T} \Sigma_{T}^{-1} \Sigma_{j, T}\right)+\operatorname{tr}\left(\Sigma_{T}^{-1} \Sigma_{i, T} \Sigma_{T}^{-1} \Sigma_{j l, T}\right) \\
= & (1)+(2)+(3)+(4)
\end{aligned}
$$

Then (1) can be written as:

$$
\begin{aligned}
& \quad \operatorname{tr}\left(\Sigma_{T}^{-1} \Sigma_{l, T} \Sigma_{T}^{-1} \Sigma_{i, T} \Sigma_{T}^{-1} \Sigma_{j, T}\right) \\
& =\operatorname{tr}\left(\left(\Sigma_{T}^{-1}-\Sigma^{-1}\right) \Sigma_{l, T} \Sigma_{T}^{-1} \Sigma_{i, T} \Sigma_{T}^{-1} \Sigma_{j, T}\right) \\
& \quad+\operatorname{tr}\left(\Sigma^{-1}\left(\Sigma_{l, T}-\Sigma_{l}\right) \Sigma_{T}^{-1} \Sigma_{i, T} \Sigma_{T}^{-1} \Sigma_{j, T}\right) \\
& \quad+\operatorname{tr}\left(\Sigma^{-1} \Sigma_{l}\left(\Sigma_{T}^{-1}-\Sigma^{-1}\right) \Sigma_{i, T} \Sigma_{T}^{-1} \Sigma_{j, T}\right) \\
& \quad+\operatorname{tr}\left(\Sigma^{-1} \Sigma_{l} \Sigma^{-1}\left(\Sigma_{i, T}-\Sigma_{i}\right) \Sigma_{T}^{-1} \Sigma_{j, T}\right) \\
& \quad+\operatorname{tr}\left(\Sigma^{-1} \Sigma_{l} \Sigma^{-1} \Sigma_{i}\left(\Sigma_{T}^{-1}-\Sigma^{-1}\right) \Sigma_{j, T}\right) \\
& \quad+\operatorname{tr}\left(\Sigma^{-1} \Sigma_{l} \Sigma^{-1} \Sigma_{i} \Sigma^{-1}\left(\Sigma_{j, T}-\Sigma_{j}\right)\right)+\operatorname{tr}\left(\Sigma^{-1} \Sigma_{l} \Sigma^{-1} \Sigma_{i} \Sigma^{-1} \Sigma_{j}\right)
\end{aligned}
$$

Define $\|\cdot\|_{s}$ for matrix A by $\|A\|_{s}=\max _{i}\left\{\left|\lambda_{i}(A)\right|, i=1,2, \ldots, p\right\}$, where $\lambda_{i}(A)$ is the $i$ th eigenvalue of matrix A. Notice

$$
\left\|\Sigma_{T}^{-1}-\Sigma^{-1}\right\|_{s} \leq\left\|\Sigma^{-1}\right\|_{s}\left\|\Sigma-\Sigma_{T}\right\|_{s}\left\|\Sigma_{T}^{-1}\right\|_{s} .
$$

Since $\lambda_{\min }\left(\Sigma^{-1}\right)=1 / \lambda_{\max }(\Sigma)>0,\left\|\Sigma^{-1}\right\|_{s} \leq \lambda_{\max }\left(\Sigma^{-1}\right)<\infty$. Also $\left\|\Sigma_{T}^{-1}\right\|_{s}<\infty,\left\|\Sigma_{j, T}\right\|_{s}<\infty$ for all $j=1,2, \ldots, q$. Hence $\left\|\Sigma_{T}^{-1}-\Sigma^{-1}\right\|_{s}=$ $O_{p}\left(p^{-\delta_{0}}\right)$. Then

$$
\left|\operatorname{tr}\left(\left(\Sigma_{T}^{-1}-\Sigma^{-1}\right) \Sigma_{l, T} \Sigma_{T}^{-1} \Sigma_{i, T} \Sigma_{T}^{-1} \Sigma_{j, T}\right)\right|
$$




$$
\begin{aligned}
& \leq p\left\|\left(\left(\Sigma_{T}^{-1}-\Sigma^{-1}\right) \Sigma_{l, T} \Sigma_{T}^{-1} \Sigma_{i, T} \Sigma_{T}^{-1} \Sigma_{j, T}\right)\right\|_{s} \\
& \leq p\left\|\left(\Sigma_{T}^{-1}-\Sigma^{-1}\right)\right\|_{s}\left\|\Sigma_{l, T}\right\|_{s}\left\|\Sigma_{T}^{-1}\right\|_{s}^{2}\left\|\Sigma_{i, T}\right\|_{s}\left\|\Sigma_{j, T}\right\|_{s} \\
& =O\left(p / p^{\delta_{0}}\right)=O\left(p^{1-\delta_{0}}\right)
\end{aligned}
$$

By similar argument, the first six terms in (1) all have the order of $O\left(p^{1-\delta_{0}}\right)$. Apply the same argument on $(2)-(4)$ we have:

$$
\frac{\partial t_{i j, T}(\boldsymbol{\theta})}{\partial \theta_{l}}=\frac{\partial t_{i j}(\boldsymbol{\theta})}{\partial \theta_{l}}+O\left(p^{1-\delta_{0}}\right)
$$

By Assumption 6, $\frac{\partial t_{i j, T}(\boldsymbol{\theta})}{\partial \theta_{l}}=O_{p}(p)$. This completes the proof.

Proof of Theorem 3.2. From Lemma 4, all regularity conditions for $\Sigma_{T}$ are satisfied. The proof of 3.2 is similar to that of Theorem 3.1. By replacing $\Sigma$ by $\Sigma_{T}$ and replacing Assumption 3-Assumption 6 by the results in Lemma 4, the results in Theorem 3.2 follows.

\section{A.4. Proofs for classification using PMLE-LDA}

Lemma 5. Let $\hat{\boldsymbol{\theta}}$ be the estimate of $\boldsymbol{\theta}_{0}$ and $\left\|\hat{\boldsymbol{\theta}}-\hat{\boldsymbol{\theta}}_{0}\right\|_{2}=O_{p}\left(\frac{1}{\sqrt{n p}}\right)$. Define

$$
\tilde{\Sigma}=\Sigma_{T}(\hat{\boldsymbol{\theta}})=\Sigma(\hat{\boldsymbol{\theta}}) \circ K(w)
$$

where $K(w)$ is defined in Section 3.1.2. Assume Assumption 1, Assumption 2 and Assumption 11 and Assumption 12 hold,then

$$
\|\tilde{\Sigma}-\Sigma\|_{2}=O_{p}\left(c_{n}\right) \text { and }\left\|\tilde{\Sigma}^{-1}-\Sigma^{-1}\right\|_{2}=O_{p}\left(c_{n}\right)
$$

where $c_{n, p}=\max \left(\frac{w^{d}}{\sqrt{n p}}, \frac{1}{w}\right)$

Proof.

$$
\begin{aligned}
\|\tilde{\Sigma}-\Sigma\|_{2} & =\left\|\Sigma(\hat{\boldsymbol{\theta}}) \circ K(w)-\Sigma\left(\boldsymbol{\theta}_{0}\right)\right\|_{2} \\
& \leq \max _{i} \sum_{j=1}^{p}\left|r\left(\hat{\boldsymbol{\theta}} ; h_{i j}\right) K_{T}\left(h_{i j}, w\right)-r\left(\boldsymbol{\theta}_{0} ; h_{i j}\right)\right|
\end{aligned}
$$

where $K_{T}(h, w)=\left[(1-h / w)_{+}\right]^{2}$. For any $i=1,2, \ldots, p$,

$$
\begin{aligned}
& \sum_{j=1}^{p}\left|r\left(\hat{\boldsymbol{\theta}} ; h_{i j}\right) K_{T}\left(h_{i j}, w\right)-r\left(\boldsymbol{\theta}_{0} ; h_{i j}\right)\right| \\
\leq & \sum_{h_{i j}<w}\left|r\left(\hat{\boldsymbol{\theta}} ; h_{i j}\right) K_{T}\left(h_{i j}, w\right)-r\left(\boldsymbol{\theta}_{0} ; h_{i j}\right)\right|+\sum_{h_{i j} \geq w}\left|r\left(\boldsymbol{\theta}_{0}, h_{i j}\right)\right|
\end{aligned}
$$




$$
\begin{aligned}
\leq & \sum_{h_{i j}<w}\left|\left(r\left(\hat{\boldsymbol{\theta}} ; h_{i j}\right)-r\left(\boldsymbol{\theta}_{0} ; h_{i j}\right)\right) K_{T}\left(h_{i j}, w\right)\right| \\
& +\sum_{h_{i j}<w}\left|r\left(\theta_{0} ; h_{i j}\right) K_{T}\left(h_{i j}, w\right)-r\left(\boldsymbol{\theta}_{0} ; h_{i j}\right)\right|+\sum_{h_{i j} \geq w}\left|r\left(\boldsymbol{\theta}_{0}, h_{i j}\right)\right| \\
= & (I)+(I I)+(I I I)
\end{aligned}
$$

From the same proof procedure of Lemma 3, we have $(I I)=O_{p}(1 / w)$ and $(I I I)=O_{p}(1 / w)$. From Assumption 1 and Assumption 2(iii), we have

$$
\begin{aligned}
(I) & \leq \sum_{h_{i j}<w}\left|r\left(\hat{\boldsymbol{\theta}} ; h_{i j}\right)-r\left(\boldsymbol{\theta}_{0} ; h_{i j}\right)\right| \leq \sum_{k=1}^{q} \sum_{h_{i j}<w}\left|r_{k}\left(\boldsymbol{\theta}^{*} ; h_{i j}\right)\right|\left|\hat{\theta}_{k}^{*}-\theta_{0 k}\right| \\
& \leq M w^{d} \rho\left\|\hat{\boldsymbol{\theta}}-\boldsymbol{\theta}_{0}\right\|_{2}
\end{aligned}
$$

Therefore $(I)=O_{p}\left(\frac{w^{d}}{\sqrt{n p}}\right)$. Combine (I), (II) and (III), $\|\tilde{\Sigma}-\Sigma\|_{2}=O_{p}\left(c_{n}\right)$.

Since $\Sigma\left(\boldsymbol{\theta}_{0}\right)$ and $\tilde{\Sigma}=\Sigma_{T}(\hat{\boldsymbol{\theta}})$ are positive definite, $\left\|\Sigma^{-1}\right\|_{2}=\frac{1}{\lambda_{\min }(\Sigma)}<\infty$ and $\left\|\tilde{\Sigma}_{T}^{-1}\right\|_{2}=\frac{1}{\lambda_{\min }\left(\tilde{\Sigma}_{T}\right)}<\infty$.

$\left\|\tilde{\Sigma}_{T}^{-1}-\Sigma^{-1}\right\|_{2}=\left\|\Sigma^{-1}\left(\Sigma-\tilde{\Sigma}_{T}\right) \tilde{\Sigma}_{T}^{-1}\right\|_{2} \leq\left\|\Sigma^{-1}\right\|_{2}\left\|\Sigma-\tilde{\Sigma}_{T}\right\|_{2}\left\|\tilde{\Sigma}_{T}^{-1}\right\|_{2}=O_{p}\left(c_{n}\right)$,

and this completes the proof.

Lemma 6. Assume Assumption 12 holds. Then $\max _{1 \leq i \leq s} \sum_{k=s+1}^{p} \sigma_{i k}^{2}$ is bounded above by a constant.

Proof. Since Assumption 12 holds, by similar argument as providing Lemma 3, we have:

$$
\begin{aligned}
\max _{1 \leq i \leq s} \sum_{k=s+1}^{p} \sigma_{i k}^{2} & =\max _{1 \leq i \leq s} \sum_{k=s+1}^{p} \gamma\left(h_{i k}, \boldsymbol{\theta}_{0}\right) \\
\leq & \max _{1 \leq i \leq s}\left(\sum_{0<h_{i j}<1} \gamma\left(h_{i k}, \boldsymbol{\theta}_{0}\right)+\sum_{h_{i j} \geq 1} \gamma\left(h_{i k}, \boldsymbol{\theta}_{0}\right)\right) \\
& \leq \int_{0}^{1} h^{d-1} \gamma\left(h, \boldsymbol{\theta}_{0}\right) d h+\int_{1}^{\infty} h^{d-1} \gamma\left(h, \boldsymbol{\theta}_{0}\right) \\
& \leq \int_{h=0}^{1} h^{d-1} \gamma_{0}\left(h, \boldsymbol{\theta}_{0}\right) d h+\int_{1}^{\infty} h^{d} \gamma_{0}\left(h, \boldsymbol{\theta}_{0}\right) \leq \infty
\end{aligned}
$$

Proof of Theorem 3.3. Suppose a new observation is from class 1, the conditional misclassification rate of $\hat{\delta}_{P M L E}$ for class 1 is:

$$
W_{1}\left(\hat{\delta}_{P M L E}\right)=1-\Phi\left(\frac{\left(\boldsymbol{\mu}_{1}-\overline{\boldsymbol{Y}}-\frac{n_{1}-n_{2}}{2 n} \hat{\boldsymbol{\Delta}}\right)^{T} \tilde{\Sigma}^{-1} \hat{\boldsymbol{\Delta}}}{\sqrt{\hat{\boldsymbol{\Delta}}^{T} \tilde{\Sigma}^{-1} \Sigma \tilde{\Sigma}^{-1} \hat{\boldsymbol{\Delta}}}}\right)
$$


Where $\overline{\boldsymbol{Y}}=\sum_{k=1}^{2} \sum_{i=1}^{n_{k}} \boldsymbol{Y}_{k i}$. We first consider denominator. From Lemma 5, $\|\Sigma-\tilde{\Sigma}\|_{2}=O_{p}\left(c_{n}\right)$ and $\left\|\Sigma^{-1}-\tilde{\Sigma}^{-1}\right\|_{2}=O_{p}\left(c_{n}\right)$, where $c_{n}=\max \left(\frac{w^{d}}{\sqrt{n p}}, \frac{1}{w}\right)$ and $w$ is the threshold distance $w$. Then

$$
\begin{aligned}
\hat{\boldsymbol{\Delta}}^{T} \tilde{\Sigma}^{-1} \Sigma \tilde{\Sigma}^{-1} \hat{\boldsymbol{\Delta}} & =\hat{\boldsymbol{\Delta}}^{T} \tilde{\Sigma}^{-1} \hat{\boldsymbol{\Delta}}+\hat{\boldsymbol{\Delta}}^{T} \tilde{\Sigma}^{-1}(\Sigma-\tilde{\Sigma}) \tilde{\Sigma}^{-1} \hat{\boldsymbol{\Delta}} \\
& \leq \hat{\boldsymbol{\Delta}}^{T} \tilde{\Sigma}^{-1} \hat{\boldsymbol{\Delta}}+\frac{\|\Sigma-\tilde{\Sigma}\|_{2}}{\lambda_{\min }(\tilde{\Sigma})} \hat{\boldsymbol{\Delta}}^{T} \tilde{\Sigma}^{-1} \hat{\boldsymbol{\Delta}} \\
& =\hat{\boldsymbol{\Delta}}^{T} \tilde{\Sigma}^{-1} \hat{\boldsymbol{\Delta}}\left(1+O_{p}\left(c_{n}\right)\right) \\
& =\left(\hat{\boldsymbol{\Delta}}^{T} \Sigma^{-1} \hat{\boldsymbol{\Delta}}+\hat{\boldsymbol{\Delta}}^{T}\left(\tilde{\Sigma}^{-1}-\Sigma^{-1}\right) \hat{\boldsymbol{\Delta}}\right)\left(1+O_{p}\left(c_{n}\right)\right) \\
& =\hat{\boldsymbol{\Delta}}^{T} \Sigma^{-1} \hat{\boldsymbol{\Delta}}\left(1+O_{p}\left(c_{n}\right)\right)
\end{aligned}
$$

Write

$$
\hat{\boldsymbol{\Delta}}^{T} \Sigma^{-1} \hat{\boldsymbol{\Delta}}=\boldsymbol{\Delta} \Sigma^{-1} \boldsymbol{\Delta}+2(\hat{\boldsymbol{\Delta}}-\boldsymbol{\Delta})^{T} \Sigma^{-1} \boldsymbol{\Delta}+(\hat{\boldsymbol{\Delta}}-\boldsymbol{\Delta})^{T} \Sigma^{-1}(\hat{\boldsymbol{\Delta}}-\boldsymbol{\Delta})
$$

From Theorem 3.1, $\|\hat{\boldsymbol{\Delta}}-\boldsymbol{\Delta}\|_{2}=O_{P}\left(\sqrt{\frac{s}{n}}\right)$. Hence $(\hat{\boldsymbol{\Delta}}-\boldsymbol{\Delta})^{T} \Sigma^{-1}(\hat{\boldsymbol{\Delta}}-\boldsymbol{\Delta})=$ $O_{P}\left(\frac{s}{n}\right)$. Also the second term

$$
(\hat{\boldsymbol{\Delta}}-\boldsymbol{\Delta})^{T} \Sigma^{-1} \boldsymbol{\Delta} \leq\left(\boldsymbol{\Delta}^{T} \Sigma^{-1} \boldsymbol{\Delta}\right)^{\frac{1}{2}}\left((\hat{\boldsymbol{\Delta}}-\boldsymbol{\Delta})^{T} \Sigma^{-1}(\hat{\boldsymbol{\Delta}}-\boldsymbol{\Delta})\right)^{\frac{1}{2}}
$$

Since $\frac{s}{n \Delta^{T} \Sigma^{-1} \boldsymbol{\Delta}} \rightarrow 0$, we have

$$
\begin{aligned}
\hat{\boldsymbol{\Delta}}^{T} \Sigma^{-1} \hat{\boldsymbol{\Delta}}= & \boldsymbol{\Delta}^{T} \Sigma^{-1} \boldsymbol{\Delta}\left(1+O_{P}\left(\sqrt{\frac{s}{n \boldsymbol{\Delta}^{T} \Sigma^{-1} \boldsymbol{\Delta}}}\right)+O_{P}\left(\frac{s}{n \boldsymbol{\Delta}^{T} \Sigma^{-1} \boldsymbol{\Delta}}\right)\right) \\
& =\boldsymbol{\Delta}^{T} \Sigma^{-1} \boldsymbol{\Delta}\left(1+O_{P}\left(\sqrt{\frac{s}{n \boldsymbol{\Delta}^{T} \Sigma^{-1} \boldsymbol{\Delta}}}\right)\right)
\end{aligned}
$$

Let $D_{n, p}=\max \left(\sqrt{\frac{s}{n \Delta^{T} \Sigma^{-1} \boldsymbol{\Delta}}}, c_{n}\right)$, the denominator can be represented by: $\sqrt{\hat{\boldsymbol{\Delta}}^{T} \tilde{\Sigma}^{-1} \Sigma \tilde{\Sigma}^{-1} \hat{\boldsymbol{\Delta}}}=\sqrt{\boldsymbol{\Delta} \Sigma^{-1} \boldsymbol{\Delta}\left(1+O_{p}\left(D_{n, p}\right)\right)}$.

Now consider the nominator.

$$
\begin{aligned}
& \left(\boldsymbol{\mu}_{1}-\overline{\boldsymbol{Y}}-\frac{n_{1}-n_{2}}{2 n} \hat{\boldsymbol{\Delta}}\right)^{T} \tilde{\Sigma}^{-1} \hat{\boldsymbol{\Delta}} \\
& =\left(\boldsymbol{\mu}_{1}-\overline{\boldsymbol{Y}}\right)^{T} \tilde{\Sigma}^{-1} \hat{\boldsymbol{\Delta}}+\frac{n_{2}-n_{1}}{2 n} \hat{\boldsymbol{\Delta}}^{T} \tilde{\Sigma}^{-1} \hat{\boldsymbol{\Delta}} \\
& =\left(\boldsymbol{\mu}_{1}-\overline{\boldsymbol{Y}}-\frac{n_{2}}{n} \boldsymbol{\Delta}\right)^{T} \tilde{\Sigma}^{-1} \hat{\boldsymbol{\Delta}}+\frac{n_{2}}{n} \boldsymbol{\Delta}^{T} \tilde{\Sigma}^{-1} \hat{\boldsymbol{\Delta}}+\frac{n_{2}-n_{1}}{2 n} \hat{\boldsymbol{\Delta}}^{T} \tilde{\Sigma}^{-1} \hat{\boldsymbol{\Delta}} \\
& =(1)+(2)+(3)
\end{aligned}
$$

We start from (3). By Lemma $5, \hat{\boldsymbol{\Delta}}^{T} \tilde{\Sigma}^{-1} \hat{\boldsymbol{\Delta}}=\hat{\boldsymbol{\Delta}}^{T} \Sigma^{-1} \hat{\boldsymbol{\Delta}}\left(1+O_{p}\left(c_{n}\right)\right)$. From (A.61), (3) can be represented by (3) $=\frac{n_{2}-n_{1}}{2 n} \boldsymbol{\Delta}^{T} \Sigma^{-1} \boldsymbol{\Delta}\left(1+O_{P}\left(D_{n, p}\right)\right)$. 
For (2), first we have $\boldsymbol{\Delta}^{T} \tilde{\Sigma}^{-1} \hat{\boldsymbol{\Delta}}=\boldsymbol{\Delta}^{T} \Sigma^{-1} \hat{\boldsymbol{\Delta}}\left(1+O_{p}\left(c_{n}\right)\right)$. Then combine (A.61)

$$
\boldsymbol{\Delta}^{T} \Sigma^{-1} \hat{\boldsymbol{\Delta}} \leq\left(\boldsymbol{\Delta}^{T} \Sigma^{-1} \boldsymbol{\Delta}\right)^{\frac{1}{2}}\left(\hat{\boldsymbol{\Delta}}^{T} \Sigma^{-1} \hat{\boldsymbol{\Delta}}\right)^{\frac{1}{2}}=\boldsymbol{\Delta}^{T} \Sigma^{-1} \boldsymbol{\Delta}\left(1+O_{P}\left(\sqrt{\frac{s}{n \boldsymbol{\Delta}^{T} \Sigma^{-1} \boldsymbol{\Delta}}}\right)\right)
$$

Then (2) can be represented by: (2) $=\frac{n_{2}}{n} \boldsymbol{\Delta}^{T} \Sigma^{-1} \hat{\boldsymbol{\Delta}}=\frac{n_{2}}{n} \boldsymbol{\Delta}^{T} \Sigma^{-1} \boldsymbol{\Delta}(1+$ $\left.O_{P}\left(D_{n, p}\right)\right)$.

Thus

$$
(2)+(3)=\frac{1}{2} \boldsymbol{\Delta}^{T} \Sigma^{-1} \boldsymbol{\Delta}\left(1+O_{P}\left(D_{n, p}\right)\right)
$$

Now we consider $(1)$. Let $\hat{\boldsymbol{\Delta}}=\left(\hat{\boldsymbol{\Delta}}_{1}^{T}, \hat{\boldsymbol{\Delta}}_{2}^{T}\right)^{T}$ where $\hat{\boldsymbol{\Delta}}_{1}$ is $s$ dimension and $\hat{\boldsymbol{\Delta}}_{2}$ is $p-s$ dimension. From Theorem 3.1, with probability tending to $1, \hat{\boldsymbol{\Delta}}_{2}=0$ and $\|\hat{\boldsymbol{\Delta}}-\boldsymbol{\Delta}\|_{2}=O_{P}\left(\frac{s}{n}\right)$. Let $\xi=\boldsymbol{\mu}_{1}-\overline{\boldsymbol{Y}}-\frac{n_{2}}{n} \boldsymbol{\Delta}=\left(\xi_{1}^{T}, \xi_{0}^{T}\right)^{T}$, where $\xi_{1}$ is $s$ dimension and $\xi_{0}$ is $p-s$ dimension. Then $\xi \sim N\left(0, \frac{1}{n} \Sigma\right)$

Write

$$
\begin{aligned}
& \Sigma=\left(\begin{array}{ll}
\Sigma_{1} & \Sigma_{12} \\
\Sigma_{12}^{T} & \Sigma_{2}
\end{array}\right), \quad \Sigma^{-1}=\left(\begin{array}{ll}
C_{1} & C_{12} \\
C_{12}^{T} & C_{2}
\end{array}\right) \\
& \tilde{\Sigma}=\left(\begin{array}{ll}
\tilde{\Sigma}_{1} & \tilde{\Sigma}_{12} \\
\tilde{\Sigma}_{12}^{T} & \tilde{\Sigma}_{2}
\end{array}\right), \quad \tilde{\Sigma}^{-1}=\left(\begin{array}{ll}
\tilde{C}_{1} & \tilde{C}_{12} \\
\tilde{C}_{12}^{T} & \tilde{C}_{2}
\end{array}\right)
\end{aligned}
$$

where $\Sigma_{1}, \tilde{\Sigma}_{1}, C_{1}$ and $\tilde{C}_{1}$ are $s \times s$ matrix. Then

$$
C_{12}=-\Sigma_{1}^{-1} \Sigma_{12} C_{2} \text { and } \tilde{C}_{12}=-\tilde{\Sigma}_{1}^{-1} \tilde{\Sigma}_{12} \tilde{C}_{2} .
$$

Write

$$
\begin{aligned}
(1) & =\xi^{T} \tilde{\Sigma}^{-1} \hat{\boldsymbol{\Delta}}=\xi_{1}^{T} \tilde{C}_{1} \hat{\boldsymbol{\Delta}}_{1}+\xi_{0}^{T} \tilde{C}_{12} \hat{\boldsymbol{\Delta}}_{1} \\
& =\xi_{1}^{T} \tilde{C}_{1} \hat{\boldsymbol{\Delta}}_{1}-\xi_{0} \tilde{C}_{2} \tilde{\Sigma}_{2} \tilde{\Sigma}_{1}^{-1} \hat{\boldsymbol{\Delta}}_{1} \\
& =(i)+(i i)
\end{aligned}
$$

First we have: $\xi_{1}^{T} \tilde{C}_{1} \hat{\boldsymbol{\Delta}}_{1} \leq\left(\xi_{1}^{T} \tilde{C}_{1} \xi_{1}\right)^{1 / 2}\left(\hat{\boldsymbol{\Delta}}_{1}^{T} \tilde{C}_{1} \hat{\boldsymbol{\Delta}}_{1}\right)^{1 / 2}$. By Lemma 5 ,

$$
\xi_{1}^{T} \tilde{C}_{1} \xi_{1}=\xi_{1}^{T} C_{1} \xi_{1}\left(1+O_{p}\left(c_{n}\right)\right)
$$

Since $\xi_{1} \sim N\left(0, \frac{1}{n} \Sigma_{1}\right), E\left(\xi_{1}^{T} \Sigma_{1}^{-1} \xi_{1}\right)=\operatorname{tr}\left(\frac{1}{n} I_{s}\right)=\frac{s}{n}$. Then $\xi_{1}^{T} \Sigma_{1}^{-1} \xi_{1}=O_{P}\left(\frac{s}{n}\right)$ and therefore $\xi_{1}^{T} C_{1} \xi_{1}^{T} \leq \xi_{1}^{T} \Sigma^{-1} \xi_{1}^{T} \frac{\lambda_{\max }\left(C_{1}\right)}{\lambda_{\min }\left(\Sigma_{1}^{-1}\right)}=O_{p}\left(\sqrt{\frac{s}{n}}\right)$. Hence $\xi_{1}^{T} \tilde{C}_{1} \xi_{1}=O_{P}\left(\frac{s}{n}\right)$.

Also,

$$
\hat{\boldsymbol{\Delta}}_{1}^{T} \tilde{C}_{1} \hat{\boldsymbol{\Delta}}_{1}=\hat{\boldsymbol{\Delta}}^{T} \tilde{\Sigma}^{-1} \hat{\boldsymbol{\Delta}}=\boldsymbol{\Delta}^{T} \Sigma^{-1} \boldsymbol{\Delta}\left(1+O_{p}\left(D_{n, p}\right)\right)
$$

Hence $(i)$ in $(1)$ is: $(i)=\xi_{1}^{T} \tilde{C}_{1} \hat{\boldsymbol{\Delta}}_{1}=\left(\boldsymbol{\Delta}^{T} \Sigma^{-1} \boldsymbol{\Delta}\right)^{\frac{1}{2}} O_{p}\left(\sqrt{\frac{s}{n}}\right)$. 
The second term in (1) is:

$$
(i i)=\xi_{0} \tilde{C}_{2} \tilde{\Sigma}_{12} \tilde{\Sigma}_{1}^{-1} \hat{\boldsymbol{\Delta}}_{1} \leq\left(\hat{\boldsymbol{\Delta}}_{1} \tilde{\Sigma}_{1}^{-1} \hat{\boldsymbol{\Delta}}_{1}\right)^{1 / 2}\left(\xi_{0} \tilde{C}_{2}^{T} \tilde{\Sigma}_{12}^{T} \tilde{\Sigma}_{1}^{-1} \tilde{\Sigma}_{12} \tilde{C}_{2}\right)^{1 / 2}
$$

By Lemma 5,

$$
\xi_{0}^{T} \tilde{C}_{2}^{T} \tilde{\Sigma}_{12}^{T} \tilde{\Sigma}_{1}^{-1} \tilde{\Sigma}_{12} \tilde{C}_{2} \xi_{0}=\xi_{0} C_{2}^{T} \Sigma_{12}^{T} \Sigma_{1}^{-1} \Sigma_{12} C_{2} \xi_{0}\left(1+O_{p}\left(c_{n}\right)\right)
$$

Since $\xi_{0} \sim N\left(0, \frac{1}{n} \Sigma_{2}\right)$,

$$
\begin{aligned}
E\left[\xi_{0}^{T} C_{2}^{T} \Sigma_{12}^{T} \Sigma_{1}^{-1} \Sigma_{12} C_{2} \xi_{0}\right] & \leq \lambda_{\max }\left(\Sigma_{1}^{-1}\right) E\left[\xi_{0}^{T} C_{2}^{T} \Sigma_{12}^{T} \Sigma_{12} C_{2} \xi_{0}\right] \\
& =\lambda_{\max }\left(\Sigma_{1}^{-1}\right) \operatorname{tr}\left(E\left[\xi_{0}^{T} C_{2}^{T} \Sigma_{12}^{T} \Sigma_{12} C_{2} \xi_{0}\right]\right) \\
& =\lambda_{\max }\left(\Sigma_{1}^{-1}\right) \frac{1}{n} \operatorname{tr}\left(C_{2}^{T} \Sigma_{12}^{T} \Sigma_{12} C_{2} \Sigma_{2}\right) \\
& \leq \lambda_{\max }\left(\Sigma_{1}^{-1}\right) \lambda_{\max }\left(\Sigma_{2}\right) \lambda_{\max }^{2}\left(C_{2}\right) \frac{1}{n} \operatorname{tr}\left(\Sigma_{12} \Sigma_{12}^{T}\right) . \\
\operatorname{tr}\left(\Sigma_{12} \Sigma_{12}^{T}\right) & =\sum_{i=1}^{s} \sum_{k=s+1}^{p} \sigma_{i k}^{2} \leq s \max _{1 \leq i \leq s} \sum_{k=s+1}^{p} \sigma_{i k}^{2},
\end{aligned}
$$

thus $\xi_{0}^{T} C_{2}^{T} \Sigma_{12}^{T} \Sigma_{1}^{-1} \Sigma_{12} C_{2} \xi_{0}=O_{p}\left(\frac{s}{n} d_{n, p}\right)$, where $d_{n, p}=\max _{1 \leq i \leq s} \sum_{k=s+1}^{p} \sigma_{i k}^{2}$. By Lemma $6, d_{n, p}$ is bounded above by a constant. As a result:

$$
\begin{aligned}
\xi_{0}^{T} \tilde{C}_{2}^{T} \tilde{\Sigma}_{12}^{T} \tilde{\Sigma}_{1}^{-1} \tilde{\Sigma}_{12} \tilde{C}_{2} \xi_{0} & =x i_{0}^{T} C_{2}^{T} \Sigma_{12}^{T} \Sigma_{1}^{-1} \Sigma_{12} C_{2} \xi_{0} \\
& =O_{p}\left(\frac{s}{n} d_{n, p}\right)\left(1+O_{p}\left(\max \left(c_{n}, \sqrt{\frac{s}{n}}\right)\right)\right)
\end{aligned}
$$

Since

$$
\hat{\boldsymbol{\Delta}}_{1}^{T} \tilde{\Sigma}_{1}^{-1} \hat{\boldsymbol{\Delta}}_{1} \leq \hat{\boldsymbol{\Delta}}_{1}^{T} \tilde{C}_{1} \hat{\boldsymbol{\Delta}}_{1} \leq \hat{\boldsymbol{\Delta}}^{T} \tilde{\Sigma}^{-1} \hat{\boldsymbol{\Delta}}=\boldsymbol{\Delta}^{T} \Sigma^{-1} \boldsymbol{\Delta}\left(1+O_{P}\left(D_{n, p}\right)\right)
$$

Let $A_{n, p}=\max \left(\sqrt{\frac{s}{n \boldsymbol{\Delta}^{T} \Sigma^{-1} \boldsymbol{\Delta}}}, \frac{s}{n}, c_{n}\right)$, we have

$$
\left.(\text { ii })=\left(\boldsymbol{\Delta}^{T} \Sigma^{-1} \boldsymbol{\Delta}\right)^{\frac{1}{2}} O_{P}\left(A_{n, p}\right)\right)
$$

Combining the approximation of $(i)$ and $(i i)$ in (1), we have (1) $=\xi^{T} \tilde{\Sigma}^{-1} \hat{\boldsymbol{\Delta}}=$ $\left(\boldsymbol{\Delta}^{T} \Sigma^{-1} \boldsymbol{\Delta}\right)^{\frac{1}{2}} O_{P}\left(A_{n, p}\right)$.

As a result, since $\frac{\sqrt{s / n}}{C_{p}} \rightarrow 0$,

$$
\begin{aligned}
W_{1}\left(\hat{\delta}_{P L D A}\right) & =1-\Phi\left(\frac{\frac{1}{2} \boldsymbol{\Delta}^{T} \Sigma^{-1} \boldsymbol{\Delta}\left(1+O_{p}\left(D_{n, p}\right)\right)+\sqrt{\boldsymbol{\Delta}^{T} \Sigma^{-1} \boldsymbol{\Delta}} O_{p}\left(A_{n, p}\right)}{\sqrt{\boldsymbol{\Delta}^{T} \Sigma^{-1} \boldsymbol{\Delta}\left(1+O_{p}\left(D_{n, p}\right)\right)}}\right) \\
& =1-\Phi\left(\frac{\frac{1}{2} C_{p}\left(1+O_{p}\left(D_{n, p}\right)+\sqrt{C_{p}} O_{p}\left(A_{n, p}\right)\right.}{\sqrt{C_{p}}\left(1+O_{p}\left(D_{n, p}\right)\right)}\right)
\end{aligned}
$$


Similarly, we can derive:

$$
\begin{aligned}
W_{2}\left(\hat{\delta}_{P L D A}\right) & =\Phi\left(\frac{-\frac{1}{2} \boldsymbol{\Delta}^{T} \Sigma^{-1} \boldsymbol{\Delta}\left(1+O_{p}\left(D_{n, p}\right)\right)+\sqrt{\boldsymbol{\Delta}^{T} \Sigma^{-1} \boldsymbol{\Delta}} O_{p}\left(A_{n, p}\right)}{\sqrt{\boldsymbol{\Delta}^{T} \Sigma^{-1} \boldsymbol{\Delta}\left(1+O_{p}\left(D_{n, p}\right)\right)}}\right) \\
& =\Phi\left(\frac{-\frac{1}{2} C_{p}\left(1+O_{p}\left(D_{n, p}\right)+\sqrt{C_{p}} O_{p}\left(A_{n, p}\right)\right.}{\sqrt{C_{p}}\left(1+O_{p}\left(D_{n, p}\right)\right)}\right)
\end{aligned}
$$

Since $D_{n, p} \rightarrow 0$ and $A_{n, p} \rightarrow 0$, both $W_{1}\left(\hat{\delta}_{P L D A}\right)$ and $W_{2}\left(\hat{\delta}_{P L D A}\right)$ go to $1-$ $\Phi\left(\frac{\sqrt{C_{0}}}{2}\right)$ as $n, p \rightarrow \infty$ with probability tending to 1 as $n, p, s \rightarrow \infty$. Then the approximate overall misclassification error rate is $W\left(\hat{\delta}_{P L D A}\right)=\frac{1}{2}\left(W_{1}\left(\hat{\delta}_{P L D A}\right)+\right.$ $\left.W_{2}\left(\hat{\delta}_{P L D A}\right)\right) \rightarrow 1-\Phi\left(\frac{\sqrt{C_{0}}}{2}\right)$. This completes the proof of sub-optimal.

Now we show the asymptotically optimal of $W\left(\hat{\delta}_{P L D A}\right)$. If $C_{p} \rightarrow C_{0}<\infty$, $\frac{W\left(\hat{\delta}_{P L D A}\right)}{W_{O P T}}=\frac{W\left(\hat{\delta}_{P L D A}\right)}{\Phi\left(-\frac{\sqrt{C_{p}}}{2}\right)} \rightarrow 1$.

If $C_{p} \rightarrow \infty$,

$$
\frac{x \sqrt{C_{p}}}{4+x^{2}} e^{-\frac{x^{2}-C_{p}}{8}} \leq \frac{W\left(\hat{\delta}_{P L D A}\right)}{\Phi\left(-\frac{\sqrt{C_{p}}}{2}\right)} \leq \frac{4+C_{p}}{x \sqrt{C_{p}}} e^{-\frac{x^{2}-C_{p}}{8}}
$$

where $x=\frac{C_{p}\left(1+O_{p}\left(D_{n, p}\right)\right) \pm 2 \sqrt{C_{p}} O_{p}\left(A_{n, p}\right)}{\sqrt{\overline{C_{p}}\left(1+O_{p}\left(D_{n, p}\right)\right)}}=\sqrt{C_{p}}\left(1+O_{p}\left(D_{n, p} \pm O\left(\frac{A_{n, p}}{\sqrt{C_{p}}}\right)\right)\right)$.

First $\frac{x \sqrt{C_{p}}}{4+x^{2}} e^{-\frac{x^{2}-C_{p}}{8}} \rightarrow 1$ and $\frac{4+C_{p}}{x \sqrt{C_{p}}} \rightarrow 1$ as $C_{p} \rightarrow \infty$. Also,

$$
x^{2}-C_{p}=C_{p}\left(O\left(D_{n, p}+O\left(\frac{A_{n, p}}{\sqrt{C_{p}}}\right)\right)\right)
$$

if $C_{p} c_{n} \rightarrow 0$ and $C_{p} \sqrt{\frac{s}{n}} \rightarrow 0$, we have $x^{2}-C_{p} \rightarrow 0$. Hence $\frac{W\left(\hat{\delta}_{P L D A}\right)}{W_{O P T}} \rightarrow 1$. This completes the proof.

\section{Acknowledgements}

The research is partially supported by NSF-DMS 1945824 .

\section{References}

[1] BACHOC, F. and FurRer, R. (2016). On the smallest eigenvalues of covariance matrices of multivariate spatial processes. Stat 5 102-107. MR3478800

[2] Bhattacharya, A., Chakraborty, A. and Mallick, B. K. (2016). Fast sampling with Gaussian scale mixture priors in high-dimensional regression. Biometrika 103 985-991. MR3620452

[3] Bickel, P. J. and Levina, E. (2004). Some theory for Fisher's linear discriminant function, 'naive Bayes', and some alternatives when there are many more variables than observations. Bernoulli 10 989-1010. MR2108040 
[4] Bickel, P. J. and Levina, E. (2008a). Covariance regularization by thresholding. The Annals of Statistics 36 2577-2604. MR2485008

[5] Bickel, P. J. and LevinA, E. (2008b). Regularized estimation of large covariance matrices. The Annals of Statistics 36 199-227. MR2387969

[6] Bowman, F. D. (2014). Brain Imaging Analysis. Annu. Rev. Appl. 1 6185.

[7] CAI, T. T. and LiU, W. (2011). A direct estimation approach to sparse linear discriminant analysis. Journal of the American Statistical Association 106 1566-1577. MR2896857

[8] CAI, T. T. and Zhang, A. (2016). Minimax rate-optimal estimation of high-dimensional covariance matrices with incomplete data. Journal of Multivariate Analysis 150 55-74. MR3534902

[9] CAI, T. T. and ZhAng, L. (2018). High-dimensional Linear Discriminant Analysis: Optimality, Adaptive Algorithm, and Missing Data1. Technical report. MR3997097

[10] Cai, T. T., Ren, Z., Zhou, H. H. et al. (2016). Estimating structured high-dimensional covariance and precision matrices: Optimal rates and adaptive estimation. Electronic Journal of Statistics 10 1-59. MR3466172

[11] Cannings, T. I. and Samworth, R. J. (2017). Random-projection ensemble classification. Journal of the Royal Statistical Society: Series B (Statistical Methodology) 79 959-1035. MR3689307

[12] Chu, T., Zhu, J., Wang, H. et al. (2011). Penalized maximum likelihood estimation and variable selection in geostatistics. The Annals of Statistics 39 2607-2625. MR2906880

[13] Cox, R. W. (1996). AFNI: software for analysis and visualization of functional magnetic resonance neuroimages. Computers and Biomedical Research 29 162-173.

[14] Cressie, N. (1992). Statistics for spatial data. Terra Nova 4 613-617. MR1127423

[15] FAn, J. and FAn, Y. (2008). High dimensional classification using features annealed independence rules. Annals of Statistics 36 2605. MR2485009

[16] FAn, J., Feng, Y. and Tong, X. (2012). A road to classification in high dimensional space: the regularized optimal affine discriminant. Journal of the Royal Statistical Society: Series B (Statistical Methodology) 74 745-771. MR2965958

[17] FAN, J. and LI, R. (2001). Variable selection via nonconcave penalized likelihood and its oracle properties. Journal of the American Statistical Association 96 1348-1360. MR1946581

[18] Feng, W., Sarkar, A., Lim, C. Y. and Maiti, T. (2016). Variable selection for binary spatial regression: Penalized quasi-likelihood approach. Biometrics 72 1164-1172. MR3591601

[19] Friedman, J., Hastie, T. and Tibshirani, R. (2001). The Elements of Statistical Learning 1. Springer Series in Statistics. New York, NY, USA. MR2722294

[20] Fu, R., Thurman, A. L., Chu, T., Steen-Adams, M. M. and Zhu, J. (2013). On estimation and selection of autologistic regression models via 
penalized pseudolikelihood. Journal of Agricultural, Biological, and Environmental Statistics 18 429-449. MR3110901

[21] Hoeting, J. A., Davis, R. A., Merton, A. A. and Thompson, S. E. (2006). Model selection for geostatistical models. Ecological Applications 16 87-98.

[22] Horn, R. and Johnson, C. (1992). Topics in matrix analysis. ZAMMJournal of Applied Mathematics and Mechanics 72 692-692. MR1091716

[23] Huang, H.-C. and Chen, C.-S. (2007). Optimal geostatistical model selection. Journal of the American Statistical Association 102 1009-1024. MR2411661

[24] Jack, C. R., Bernstein, M. A., Fox, N. C., Thompson, P., AlexanDer, G., Harvey, D., Borowski, B., Britson, P. J., L Whitwell, J., WARD, C. et al. (2008). The Alzheimer's disease neuroimaging initiative (ADNI): MRI methods. Journal of Magnetic Resonance Imaging 27 685691.

[25] LI, Y. (2018). High Dimensional Classification for Spatially Dependent Data with Application to Neuroimaging. Michigan State University.

[26] Li, Q. and Shao, J. (2015). Sparse quadratic discriminant analysis for high dimensional data. Statistica Sinica 457-473. MR3379082

[27] Li, L. and Zhang, X. (2017). Parsimonious tensor response regression. Journal of the American Statistical Association 112 1131-1146. MR3735365

[28] Li, Y., Zhang, L., Bozoki, A., Zhu, D. C., Choi, J. and Maiti, T. (2019). Early prediction of Alzheimer's disease using longitudinal volumetric MRI data from ADNI. Health Services and Outcomes Research Methodology 1-27.

[29] Lindquist, M. A. (2008). The statistical analysis of fMRI data. Statistical Science 23 439-464. MR2530545

[30] MaI, Q., Zou, H. and YuAN, M. (2012). A direct approach to sparse discriminant analysis in ultra-high dimensions. Biometrika asr066. MR2899661

[31] Mardia, K. V. and Marshall, R. J. (1984). Maximum likelihood estimation of models for residual covariance in spatial regression. Biometrika 71 135-146. MR0738334

[32] Meyer, C. D. (2001). Matrix Analysis and Applied Linear Algebra. SIAM, Philadelphia. MR1777382

[33] Musgrove, D. R., Hughes, J. and Eberly, L. E. (2016). Fast, fully Bayesian spatiotemporal inference for fMRI data. Biostatistics 17 291-303. MR3516001

[34] Nandy, S., Lim, C. Y. and Maiti, T. (2017). Additive model building for spatial regression. Journal of the Royal Statistical Society: Series B (Statistical Methodology) 79 779-800. MR3641407

[35] PAN, Y., MAI, Q. and ZhANG, X. (2019). Covariate-adjusted tensor classification in high dimensions. Journal of the American Statistical Association 114 1305-1319. MR4011781

[36] Reyes, P. E., Zhu, J. and Aukema, B. H. (2012). Selection of spatial- 
temporal lattice models: assessing the impact of climate conditions on a mountain pine beetle outbreak. Journal of Agricultural, Biological, and Environmental Statistics 17 508-525. MR2993279

[37] Rothman, A. J., Bickel, P. J., Levina, E., Zhu, J. et al. (2008). Sparse permutation invariant covariance estimation. Electronic Journal of Statistics 2 494-515. MR2417391

[38] Shao, J., Wang, Y., Deng, X., Wang, S. et al. (2011). Sparse linear discriminant analysis by thresholding for high dimensional data. The Annals of Statistics 39 1241-1265. MR2816353

[39] Smith, M. and Fahrmeir, L. (2007). Spatial Bayesian variable selection with application to functional magnetic resonance imaging. Journal of the American Statistical Association 102 417-431. MR2370843

[40] Tibshirani, R., Hastie, T., Narasimhan, B. and Chu, G. (2002). Diagnosis of multiple cancer types by shrunken centroids of gene expression. Proceedings of the National Academy of Sciences 99 6567-6572.

[41] Wendland, H. (1995). Piecewise polynomial, positive definite and compactly supported radial functions of minimal degree. Advances in Computational Mathematics 4 389-396. MR1366510

[42] Witten, D. M. and Tibshirani, R. (2011). Penalized classification using Fisher's linear discriminant. Journal of the Royal Statistical Society: Series $B$ (Statistical Methodology) 73 753-772. MR2867457

[43] Worsley, K. (2003). Developments in random field theory. Human Brain Function 2 881-886.

[44] Wyman, B. T., Harvey, D. J., Crawford, K., Bernstein, M. A., Carmichael, O., Cole, P. E., Crane, P. K., DeCarli, C., Fox, N. C., Gunter, J. L. et al. (2013). Standardization of analysis sets for reporting results from ADNI MRI data. Alzheimer's 6 Dementia: The Journal of the Alzheimer's Association 9 332-337.

[45] Xu, P., Zhu, J., Zhu, L. and Li, Y. (2014). Covariance-enhanced discriminant analysis. Biometrika 102 33-45. MR3335094

[46] Zhang, L., Lim, C. Y., Maiti, T., Li, Y., Choi, J., Bozoki, A., ZHU, D. C., for the Key Laboratory of Applied Statistics of the Ministry of Education (KLAS), and for the Alzheimer's Disease Neuroimaging Initiative (2019). Analysis of conversion of Alzheimer's disease using a multi-state Markov model. Statistical Methods in Medical Research $\mathbf{2 8}$ 2801-2819. MR4000196

[47] Zhu, J., HuAng, H.-C. and Reyes, P. E. (2010). On selection of spatial linear models for lattice data. Journal of the Royal Statistical Society: Series $B$ (Statistical Methodology) 72 389-402. MR2758118

[48] Zou, H. and Li, R. (2008). One-step sparse estimates in nonconcave penalized likelihood models. Annals of Statistics 36 1509. MR2435443 